NASA Technical Memorandum 88350

\title{
Composition, Structure, and Chemistry of Interstellar Dust
}

A. G. G. M. Tielens, Ames Research Center, Moffett Field, California

L. J. Allamandola, University of California, Berkeley, California 
COMPOSITION, STRUCTURE, AND CHEMISTRY OF INTERSTELLAR DUST

\author{
A. G. G. M. Tielens ${ }^{1,2}$ and L. J. Allamandola ${ }^{1}$ \\ 1'Space Sciences Division, MS 245-6 \\ NASA Ames Research Center \\ Moffett Field, California 94035, U.S.A. \\ ${ }^{2}$ Space Sciences Laboratory \\ University of California \\ Berkeley, California 94720, U.S.A.
}

\title{
INTRODUCTION AND SUMMARY
}

Interstellar dust is an important component of the interstellar medium. It dominates the heating and cooling of clouds through energetic photoelectrons and gas-grain collisions (see the chapter by Black in this volume). It is also the dominant source of opacity and determines, therefore, the spectrum of dust-enshrouded objects. Unquestionably, molecular hydrogen, the most abundant gas-phase species in molecular clouds, is formed on grain surfaces. Other gas-phase molecules might result from reactions on grain surfaces as well. Grains can also influence the gas-phase composition of molecular clouds indirectly, because they may lock up some of the elements. The metals (e.g., iron) are particularly important in this respect since they tend to carry the charge inside a dense cloud and, thus, regulate the ion-molecule chemistry (see the chapter by Prasad in this volume). Such depletions will, of course, also influence the cooling balance.

Yet, despite the importance of the dust and despite 50 years of active research in this field, many of the major questions either remain unanswered or the answers that have been proposed are highly controversial. These include the two basic ones: What is the composition of the interstellar dust? and Where and how is it formed? These questions are of course interrelated and an answer to one suggests an answer to the other.

The dust components proposed to be present in the interstellar medium can be divided into two classes depending on their formation

This work was supported through NASA grant NCA2-1R050-405.

Presented at the summerschool on Interstellar Processes, Jackson, Wyoming, July 1986. 
history. First, there is stardust, such as silicates, graphite and amorphous carbon, which are made in the oxygen-rich or carbon-rich outflow from late-type giants and planetary nebulae and, possibly, novae and supernovae. Dust formation has been theoretically predicted to occur in such outflows (Hoyle and Wickramasinghe 1962; Kamei jo 1963). This was subsequently confirmed, mainly by infrared observations, in the early seventies. There is now abundant evidence for the formation of dust in these high-density, high-temperature environments ( $c$. the reviews by Merrill 1977 and Aitken 1981). Second, there are dust components that are formed in the interstellar medium itself. This includes "icy" grain mantles, consisting of simple molecules (e.g., $\mathrm{H}_{2} \mathrm{O}, \mathrm{NH}_{3}$, $\mathrm{CH}_{3} \mathrm{OH}$, and $\mathrm{CO}$ ) inside dense clouds, as well as an organic refractory dust component, consisting of more complex molecules, in the diffuse interstellar medium. The presence of both of these dust components in the interstellar medium has been revealed by infrared spectroscopy of highly obscured objects. The icy grain mantles, which form by accretion and reaction of gas-phase species on preexisting cores, seem to be ubiquitous inside molecular clouds (Merrill et al. 1976; Joyce and Simon 1982; Willner et al. 1982). In contrast, the organic refractory component has, at present, only been detected toward the galactic center (Willner et al. 1979; Allen and Wickramasinghe 1981; Butchart et al. 1985). Presumably, there is a chemical, evolutionary link between these two dust components. That is, the organic refractory dust component is formed by energetic processing (UV photolysis or cosmic ray bombardment) of the icy grain mantles (Greenberg 1982; Moore and Donn 1982; Strazulla et al. 1983).

Detailed models of interstellar extinction have been constructed based on stardust alone (e.g., Mathis et al. 1977; Draine and Lee 1984), as well as on a combination of stardust and interstellar medium dust (Greenberg and Hong 1974; Greenberg and Chlewicki 1984). Not too much credence should be given to such models since the interstellar extinction curve is quite insensitive to the exact composition of the dust. This is because other parameters (e.g., size distribution, shape, and surface roughness) will influence the extinction curve of an ensemble of particles as well. This non-uniqueness is compounded by the variations in the optical properties, even within one class of materials such as silicates, and the resulting uncertainty in the optical properties of interstellar grain materials. At this moment, the most diagnostic information on the dust composition results, therefore, from features in the extinction curve, notably in the infrared, rather than from the overall shape of the extinction curve.

In $\$ 1$, a compilation of the different dust components observed to be present in the interstellar medium and their relative importance is presented. These include the stardust components amorphous silicates, amorphous carbon, polycyclic aromatic hydrocarbons (PAHs), and graphite, 
as well as the interstellar medium components, which are the organic refractory grains and icy grain mantles. These data are summarized in Table I. It is emphasized that the uncertainties in these observations preclude a definite choice between the different proposed dust models, although it seems that stardust may dominate the dust composition in the solar neighborhood.

In $\$ 2$, the difference between amorphous and crystalline materials is examined. Some attention is given to the structure of truly amorphous materials (e.g., random network materials) such as glasses (e.g., silicates) and ices. The microcrystalline structure of soot particles is also discussed with an emphasis on the structural differences and similarities between graphite sheets, amorphous carbon particles, and PAH molecules. This section is concluded with a discussion of the structure of amorphous polymer solids, appropriate for the organic refractory dust component.

In $\$ 3$, the optical properties of amorphous and crystalline materials are contrasted. The emphasis is on silicates (amorphous), because the properties of carbon grains are to some extent already covered in the chapter by Allamandola, Tielens, and Barker in this volume. The optical properties of materials divide naturally into three regions which are discussed separately. The first is the optical and UV region, which is dominated by the electronic properties of the material. Section 3.1 focuses on understanding the observed, enhanced absorption at about $2 \mu \mathrm{m}$ of silicates formed in red giant envelopes. This absorption, which lies within the band gap of silicates, is due to localized electronic states, probably caused by the presence of impurities such as EeO in the silicate. The second is the mid-infrared region, which for amorphous materials is dominated by the fundamental vibrational modes of the nearest-neighbor atoms. Special emphasis is given in $\$ 3.2$ to the large width of the $10 \mathrm{\mu m} \mathrm{Si-O}$ stretching vibration in amorphous silicates. It is suggested that the decreased width of the $10 \mu \mathrm{m}$ feature in absorption as compared to. emission is due to the low temperature of the dust in the interstellar medium. The third is the far-infrared region, which shows the most distinctive differences between amorphous and crystalline materials. In $\$ 3.3$ the conditions for a $\lambda^{-1}$ far-infrared absorption efficiency are examined. From interstellar far-infrared observations it is concluded that the volume of the interstellar dust is dominated by layered materials, such as amorphous carbon or layerlattice silicates.

The physical principles of grain surface chemistry are discussed in \$4. This includes the surface structure of materials (\$4.1), the adsorption energy and residence time of species on a grain surface $(\$ 4.2)$, the sticking probability (\$4.3), the mobility of species on a grain surface $(\$ 4.4)$, the reactions that are of importance for the 
formation of icy grain mantles $(\$ 4.5)$, and the dissipation of the reaction heat $(\$ 4.6)$.

It is generally agreed that $\mathrm{H}_{2}$ is formed on grain surfaces in the interstellar medium. Because of its low mass, a physically adsorbed $H$ atom can tunnel through the activation barrier for reaction with a chemisorbed $\mathrm{H}$ atom and form $\mathrm{H}_{2}$. This process is examined in some detail in $\$ 5$.

In $\$ 6$ the depletion of elements due to their accretion on grain surfaces in the diffuse interstellar medium is examined. It is suggested that the high depletion of elements such as $\mathrm{Fe}$ and $\mathrm{Ca}$ is due to chemisorption on grain surfaces. Other atoms (e.g., $O$ and $N$ ) will also be chemisorbed, initially, but reaction with $H$ will form saturated hydrides which are easily photodesorbed. Their depletion will then be fairly low.

The composition of interstellar grain mantles is discussed in $\$ 7$. It results from a complex interplay of gas-phase and grain-surface reactions. Broadly speaking, three different regimes can be discerned, depending on the conditions in the gas phase. First, a reducing atmosphere in which most of the accreting gas-phase species are saturated through reactions with atomic hydrogen on the surface. Second, an oxidizing atmosphere in which surface reactions with atomic oxygen dominate. Third, an inert atmosphere in which the accreting gas-phase species essentially do not react with each other. Observations show that the reducing conditions dominate during the formation of interstellar icy grain mantles.

In $\$ 8$ the formation of the refractory dust component that is observed in the diffuse interstellar medium is discussed. Essentially, the problem is to get from the simple, largely hydrogenated molecules in icy grain mantles to more complex organic refractory material, which is able to survive better in the harsh environment of the diffuse interstellar medium. Some form of energetic processing of the grain mantle is required for this transformation. The most likely sequence of events in UV photolysis producing radicals, followed by the diffusion and reaction of these radicals to produce complex molecules. In order to get a substantial yield of organic refractory dust material, it is concluded that there has to be a significant internal UV field in molecular clouds and that transient heating mechanisms such as cosmic ray heating, which promote diffusion, are important.

Finally, in $\$ 9$ the contribution of grains to the gas-phase composition of molecular clouds is examined. It is emphasized that the critical temperature for grain-mantle formation is only about $30 \mathrm{~K}$ and that above this temperature grain-mantle formation is a slow process. Ejection mechanisms from grain surfaces are discussed in \$9.1. Transient heating of photolyzed grain mantles by cosmic rays or grain-grain collisions up to about $30 \mathrm{~K}$ promotes diffusion and reaction of stored radicals. The liberated reaction heat will explosively evaporate an 
appreciable fraction of the icy grain mantle. During this process more organic refractory dust material can be produced. The signatures of grain chemistry are discussed in $\$ 9.2$. It is pointed out that grain chemistry will mainly lead to simple hydrogenated molecules (e.g., $\mathrm{H}_{2} \mathrm{O}, \mathrm{NH}_{3}$, and $\mathrm{CH}_{3} \mathrm{OH}$ ), large deuteration effects (e.g., $\left.\mathrm{NH}_{3}: \mathrm{NH}_{2} \mathrm{D}: \mathrm{NHD}_{2}: \mathrm{ND}_{3} \approx 1: 0.1: 0.01: 0.001\right)$, saturated metal hydroxides (e.g., $\mathrm{NaOH}$, and ortho/para ratios characteristic for the dust temperature.

\section{COMPOSITION OF DUST IN DIFFUSE INTERSTELLAR MEDIUM}

Many different dust components have been proposed to be present in the diffuse interstellar medium. These include silicates, amorphous carbon, PAHs, graphite, organic refractories, SiC, metallic oxides and many more (cf. Mathis 1986 and references therein). Most models rely on some combination of these to explain the interstellar extinction curve. Direct information on the dust components present in the diffuse interstellar medium comes, actually, from structure in the extinction curve; for example, the 2200 \&ump, the 10 and $20 \mu \mathrm{m}$ features; several infrared absorption features in the spectrum of the galactic center; a $7.6 \mu$ absorption feature in some protostars; and the $3.3,6.2,7.7$, and $11.3 \mathrm{\mu m}$ emission features. A successful model should, of course, also explain the wavelength dependence of the observed extinction curve and, in particular, the amount of visual extinction per hydrogen atom $A_{v} / N_{H}$. These constraints can be used to identify the dust components present in the diffuse interstellar medium and to determine their relative importance. In the remainder of this section we present such an analysis of these constraints. It should be emphasized, however, that not all researchers in the field of interstellar dust will agree with our interpretation of the data, in particular with the details. The reader is referred to the review by Mathis (1986) for a critical analysis of some of the different dust models proposed.

The spectroscopic constraints listed above can be used to identify the different dust components present and to determine their relative importance. In line with most other analyses, we attribute the ubiquitous $2200 \AA$ bump and the 10 and $20 \mu \mathrm{m}$ features to graphite and silicate, respectively. Evidence for the presence of amorphous carbon, organic refractory material, and PAHs is provided by a $7.6 \mu \mathrm{m}$ absorption feature, several other absorption features, and the IR emission features, as detailed below. In Table I we summarize the estimates of the mass and volume per $H$ atom, $M_{j}$ and $V_{j}$, of each dust component identified in the interstellar medium. These have been determined from the observations using

$$
M_{j}=\sum_{i} f_{i} \times A_{i} \times m_{i}
$$




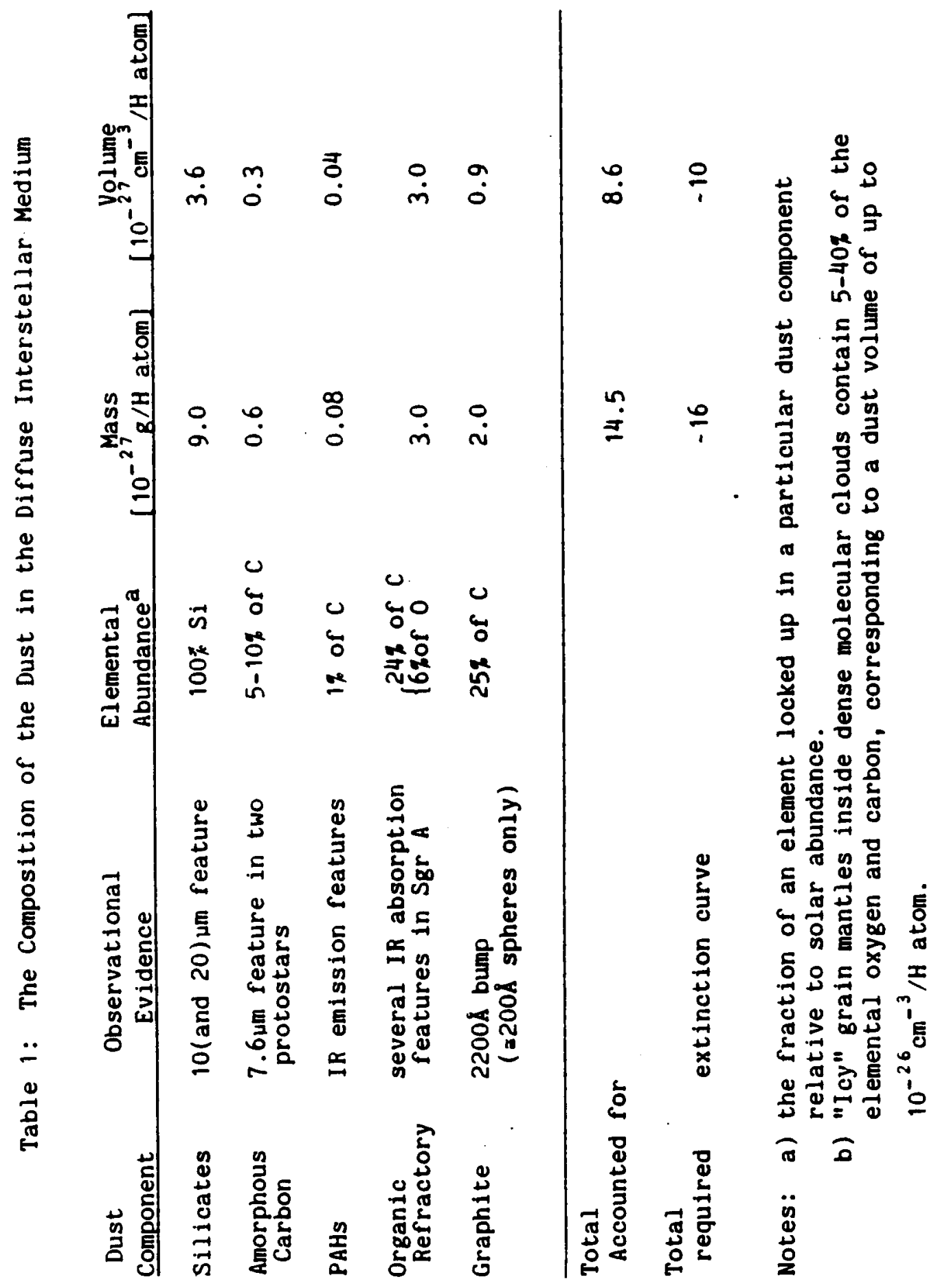


and

$$
v_{j}=M_{j} / p_{s},
$$

where $f_{i}$ is the fraction of element $i$ locked up in dust component $f ; A_{i}$ is the solar abundance of element $i ; m_{1}$ is the atomic mass of element $i$; $o_{s}$ is the specific weight of the dust component; and where the summation is over all elements in a dust component. The fraction of an element locked up in a particular dust component (relative to solar abundances) is estimated from the observed strength of the absorption (or emission) features per hydrogen atom. The details of this analysis are given below.

\subsection{Silicates}

The 10 and $20 \mu \mathrm{m}$ features are seen in many objects in absorption as well as emission, including oxygen-rich $M$ giants (presumably the birth sites), HII regions, protostars, and background stars (Merrill 1977; Aitken 1981). Generally, these features are attributed to the Si-0 stretching and bending vibrations in silicate materials. The width of the observed features (e.g., much broader than in terrestrial minerals) and the absence of substructure suggests that the silicates have an amorphous structure or are hydrated (Zaikowski et al. 1975; Day and Donn 1978; Day 1979).

The fraction of the silicon locked up in silicate dust grains in the diffuse interstellar medium can be estimated from the observed strength of the $10 \mathrm{\mu m}$ silicate absorption feature per magnitude of visual extinction. For eight bright, nearby ( $<3 \mathrm{kpc}$ ) WC Wolf-Rayets and supergiants, the ratio $\tau(10 \mu \mathrm{m}) / A_{v}$ is determined to be $18.5+1.5 \mathrm{mag}^{-1}$ (Roche and Aitken 1984). Assuming a standard dust-togas ratio, $\mathrm{N}_{\mathrm{H}} / \mathrm{A}_{\mathrm{Y}}$, appropriate for the diffuse interstellar medium of $1.9 \times 10^{21} \mathrm{mag}^{-1} \mathrm{~cm}^{-2}$ (Bohlin, Savage, and Drake 1978) and using the observed width of the $10 \mu \mathrm{m}$ feature of $220 \mathrm{~cm}^{-1}$, this translates into an integrated strength, $W(10 \mu \mathrm{m})$, of $6.2 \times 10^{-21} \mathrm{~cm}$ per hydrogen atom. Because of the disordered nature of amorphous or hydrated silicates, it is expected that all modes will be infrared-active and, thus, that the integrated strength of the Si-O stretching vibration will not vary much from one silicate material to the other (see \$3.2); this is borne out by laboratory studies. Laboratory measurements of the integrated strength of the $10 \mu \mathrm{m}$ feature in amorphous or hydrated magnesium silicates typically yield $1.2 \times 10^{-16} \mathrm{~cm}$ per Si atom (Penman 1976; Day 1979; Mooney and Knacke 1986). Note that the measured peak strength and width vary by a factor of 3 , but the integrated strengths agree to within $30 \%$. Using this value requires about $150 \%$ of the solar Si abundance to be locked up in silicates. Such an elemental enrichment in the present 
interstellar medium is perhaps not impossible. However, larger $10 \mathrm{\mu m}$ integrated absorption strengths have been reported for crystalline silicates $\left(2.8 \times 10^{-16} \mathrm{~cm}\right.$ per $\mathrm{Si}$ atom; Zaikowski, Knacke, and Porco 1975). Using this value requires that at least $63 \%$ of the silicon be locked up in silicate dust grains in the interstellar medium. Quite likely, the actual fraction of $S i$ locked up in silicates is about $100 \%$, and that is what we have assumed in Table $I$. Based on cosmic abundances and condensation sequences (in thermodynamic equilibrium; thus, perhaps not completely applicable) a magnesium or iron-magnesium silicate is expected to condense out in the outflow from late-type giants (Hackwell 1971). Some $\mathrm{Na}, \mathrm{Ca}$, and $\mathrm{Al}$ might also be contained in interstellar silicates. A composition of $(\mathrm{Mg}, \mathrm{Fe}) \mathrm{SiO}_{4}$ has been assumed in the calculation of the silicate mass per hydrogen atom. The specific density of terrestrial silicate materials ranges from about 2.5 to $3.5 \mathrm{~g} / \mathrm{cm}^{3}$. Interstellar silicate materials are probably amorphous and their specific density is on the low side of this range. In the calculation of the silicate volume per hydrogen atom (Table I) a specific density of $2.5 \mathrm{~g} / \mathrm{cm}^{3}$ has been assumed. Note that both these assumptions tend to maximize the contribution of silicates to the interstellar dust.

\subsection{Amorphous Carbon and Polycyclic Aromatic Hydrocarbons}

A broad and shallow 7.6 um absorption feature has been observed in the spectrum of NGC 7538-IRS9 and W33A, two protostars with the largest known $10 \mu \mathrm{m}$ optical depth (Tielens et al. 1986a). This band may be due to the CC stretching vibration in amorphous carbon dust particles, although other interpretations (in terms of molecules in icy grain mantles) are also possible. The hydrogen column density or visual extinction toward these sources is not known, but from a comparison with the $10 \mu \mathrm{m}$ optical depth and assuming that all of the $\mathrm{Si}$ is in the form of silicate grains, the fraction of the carbon locked up in amorphous carbon grains is calculated to be 5-10\% (Tielens et al. 1986a). If the silicon depletion in silicate grains is actually less, then this number has to be revised downward accordingly. The resulting mass and volume estimates (assuming a specific weight of $2 \mathrm{~g} / \mathrm{cm}^{3}$ ) for amorphous carbon are given in Table I.

Emission features at $3.3,6.2,7.7$, and $11.3 \mu \mathrm{m}$ have been observed in a variety of sources, including planetary nebulae, HII regions, reflection nebulae, and galactic nuclei (Russell, Soifer, and Willner 1977; Aitken and Roche 1984; Sellgren, Werner, and Dinerstein 1983; Cohen et al. 1986). These features have been attributed to vibration involving polycyclic aromatic hydrocarbons in various forms (Duley and Williams 1981; Leger and Puget 1984; Allamandola, Tielens, and Barker 1985). From an analysis of the emission mechanism, the fraction of carbon locked up in these molecules is estimated to be about $1 \%$. For 
completeness, this component is included in Table I. It is, however, likely that this component is just the molecular domain of the size distribution of interstellar amorphous carbon grains (see $\$ 3.5$ ).

\subsection{Organic Refractory Material}

Absorption features due to an organic refractory dust component have been detected along the line of sight toward the galactic center (Willner et al. 1979; Allen and Wickramasinghe 1981; Butchart et al. 1985; Tielens et al. 1986b). The dust along the line of sight toward the galactic center is presumably located in the diffuse interstellar medium (Roche and Aitken 1985). From an analysis of these spectra in terms of functional groups of simple molecules, the fraction of the carbon and oxygen locked up in this dust component has been determined to be $24 \%$ and $6 \%$, respectively (Tielens et al. 1986b). The $H$ column density has been determined from the estimated visual extrinction, $A_{y}=30 \mathrm{mag}$, assuming a standard dust-to-gas ratio. The specific density of this dust component has been assumed to be $1 \mathrm{~g} / \mathrm{cm}^{3}$.

It should be emphasized that infrared spectra generally only yield the characteristic molecular subgroups responsible for the absorption and not the specific molecule. The intrinsic absorption strength of these molecular subgroups, and thus the calculated column density, is therefore somewhat uncertain (by a factor of 2). Furthermore, it should be noted that the silicate abundance in the galactic center is larger than in the local interstellar medium (Roche and Aitken 1985); this may also pertain to the organic refractory component. In fact, the $3 \mathrm{um}$ spectrum of the supergiant VI Cygni No. $12(-3 \mathrm{kpc})$ does not show the $3.4 \mu \mathrm{m}$ absorption feature $\left(\tau(3.4 \mathrm{\mu m})<0.03 ; A_{v}=10\right.$ mag; Gillett et al. 1975a). Assuming that the composition of the organic refractory dust in the local interstellar medium is similar to that along the line of sight to the galactic center, we arrive at an upper limit on the fraction of the carbon locked up in the dust component of about $10 \%$. Clearly, the volume and mass values given in Table I should only be considered as order-of-magnitude estimates.

\subsection{Graphite}

The strong 2200 \&ump, which is a ubiquitous component of the interstellar extinction curve in the diffuse interstellar medium (cf. the review of Savage and Mathis 1979 and references therein), is generally attributed to small ( $-200 \AA$ ) spherical graphite grains (Gilra 1972). About $25 \%$ of the available carbon is required to explain the strength of this feature (Tielens and de Jong 1979). Note that larger carbon grains show no detectable absorption features, and their abundance can presently only be guessed at. A specific density of $2.2 \mathrm{~g} / \mathrm{cm}^{3}$ has been 
assumed for graphite. There are some problems in attributing the $2200 \AA$ bump to graphite particles. The graphite transition is intrinsically very strong and, because of the surface polarization charge, the shape and peak position are very sensitive to shape and size (an excellent review of the effects of the surface polarization charge on the spectrum of small particles is given by Bohren and Huffman 1984). The observations imply 200 spherical graphite grains. Moreover, there may be some problems with graphite in explaining the observed variations in the ratio of the FUV extinction $(\lambda<1600)$ to the $2200 \AA$ bump (Greenberg and Chlewicki 1984). For these reasons, the $2200 \AA$ bump has sometimes been attributed to amorphous carbon instead of graphite grains (cf. Mathis 1986). (The structural difference between amorphous carbon and graphite will be discussed in $\$ 3.5$ ). Indeed, the intrinsic strength of the amorphous carbon transition is much less (Borghesi et al. 1983), and surface charge effects are, therefore, unimportant. The drawback of amorphous carbon is, however, that more material is required to explain the observed strength of the $2200 \AA$ feature per hydrogen atom. This does not only conflict with the amount of amorphous carbon determined from the infrared, but actually requires more than the cosmic abundance of carbon.

\subsection{Total Extinction}

A rough estimate of the total volume of dust material required to explain the observed extinction can be made from a Kramers-Kronig analysis of the extinction curve (Purcell 1969). An extensive discussion of this analysis is given in Spitzer (1978). For reasonable values of the optical properties of the dust, the value in Table I results. This is only somewhat larger than the sum of the identified components. A more detailed analysis of the extinction curve is, however, required to determine whether the identified components can indeed explain the full extinction curve.

\subsection{Interstellar Grain Mantles}

For completeness we now mention interstellar grain mantles. Inside dense molecular clouds, interstellar grains can acquire an icy grain mantle, consisting of such simple molecules as $\mathrm{H}_{2} \mathrm{O}, \mathrm{CH}_{3} \mathrm{OH}, \mathrm{CO}$, and $\mathrm{NH}_{3}$ (Merrill et al. 1976; Hagen et al. 1980; Knacke et aI. 1982; Lacy et al. 1984; Tielens et al. 1984, 1986a). The fraction of the carbon and oxygen locked up in these grain mantles ranges typically from $1-40 \%$ with a corresponding mass of $0.2-10 \times 10^{-27} \mathrm{~g}$ per $\mathrm{H}$ atom and corresponding volume of $0.2-10 \times 10^{-27} \mathrm{~cm}^{3}$ per $\mathrm{H}$ atom. Thus, the growth of these icy grain mantles in molecular clouds can actually double the total dust mass or volume. However, these icy grain mantles have never been 
observed outside of molecular clouds. Presumably, these volatile materials are easily destroyed by interstellar shocks and UV photodesorption and their abundance in the diffuse interstellar medium is, therefore, low (Barlow 1978; Draine and Salpeter 1979); as a result, they are not included in Table I.

\subsection{Discussion}

In the preceding paragraphs different dust components in the diffuse interstellar medium have been identified (cf. Table I). These are silicates, amorphous carbon, PAHs, organic refractory materials, and graphite. From Table I, one may get the impression that the organic refractory dust component and silicates dominate the composition of the interstellar dust rather than graphite. Greenberg and his co-workers (Greenberg and Hong 1974; Greenberg and Chlewicki 1984) have proposed such a model for the interstellar dust. It should be borne in mind, however, that the estimate of the total contribution of the organic refractory component is actually quite uncertain; it may be much less than estimated, particularly in the solar neighborhood. If that is the case, the bulk of the dust has to be in a form without detectable IR or UV absorption features. Large graphite particles fulfill these conditions. The electronic intraband transitions in graphite screen the (weak) lattice vibrations of the carbon atoms and make them presently undetectable (Draine 1984). Furthermore, if their size is large enough ( $>0.1 \mathrm{\mu m})$ large graphite particles will have an extinction cross section in the UV equal to the geometric cross section and, therefore, will not show a detectable UV feature. Thus, if the volume of the organic refractory component is much less than estimated in Table I, then large graphite particles without detectable absorption features (a theorist's dream!) may dominate the composition of the interstellar dust. Models based on this premise have been developed by Mathis and his co-workers (Mathis, Rumpl, and Nordsieck 1977; Mathis and Wallenhorist 1981) and by Draine and Lee (1984). Choosing between these two possible models is presently not possible. As emphasized before, the most uncertain component, besides graphite, is the organic refractory material. Determining its composition and, hence, its volume will require studies of the infrared absorption features along many different lines of sight, in particular in the local diffuse interstellar medium. Nevertheless, it should be emphasized that all the materials identified in Table I are important components of the interstellar dust and contribute substantially to the interstellar extinction curve in at least some part of the spectrum. In our opinion, it is quite likely that the composition of the interstellar dust lies somewhere in between the two extremes represented by the models of Greenberg and Mathis. 


\section{THE DISORDERED STRUCTURE OF INTERSTELLAR DUST}

Interstellar grains are generally thought to be highly disordered. Th:s assessment is based on two observations. First, the width of the $10 \mathrm{\mu m}$ silicate feature is much larger than that in crystalline silicate materials. This broadening can result from the disorder present in amorphous or hydrated silicates (Zaikowski et al. 1975; Day and Donn 1978; Day 1979). Second, the far-infrared cross section of the interstellar dust seems to fall off much slower with wavelength than expected for crystalline solids. Laboratory studies suggest that this is also a characteristic of some amorphous materials. Theoretically, disordered structures are expected because grain condensation generally occurs under nonequilibrium conditions. In this section we will examine the structure of interstellar grains with emphasis on amorphous silicates. A detailed discussion on the structure of amorphous materials and their properties can be found in the Ehrenreich report (1972) and in the monograph by Zallen (1983).

\subsection{Structure of Amorphous Solids}

Amorphous solids, sometimes referred to as glasses, are characterized by complete chemical coordination (i.e., few dangling bonds), as are crystalline solids. Similarly, the nearest-neighbor distances (i.e., bond lengths) are nearly constant. There is, however, a considerable spread in bond angles. Because of this latter point, amorphous solids do not have long-range order, in contrast to crystalline solids. This structural difference between amorphous and crystalline solids is illustrated schematically in Figure 1. In the crystal, the nearest-neighbor equilibrium distances and the bond angles are all equal. Consequently, it possesses long-range order and translational symmetry. The atomic arrangement in an amorphous solid is a continuous random network that lacks symmetry and periodicity (Zachariasen 1932). Specifically, the nearest-neighbor distances are only almost equal. Moreover, there is considerable spread in the bond angles. Clearly, there is a considerable amount of local order but a complete absence of long-range order in the amorphous state (cf. Fig. 1). The presence of the short-range order is, of course, fust a consequence of the chemical bonding of the solid.

Although not strictly applicable to interstellar solids, it may be advantageous from a conceptual point of view to study a schematic volume-temperature phase diagram (Fig. 2). When the temperature is lowered, a gas will condense to the liquid phase at the boiling temperature, $T_{b}$. Essentially, this is a transition from a regime where the intermolecular forces are unimportant to one in which they dominate. Although the free-volume (i.e., the volume available to each molecule) is much less in the liquid phase than in the gas phase, it is still 


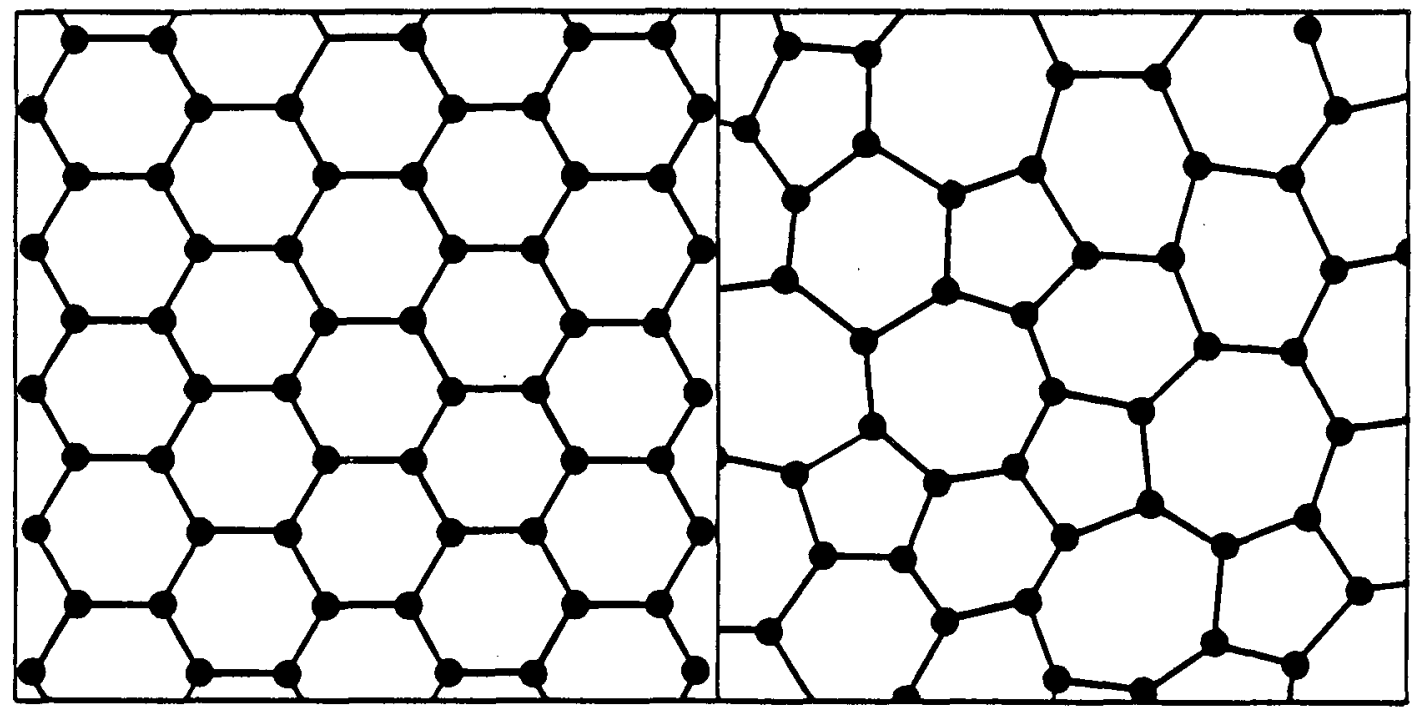

Figure 1. Atomic arrangement in a crystalline and an amorphous solid. The dots indicate the equilibrium positions about which the atoms vibrate, and the lines indicate the chemical bonds.

sufficiently large to allow translational motion to the molecules. A molecule in the liquid phase is continuously making and breaking bonds with its neighbors while it is diffusing through the liquid. This is, of course, what gives a liquid its macroscopic fluid character. Thus, because of the chemical bonding of the molecules, a liquid will have considerable short-range order. But, because of the continuous rearrangement of the molecules, the bond angles and bond lengths will show a broad distribution around the average value. Consequently, there is no long-range order in a liquid.

When a liquid is very slowly cooled to the freezing temperature, it will transform to the crystal phase (route 1 in Fig. 2). That is, crystallization centers will form and will grow at the crystal-liquid interfaces. Like the gas-liquid transition, this process is accompanied by an abrupt change in the free-volume available to the molecules. In the crystal, the molecules are arranged on a translationally periodic lattice, which exhibits long-range order. It is important to realize that the crystallization process is essentially a kinetic one. That is, during the nucleation and growth the molecules have to "find" each other and arrange themselves on the crystal lattice in the energetically most favorable orientation. This takes time. Thus, if the liquid is rapidly cooled below its freezing temperature nucleation cannot take place and the liquid phase will persist (i.e., it will form a supercooled liquid). 


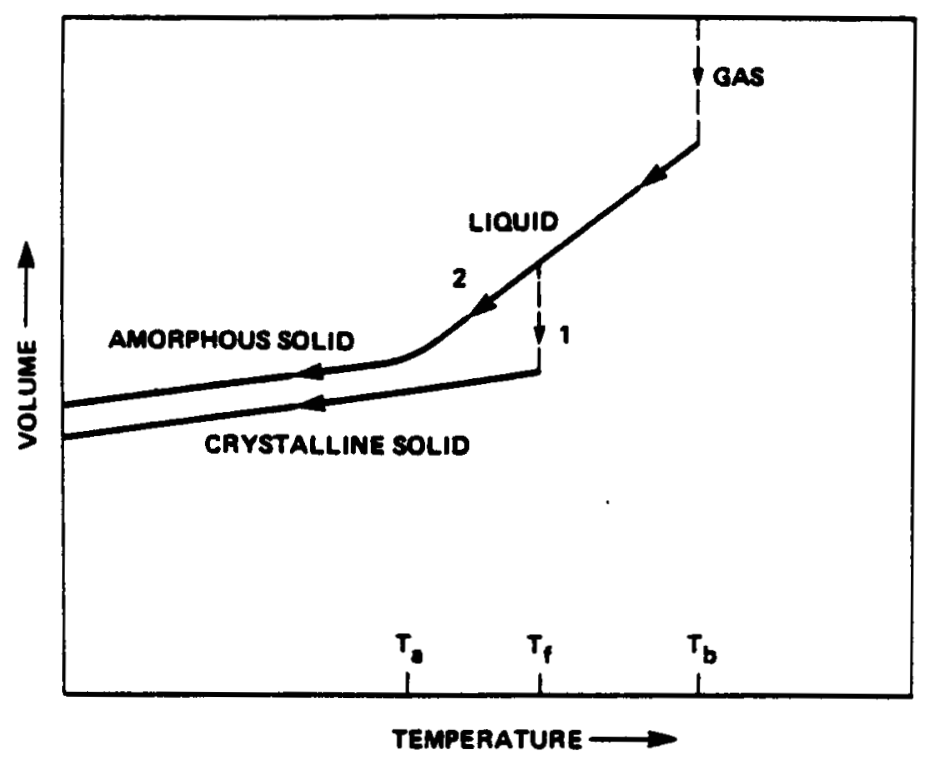

Figure 2. Volume-temperature phase diagram. There are two ways for the condensation of a solid to proceed: route 1 is the path to the crystalline state; route 2 is the rapid-quench path to the amorphous state.

When a fluid is cooled below its freezing temperature while nucleation is prevented (i.e., rapid cooling; route 2 in Fig. 2), the freevolume available to its molecules will shrink further and further because of the thermal contraction (i.e., the anharmonicity of the intermolecular potential). It will finally reach a point where the available free-volume does not allow any further translationary motions of the molecules and the fluid has been transformed to an amorphous solid. The molecules are "stuck" together in a rather random way. The molecules will now only show oscillatory motion around a well-defined equilibrium position. The temperature at which this transformation occurs, $T_{a}$, depends slightly on the cooling history of the solid, because the rate of kinetic processes depends on the temperature. In contrast to the gas-liquid and liquid-crystal transformations, this transition is characterized by a smooth volume change. Because of the decreased free-volume of the molecules the distribution of the bond lengths in the amorphous state will be much less broad than in the liquid state, resembling more the crystalline state. The distribution of bond angles however is very broad, similar to that in the liquid. state. Consequently, although there is short-range order, there is no long-range order.

It is thus important to realize that any liquid or gas when cooled rapidly enough (i.e., nucleation prevented) will form an amorphous solid. This amorphous state is generally metastable with respect to the 
crystalline state, but the latter is kinetically inaccessible. The detailed structure of a particular amorphous material will depend on the details of its formation. In particular, if the cooling is very rapid, the amorphous solid may retain the local structure of the liquid $(e .8 .$, the presence of highly strained bond angles). In contrast, when the cooling is somewhat slower, the amorphous solid may be able to rearrange itself somewhat and attain a thermodynamically slightly more favorable structure in the amorphous state. It should be emphasized that there is not just one amorphous state but many, each with a slightly different energy. In fact, there is an almost continuous distribution of amorphous states with energies up to $\mathrm{KT}_{a}$, where $T_{a}$ is the temperature for the liquid-amorphous solid transition. Thus, although amorphous solids may approach an "ideal" metastable noncrystalline state, they can be quenched into many other metastable states. The structure and properties of an amorphous solid depend, therefore, also on the preparation conditions.

Such a distribution of amorphous states will be even more prominent for the condensation conditions prevailing in interstellar space. In that case, condensation will take place directly from the vapor state to the solid state. Often the temperature of the system will be much less than the transition temperature, $T_{a}$, from the amorphous to the crystalline state (note that in this case, this transition does not go through the liquid phase and the temperature $T_{a}$ differs from that defined above). Accreting molecules will, thus, have insufficient energy to arrange themselves favorably and the resulting structure will be far from that reached by cooling down from the energetically more favorable structure of an amorphous solid deposited at a temperature around $T_{a}$. Upon warm-up, an amorphous structure rapidly deposited at a low temperature can rearrange itself somewhat to an energetically more favorable amorphous structure. This annealing process will continue until the temperature at which it transforms into the crystalline phase is reached. This annealing process for the interstellar dust has been studied experimentally in considerable detail for amorphous $\mathrm{H}_{2} \mathrm{O}$ ice (Hagen, Tielens, and Greenberg 1981) and in somewhat less detail for amorphous silicates (Day and Donn 1978; Nuth and Donn 1982).

\subsection{Impurities and Defects}

Up to now we have only discussed disorder caused by condensation below the amorphous-crystalline transition temperature. Disorder can, however, also be due to the presence of impurities and defects in the crystal. Impurity atoms or molecules will have slightly different sizes, polarizabilities, bond strengths, and angles than the host species. Their presence will necessitate some degree of local rearrangement of the crystal to accommodate them. This is, of course, 
particularly true for an impurity ion with a valence different from the host in an ionic crystal. Impurities will, thus, distort the surrounding host species (slightly) and thereby destroy the long-range order of the crystal. For icy grain mantles formes in interstellar molecular clouds this type of disorder is very important because these mantles consist of about equal parts of $\mathrm{H}_{2} \mathrm{O}$ and $\mathrm{CH}_{3} \mathrm{OH}$ (or another alcohol) and traces of other molecules such as $\mathrm{CO}$ and $\mathrm{NH}_{3}$ (Tielens et al. 1986a). Of course, such icy grain mantles are formed at temperatures $(\sim 10 \mathrm{~K})$ much below $T_{a}$ and would, therefore, be highly disordered even if they had a homogeneous composition.

Crystal lattices will also exhibit defects, owing to the presence of vacancies in lattice positions (Schottky defects) or the promotion of molecules or ions from substitutional to interstitial sites (Frenkel defects). The formation of these defects in a crystalling solid has an appreciable activation barrier. In interstellar space they might result from cosmic ray bombardment. A concentration of point-defects may give rise to dislocations (screw or edge dislocations). Dislocations are generally a product of the nucleation and growth processes of crystalline solids. In fact, a crystalline solid generally grows at these dislocations. For an amorphous solid, formed under supercooled conditions, dislocations are, however, of little importance.

\subsection{Amorphous Silicates}

Silicate glasses (e.g., fused quartz) are the prototype of (binary) amorphous solids, and their structure is well represented by a continuous random network in three dimensions (the three-dimensional analog of Fig. 1; Zachariasen 1932). In fused quartz, each silicon atom is predominantly covalently bonded to four nearest-neighbor oxygen atoms, and each oxygen atom is bonded to two nearest-neighbor silicon atoms. Each of the $\mathrm{SiO}_{4}$ units consists of an almost regular tetrahedron, and most of the structural variations are due to variations in the $\mathrm{Si-O-Si}$ bond angles (Bell and Dean 1972).

The presence of electropositive elements such as $\mathrm{Mg}$ changes this picture somewhat. There are now two kinds of oxygen atoms: bridging oxygens, which link two tetrahedrons together, and non-bridging oxygens, which belong only to one tetrahedron. These non-bridging oxygens are bonded to the electropositive element by charge ransfer and are topologically "dead-ended." For a small number of cations (positive ions), the resulting structure is that of a polymer with long chains cross-linked at intervals and cations in holes in between the oxygen tetrahedrons. This is illustrated in Figure 3a. When the number of bridging oxygen atoms becomes less than two per tetrahedron, spatial coherence by crosslinking is not possible. The silicate structure consists now of isolated chains ( $c f$. Fig. 3b). If the number of cations present increases 
even further, full charge separation may take place and each tetrahedron is only bonded to cations. In any case, however, the disorder in silicate glasses is due to the variable orientation of the $\mathrm{SiO}_{4}$ tetrahedrons with respect to each other. Additional disorder can be caused by the presence of different cations in the structure.

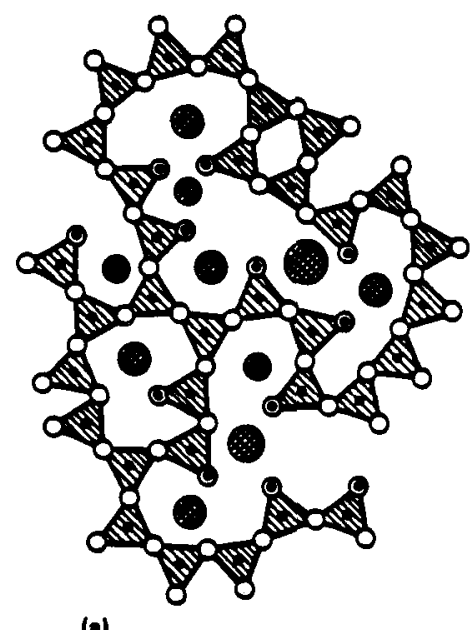

(a)

$$
\begin{aligned}
& \text { - SILICON } \\
& \text { O BRIDGING OXYGEN } \\
& \text { O NON-BRIDGING OXYGEN } \\
& \text { CATION }
\end{aligned}
$$

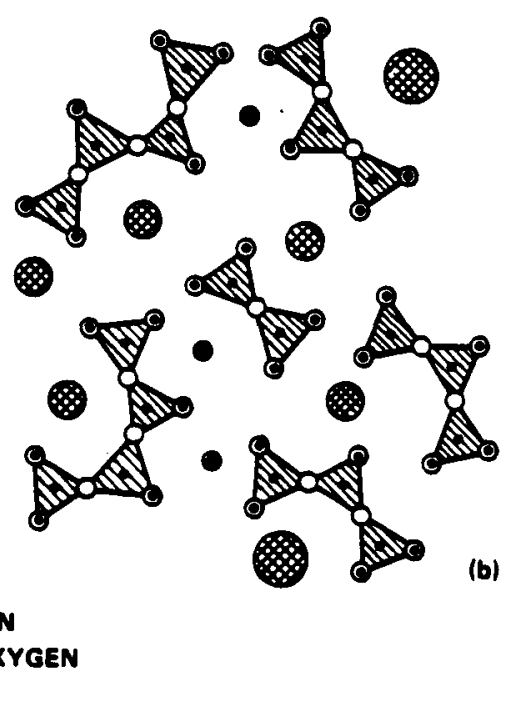

Figure 3. Two-dimensional representation of the atomic arrangement in silicate glasses. Each silicon atom is in the center of an oxygen tetrahedron. Some oxygen atoms bridge two tetrahedrons whereas others are bonded to cations. Depending on the number of cations present the $\mathrm{SiO}_{4}$ chains will be cross-linked (a) or not (b).

\subsection{Amorphous $\mathrm{H}_{2} \mathrm{O}$ Ice}

The structure of amorphous $\mathrm{H}_{2} \mathrm{O}$ ice is very similar to that of silicates discussed above (Madden et al. 1978). Its infrared spectrum behaves very similar to that of silicates except for effects owing to hydrogen bonding. This has been discussed extensively in an astrophysical context by Hagen, Tielens, and Greenberg $(1983 \underline{a}, \underline{b})$. Here we will merely examine the importance of the thermal history for the structure of interstellar amorphous ice. Consider the condensation of $\mathrm{H}_{2} \mathrm{O}$ molecules on preexisting silicate cores in the outflow from oxygen-rich late-type giants. For the typical vapor pressures of $\mathrm{H}_{2} \mathrm{O}$ in these outflows, the condensation temperature of $\mathrm{H}_{2} \mathrm{O}$ is about $90 \mathrm{~K}$ (Nakagawa 1980; that is, at this temperature the accretion rate equals the evaporation rate). The time-scale for the amorphous-crystalline transformation is given by a Boltzman expression: 


$$
\tau=v^{-1} \exp \left(E_{a} / k T\right)
$$

where $E_{a}$ is the activation energy for the transformation and $v$ is a characteristic frequency. Because the disorder in amorphous $\mathrm{H}_{2} \mathrm{O}$ ice is mainly a result of a broader distribution of bond angles (Maddan et al. 1978), it is appropriate to use the $\mathrm{O}-\mathrm{H}$ bending frequency $\left(5 \times 10^{13} \mathrm{~Hz}\right)$ for v. The time-scale for transformation observed in the laboratory, $45 \mathrm{~min}$ at $140 \mathrm{~K}$ (Hagen, Tielens, and Greenberg 1981), translates then into an activation energy of about $5500 \mathrm{~K}$. This is, of course, somewhat less than the binding energy of an $\mathrm{H}_{2} \mathrm{O}$ molecule in an $\mathrm{H}_{2} \mathrm{O}$ ice $(-8000 \mathrm{~K})$ and actually very similar to the heat of formation of the crystalline state from the amorphous state. At a temperature of $90 \mathrm{~K}$, appropriate for late-type giants, the transformation time-scale is then about $2 \times 10^{5} \mathrm{yr}$. This is much longer than the time-scale for the growth of the ice mantle. It is also much longer than the cooling time-scale of the grains owing to the outflow $\left(-10^{3} \mathrm{yr}\right)$. The ice will condense in the amorphous form and will not be able to transform to the crystalline state. Thus, although the amorphous state is metastable with respect to the crystalline state, the latter is kinetically inaccessible. It is, thus, not surprising that the $3 \mu \mathrm{m} \mathrm{H}_{2} \mathrm{O}$ ice band detected in the spectrum of the late-type giant OHO739 has a shape characteristic for amorphous $\mathrm{H}_{2} \mathrm{O}$ ice at about $80 \mathrm{~K}$ (Hagen, Tielens, and Greenberg 1983b).

\subsection{Polycrystalline Carbon Grains}

A polycrystalline solid consists of a large number of microcrystals (10-100 $\AA)$ separated by some connective material. Within the microcrystals the molecules are positioned on a regular lattice as in a crystalline solid. The connective material at the grain boundaries is highly disordered ( $\mathrm{Fig}$. 4). The microcrystalline description of amorphous solids did have some popularity in the past, in particular for glasses, but has now generally been deserted for the noncrystalline description shown in Fig. 1. The two descriptions differ, of course, in the extent of the local order. It should be emphasized that fine-grained polycrystalline solids certainly exist in nature, but experiments have shown that in general amorphous solids are not microcrystalline (Zallen 1983; Phillips 1981). As will be discussed below, interstellar grains may have-a polycrystalline structure because of their formation history. Eigure 5 shows schematically the structure of a carbon (soot)

particle. This is essentially a polycrystalline structure. On a small scale (diameter < 50 ) the carbon atoms are arranged on a planar honeycomb lattice, as is graphite. The carbon atoms are bonded by regular covalent $\mathrm{sp}^{2}$ bonds to three neighbors. These bonds are localized. The fourth electron of each carbon atom is delocalized among the $\pi$ bonds 


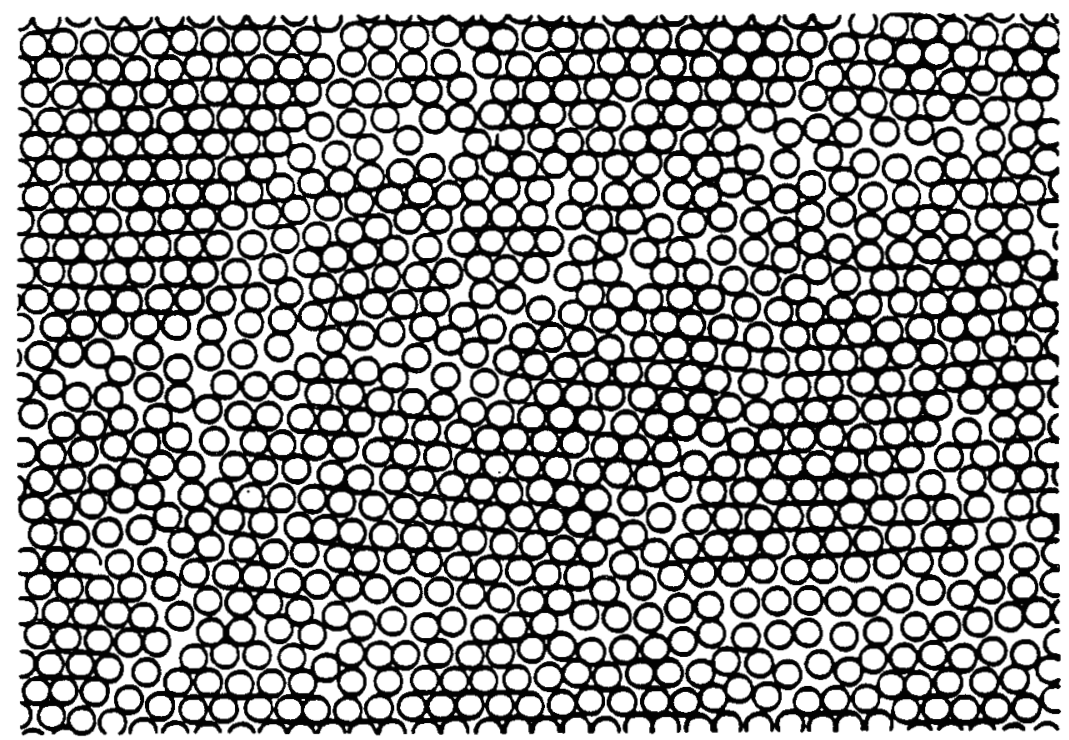

Eigure 4. Structure of a polycrystalline material. Highly ordered microcrystals are separated by highly disordered connective material. Note that unlike the amorphous structure shown in Figure 1, there are large regions of fairly ordered material present.

and resonates between several valence-bond structures, giving each $C-C$ bond a one-third double-bond character. These small planar "crystals" are in fact just the carbon skeleton of polycyclic aromatic hydrocarbons (PAHs). Typically, they are less than $50 \AA$ in diameter. Several of such PAHs are stacked together in platelets bonded by weak van der Waals forces, as are the sheets in graphite. Often there is also a considerable content of disorganized, tetrahedrally bonded, $\mathrm{sp}^{3}$ carbon (i.e., diamond structure), which distort the planar structure and can link different layers within a platelet. Functional groups at the edge of these platelets can also cross-link different layers within a platelet. It is these functional groups that separate different platelets and prevent orientation of the platelets with respect to each other in a soot particle. Although these platelets are topologically similar to graphite, they will have quite different optical properties (cf. Allamandola, Tielens, and Barker, elsewhere in this volume). These platelets are assembled into a much larger, disordered unit, the soot particle, bonded by weak van der Waals forces or by bridging aliphatic hydrocarbon groups (i.e., tetrahedrally bound carbon skeletons rather than aromatic skeletons). As in an amorphous solid, there is thus an absence of long-range order, although the structural units themselves have highly regular, planar hexagonal structures. 
<smiles>c1ccccc1</smiles>

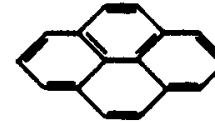

POLYCYCLIC AROMATIC HYDAOCAADON

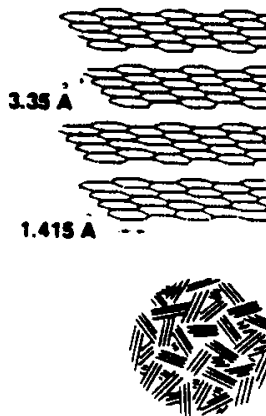

PLATELET

SOOT PARTICLE

Figure 5. Structure of a soot particle. The atoms are arranged on a planar honeycomb lattice. Each layer can be viewed as a small graphite sheet or the carbon skeleton of a large polycyclic aromatic hydrocarbon (PAH) molecule. The structure of benzene, the smallest PAH, is shown in detail. Several of these layers form a platelet. These platelets are the building blocks of soot particles.

It should be emphasized that carbon rapidly condensed on a substrate will form an amorphous solid with aromatic and tetrahedrally bound carbon atoms "randomly" interspersed. The differences in bond length and angle will produce a disordered structure similar to that shown in Figure 1. The soot particles described above are from a structural point of view intermediate between this "truly" amorphous carbon and graphite.

These two different views of interstellar amorphous carbon grains merely reflect a difference in the condensation history. The polycrystalline particles of Figure 5 will form when many condensation nuclei (the PAHs) are formed simultaneously in a (slowly) condensing gas. Most of the carbon in the gas will crystallize out into these nuclei. Farther downstream in the outflowing wind these PAHs can then coagulate, or cluster, into the much larger soot particles. In contrast, truly amorphous carbon can be formed when only a few condensation nuclei (i.e., PAHs) are formed in a rapidly condensing gas. The condensation of the remainder of the carbon forms large grains with an amorphous structure. This discussion is, of course, very general, since it is likely that the condensing species are molecules or ions rather than carbon 
atoms. The high abundance of PAHs with about 20 carbon atoms in planetary nebulae suggests that the first process dominates in that case (Allamandola, Tielens, and Barker 1986). The presence of only weak IR emission features in the population I WC 9 Wolf-Rayets stars, suggests that the second process may actually dominate in these outflows (Cohen et al. 1986). In any case, the differences between amorphous carbon and polycrystalline carbon are too subtle to be of importance at the present state of our knowledge of interstellar carbon grains. However, as the observations and theory progress, we anticipate that these differences will be revealed.

\subsection{Macromolecular Organic Refractory Grains}

The organic refractory component of the dust in the diffuse interstellar medium contains methyl $\left(\mathrm{CH}_{3}\right)$, methylene $\left(\mathrm{CH}_{2}\right)$, carbonyl $(\mathrm{C}=0)$ and probably alcohol $(\mathrm{OH})$ groups. The relative abundance of these groups suggests that the molecules have a highly branched carbon skeleton or are fairly small (Tielens et al. 1986므). The latter possibility is, however, ruled out by the requirement that they have to be able to survive in the harsh environment of the diffuse interstellar medium. The molecular structure suggested by the IR data is that of a copolymer ( $c$. Fig. 6). The side groups in this chain can be a hydrogen atom or a $\mathrm{CH}_{3}$, $C=0$, or $\mathrm{OH}$ group in random order. Also an occasional backbone $C$ atom could be in the carbonyl $(C=0)$ form. Typically, a polymer contains about $10^{4}$ backbone $C$ atoms but the polymer length is not well known in the interstellar case.

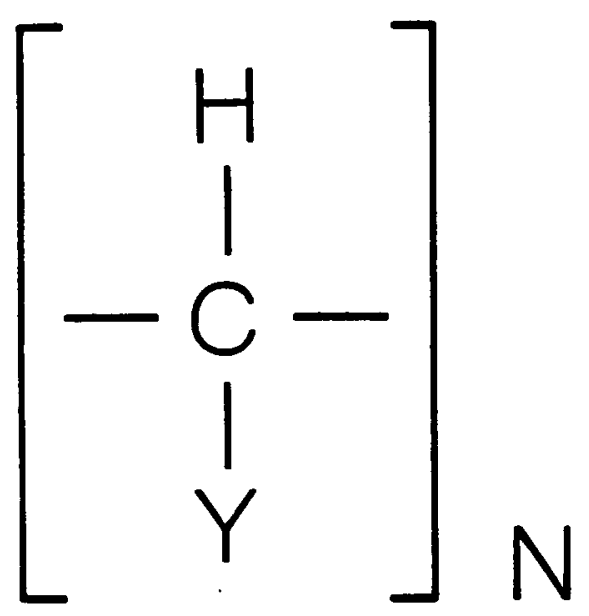

Figure 6. Monomer repeat unit within a polymer chain. The carbon atoms in these units form a linear chain; $Y$ indicates a side group. 
The structure of such a solid polymer will be very disordered. Unlike crystalline solids or monoatomic solids there is no repeatable sub-unit (the unit cell). Figure 7 represents a perspective sketch of a small section of a polystyrene polymer to give an impression of the spatial disorder. In an isolated polymer all the bond lengths and bond angles are equal to the crystalline value. Even the third nearest neighbors have the correct crystal separation (i.e., staggered bond configuration). This set of crystal-like requirements is far stricter than for the amorphous structures discussed before (cf. Fig. 1). Yet, a polymer has a great deal of configurational freedom, which has been used here to open up the polymer as much as possible to show its molecular structure clearly. Obviously, a polymer is quite flexible and can change directions very often along its chain by rotation about a backbone bond. In that sense, Figure 7 is somewhat misleading since it may give the incorrect impression that an amorphous polymer solid consists of aligned strands of polymer molecules. In fact, such a solid has a random-coil structure, as illustrated in Figure 8. Each polymer adopts a random-walk configuration and the structure of the organic glass consists, thus, of many intertwined random-coils. Note that the scale of Figure 8 is about 1000 times larger than that of Figure 7 . The bonding between different polymer strands within an amorphous polymer solid is due to weak van der Waals forces or hydrogen bonding forces between side groups. It should be emphasized that we expect that because of their chemical formation process, the polymers in the organic refractory component of the interstellar dust are not linear, onedimensional molecules, but rather very branched, three-dimensional molecules. That is, they are cross-linked by true chemical bonds between side groups (e.g., resins). For that reason they are more properly called macromolecules.

\section{OPTICAL PROPERTIES. OF AMORPHOUS MATERIALS}

The amorphous nature of materials has considerable influence on their physical properties, such as specific heat and thermal conductivity, at very low temperatures (Phillips 1981). Essentially, this is due to the existence of more than one amorphous state very close in energy. Tunneling between these states can then be of importance at very low temperatures. Generally, this is not very important at interstellar temperatures $(>10 \mathrm{~K})$. However, the disordered structure of a solid is of considerable importance for its optical properties, even at higher temperatures. As mentioned in the introduction to $\$ 2$, it is generally thought that a large fraction of the interstellar dust has an amorphous structure. This is based on the shape of the $10 \mu \mathrm{m}$ feature and on the far-infrared absorption law $\left(\sim \lambda^{-1}\right)$. The high near-infrared absorption 


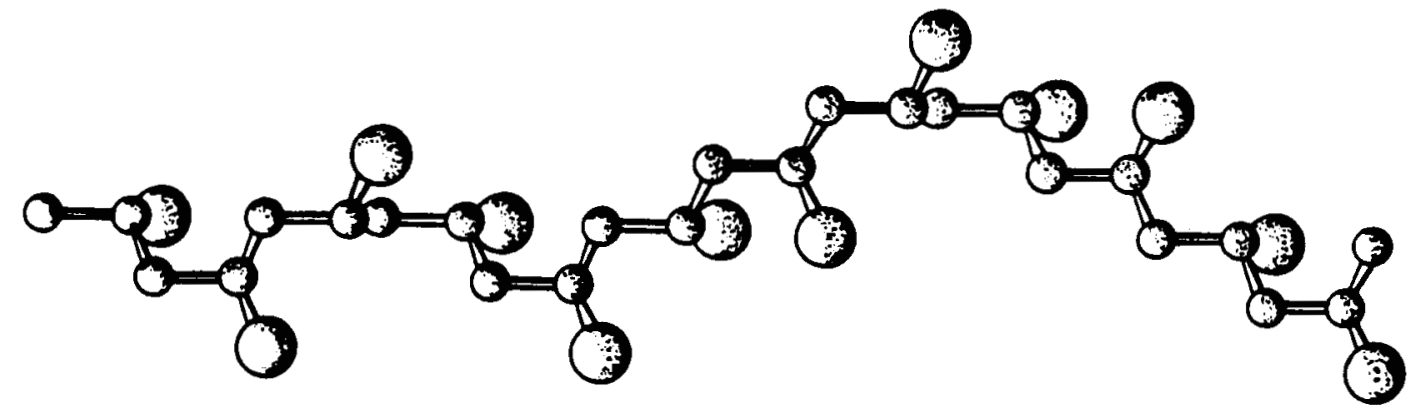

CARBON ATOM

SIDE GROUPS

Figure 7. Small section of a polystyrene polymer. The carbon atoms are tetrahedrally bonded. Alternating carbon atoms in this polymer contain a side group (a benzene ring in this case). For clarity, the hydrogen atoms have been left out.

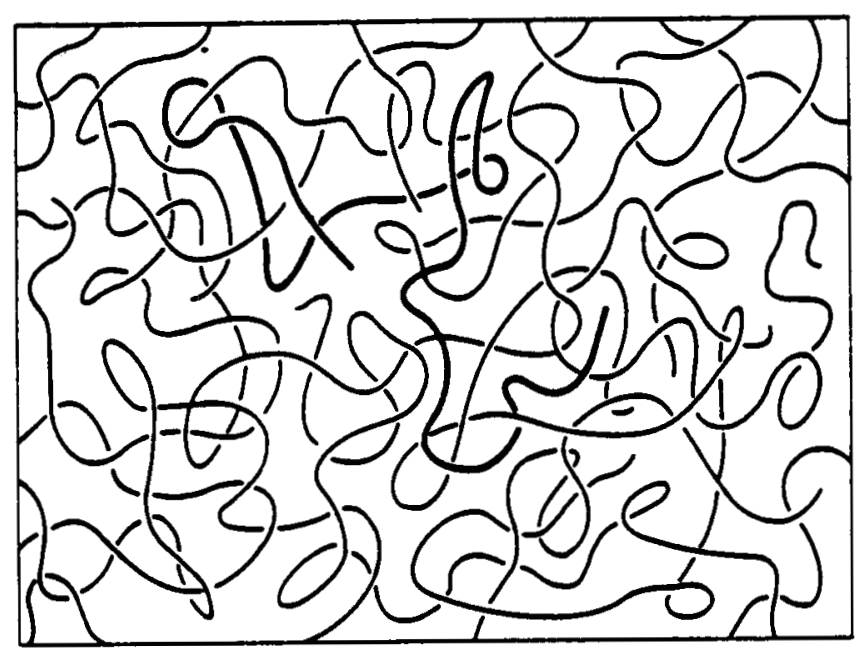

Figure 8. Random coil network for organic glasses. One polymer chain has been drawn with a thick line for ease of visualization.

of circumstellar silicates (Jones and Merrill 1976; Bedijn 1977; Schutte and Tielens 1985) may also indicate an amorphous structure (Tielens 1983). In this section the optical properties of amorphous materials is discussed with the emphasis on silicates. The optical properties of amorphous carbon grains are discussed by Allamandola, Tielens, and Barker elsewhere in this volume. Each of these three observational 
indications of the amorphous nature of interstellar grains will be discussed in turn.

\subsection{UV and Visible Absorption}

Absorption in the ultraviolet and visible spectral regions corresponds to changes in the electron structure of the material, which is dominated by the short-range order. In general, provided the chemical bonding is the same in crystalline and amorphous materials, the electronic density of states will also be very similar and therefore physical characteristics such as the energy gap will be about the same. Thus, the fundamental optical absorption edge in a crystalline and amorphous material will occur at a very similar frequency. For example, the sharp $r$ ise in the reflection spectra of amorphous and crystalline quartz at about $10 \mathrm{eV}$ arises from the onset of the bonding antibonding transitions of the electrons (Tauc 1972). Often, crystalline solids have special directions associated with the crystallographic axes, leading to anisotropy in the optical properties (i.e., dependence on crystal orientation). Amorphous materials lack such directionality, that is, they are isotropic.

Crystalline materials can show fine structure in their UV spectrum, particularly at low temperatures. Because each unit cell has an identical environment, such optical selectivity will arise because energy, as well as momentum, has to be conserved in the photon absorption process (cf. Kittell 1976). Such spectral structure is an intrinsic property of crystalline materials only, because it is a consequence of the longrange order (e.g., crystal symetry). It will be smoothed out in amorphous materials, because of the absence of periodicity (e.g., the wave vector is no longer a good quantum number). For example, crystalline materials show a rather abrupt onset of the electronic absorption edge, which in amorphous materials is much more gradual. Of course, spectral substructure in the UV can also result from the presence of more electronic transitions within the molecular sub-unit. Such substructure will be present in both crystalline and amorphous materials.

Silicates formed in the outflow from Miras show considerable absorption around $1 \mu \mathrm{m}$ (Jones and Merrill 1976; Bedijn 1977; Schutte and Tielens 1985). This high, near-infrared absorption is somewhat surprising since it occurs in the energy gap region; it may indicate an amorphous structure ( $T$ ielens 1983a). For an ideal crystalline dielectric material, there is an energy gap between the valence band and the conduction band. This energy difference is determined by the bondingantibonding splitting of the electronic states associated with the chemical bonding of the nearest neighbors. This energy gap is completely devoid of electronic states, and the crystalline material will be transparent in this region. An amorphous material will have 
localized electronic states (e.g., associated with a few atoms) in the energy gap region, and the density of electronic states is, thus, not zero but merely low. The transition probability of these states is, however, quite small and an amorphous material can be quite transparent (cf. glass). As a result, an amorphous material can still be character ized by an optical band gap energy. These localized states are therefore an unlikely source of the enhanced absorption of circumstellar silicates at about $1 \mathrm{\mu m}$; a more likely source is the presence of impurities (e.g., FeO) that can also produce localized electronic states in the energy gap region. Unfortunately, there are presently no laboratory studies that directly address this astrophysical problem.

\subsection{Mid-Infrared Absorption}

For low frequencies, the oscillations in the electromagnetic field are slow enough that molecular vibrations can respond to them, leading to absorption. Since the "force constants" between atoms and between atomds and electrons are very similar (i.e., both follow from Coulomb interaction), it follows from Hooke's law that while electronic trans1tions fall in the UV and visible, transitions involving atomic vibrations will occur in the infrared (i.e., $\left.\left(m_{e} / M\right)^{1 / 2}-10^{-3}\right)$. of course, the difference in mass also implies that the absorption strength of atomic vibrations is smaller by a factor of $10^{-3}$ (consider a harmonic oscillator) than that of electronic vibrations (e.g., $f_{I R}-10^{-2}-10^{-3}$ whereas $\left.f_{U V} \sim 1-10\right)$. Finally, it should be noted that damping in a solid is due to collisions with neighboring atoms. The collision rate is then approximately equal to the natural frequency of the vibration. The damping coefficient is proportional to this collision rate, to the anharmonicity of the interaction potential and, of course, to the number of phonons present (i.e., temperature).

In a crystalline solid the vibrational excitations are associated with plane waves, and they are therefore characterized by a frequency and a wave vector. These lattice vibrations are called phonons. Momentum conservation is more important for lattice vibrations than for electronic excitations, and only a small number of all the possible lattice vibrations are optically active. The infrared spectrum of a crystalline material consists, therefore, of a few sharp lines.

The disorder in an amorphous solid localizes the lattice vibrations and they can no longer be viewed as plane waves. As a consequence of the reduced symmetry, most modes will be infrared active and the infrared absorption spectrum reflects to a greater extent than it does in crystals the density of vibrational states. It should be emphasized that the vibrational density of states in an amorphous material is not much different from that of the corresponding crystalline material. Basically, it reflects the interaction between nearest neighbors, which 
is very similar in both types of materials. Of course, the increased dispersion in bond lengths and bond angles will produce a larger range in bond strength and thus in frequency (recall Hooke's law). Because of this and because most modes are IR active, the fundamental absorptinn features in an amorphous material will be broader than in a crystalline material.

The integrated strength of an absorption band in an amorphous material is generally somewhat less than that of the crystalline material. That the oscillator strength is very similar is of course not surprising, since the force constants of the oscillators are very similar. The larger disorder in an amorphous material implies, however, a larger damping coefficient (i.e., a larger effective coupling with phonons). As a consequence the peak strength of the absorption in an amorphous material is smaller and the width larger (cf. a damped oscillator). The absorption strength is related to the change in dipole moment. The high order in a crystalline material makes all dipoles change in unison, resulting in a fairly large change in dipole moment. In contrast, the disorder in an amorphous material will make the effective change in dipole moment less and consequently lower the integrated absorption strength (i.e., the oscillator strength). For silicates this difference in integrated absorption strength of amorphous and crystalline solids seems to be relatively large (a factor of 2.5 ; see $\$ 1.1$ ). This is much larger than in, for example, amorphous ice (25\%; Hagen et al. 1981). It should be noted, however, that the variation in peak absorption strength and width are much larger than the variations in the integrated strength. In general, it is therefore better to use the integrated strength in interpreting the strength of absorption features (cf. $\$ 1.1$ ).

Finally, it is interesting to consider the effects of temperature on the fundamental vibrations of a solid. Typically, absorption features sharpen and shift to slightly higher frequencies with decreasing temperature, but the integrated absorption strength stays approximately constant (Plendl 1970). These variations are a result of the thermal shrinking of the lattice. The decreased nearest neighbor distances imply larger force constants and thus higher frequencies (Hooke's law). The decrease in width is due to the decreased importance of anharmonicity and to a decrease in the number of phonons present, implying a smaller damping coefficient ( $c f$. a harmonic oscillator). Different modes (e.g., stretching versus bending vibrations) in a material may behave differently upon cooldown, since they may have different anharmonicity constants and they may interact with different phonons. This may account for the experimentally measured behavior of amorphous magnesium silicates (Day 1976).

At this point it is tempting to speculate that the decreased width of the $10 \mu \mathrm{m}$ feature in absorption in the interstellar medium 
(presumably at about $10-20 \mathrm{~K}$ ) as compared to the emission feature in Orion ( $\approx 250 \mathrm{~K}$; Gillett et al. 1975b; Roche and Aitken 1985) is due to the effects of thermal shrinking. However, no experimental data are available on the width of the $10 \mathrm{~mm}$ silicate band at low (10 K) temperatures. The variation seems, however, consistent with that measured for other materials (Plendl 1970).

\subsection{Far-Infrared Absorption}

The far-infrared $(\lambda>30 \mu \mathrm{m})$ absorption of amorphous materials differs considerably from that of crystalline materials. In crystalline materials most of the far-infrared absorption is due to multiphonon processes (for example, one photon and one phonon in, and one phonon of higher energy out). Fundamental vibrations (i.e., one photon in, one phonon out) are optically inactive because of wave-vector conservation (cf. \$3.2). Multiphonon processes are highly temperature sensitive, and at $10 \mathrm{~K}$ the absorptivity will be much reduced, essentially because there are few phonons around (Hadni 1970). Besides multiphonon processes, a material can also absorb in the damping wing of an infrared active fundamental vibration. Far from the natural frequency, the absorption coefficient of a damped harmonic oscillator will show a quadratic dependence with frequency, and such a dependence is expected for crystalline dielectric materials at low temperatures (Wooten 1972). In a metallic or semimetallic material (e.g., graphite), far-infrared absorption is due to interaction of the radiation with the free electrons (Wooten 1972). Again, the absorption coefficient will depend quadratically on the frequency, since the free electrons in a material are essentially "oscillators" without a restoring force. The damping is now due to scattering of the electrons by phonons (i.e., lattice vibrations).

In amorphous materials, the far infrared absorption is quite strong, often featureless, and not temperature sensitive (Hadni 1970). These differences with respect to crystalline materials are due to the loss of long-range order. The resulting breakdown of the selection rules implies that all modes are infrared active, and the far-infrared absorption spectrum reflects the density of vibrational states. Generally, the density of vibrational states is well described by the Debye law at low energies $\left(\sim \omega^{2} ;\right.$ Kittell 1976). The strength of the farinfrared absorption depends on the disorder in the charge distribution in the lattice. But, since the oscillator strength is independent of the particular vibrational mode involved in the phonon regions, the frequency dependence of the far-infrared absorption reflects directly the phonon spectrum (Tielens 1986a). Thus, an amorphous material will show the same wavelength dependence of the far-infrared absorption (i.e., $\lambda^{-2}$ ) as a crystalline material, albeit for completely different physical reasons. The far-infrared absorption of amorphous materials 
is, of course, much stronger and not temperature sensitive. The farinfrared spectrum of fused quartz shows these characteristics of amorphous materials (Hasegawa and Koike 1984).

An exception to the quadratic law for far-infrared absorption should be made for amorphous, layered materials, such as amorphous carbon, and layer-lattice silicates. The structure of such materials limits the phonons to two dimensions and, consequently, their phonon spectrum is proportional to the frequency rather than to the frequency squared (Kittell 1976). The temperature dependence of the specific heat of graphite, the classic example of a layered structure, reveals the presence of such a $\lambda^{-1}$ phonon spectrum (Touloukian and Buyco 1970). Because the far-infrared absorption of this material is dominated by free electrons, the far-infrared spectrum of this crystalline, layered material shows a $\lambda^{-2}$ dependence (see above). But, amorphous carbon, which has a very similar structure (cf. \$2.5) does show the expected $\lambda^{-1}$ far-infrared absorption law (Koike et al. 1980). Layer-lattice silicates, which also have a layered structure, show a $\lambda^{-n}$ farinfrared absorption law with $\mathrm{n}$ in the range 1.25 to 1.5 (Day 1976). Apparently, there is some degree of cross-linking between the layers in such materials, leading to a somewhat steeper law than expected for a layered structure (Tielens 1986a). The last case of a $\lambda^{-1}$ farinfrared absorption law involves small amorphous grains. In small particles (say (30 \&) the surface modes (two-dimensional) rather than the bulk modes (three-dimensional) will dominate the far-infrared absorption (Seki and Yamamoto 1980). Again, for amorphous materials where all modes are active, the two-dimensional structure will lead to a $\lambda^{-1}$ law.

Clearly, the far-infrared is a very useful tool for studying the structure of interstellar grains. Far-infrared observations of celestial objects generally show a far-infrared absorption law that is less steep than expected for crystalline materials (Campbell et al. 1976; Gatley et al. 1977; Harvey et al. 1978). This implies that most of the interstellar grains have an amorphous layered structure. Since amorphous carbon seems to be a relatively minor component of the interstellar dust (Table I), this suggests that interstellar silicates, which may make up half of the interstellar dust volume, have a layer-lattice amorphous structure. In this respect, it is worth noting that the $2.9 \mathrm{um}$ band observed in the spectrum of the galactic center can be well fitted by a spectrum of hydrated magnesium silicates, a layer-lattice silicate (Tielens et al. 1986 b). Obviously, a search for this band in other sources with strong silicate bands is important for the corroboration of this conclusion. 


\section{PHYSICAL PRINCIPLES OF GRAIN-SURFACE CHEMISTRY}

Figure 9 shows a schematic view of grain-surface chemistry. Atoms and molecules are accreted from the gas phase. They may diffuse and react on the surface, and the reaction products may remain on the surface, forming a grain mantle, or may actually be ejected from the surface by some means. In this section we will briefly discuss some of the physics of these processes. For a detailed discussion, the reader is referred to the tutorial by Watson (1976). This discussion will be geared toward $\mathrm{H}_{2}$ formation and the formation of icy grain mantles in molecular clouds.

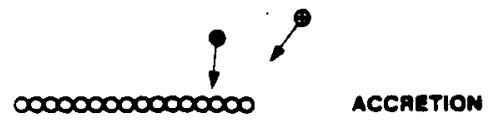

$\infty 000000000080000 \quad$ OIFFUSION

0000000080000000000 REACTION

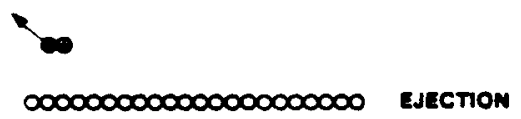

Figure 9. Grain-surface chemistry. Gas-phase species accrete, diffuse, and react on a grain surface. Possibly, the reaction product is ejected into the gas phase.

\subsection{Surface Structure}

The structure of the surface is of importance for many aspects of grainsurface chemistry. It should be realized that the structure of the surface will deviate from that of the bulk, in particular for a crystalline solid. In a sense, the surface is adjacent to a row of lattice vacancies, and as a result the equilibrium positions of the molecules at the surface will have been relaxed from that in the bulk. Moreover, the surface will facilitate the formation of lattice defects, which will further disturb the molecular bonds. Furthermore, the surface composition may differ from that of the bulk. Finally, a larger concentration of impurities may be present on the surface than in the bulk. Some of 
these points are illustrated in Figures 10 and 11, which show some of the structures possible at silicate and carbon surfaces.

(a) DEHYDRATION BELOW 700K

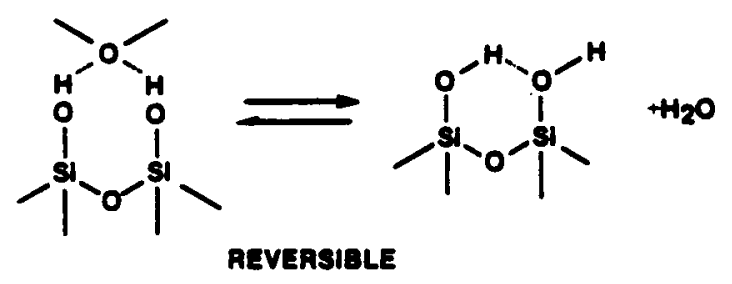

(b)

DEHYDAATION ABOVE 70OK

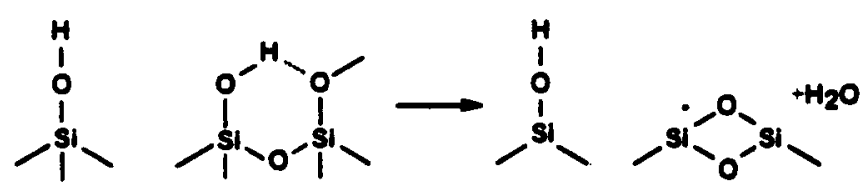

IAREVERSIBLE

Figure 10. Some of the structures possible at silicate surfaces. Note the presence of silanol ( $\mathrm{Si}-\mathrm{OH})$ and siloxane ( $\mathrm{Si}-\mathrm{O}-\mathrm{Si}$ ) groups. The former bonds $\mathrm{H}_{2} \mathrm{O}$ by hydrogen bonding.<smiles>Cc1cc2ccc(C)c(C)c2c(C)c1C</smiles>

HYDROGEN<smiles>Cc1cc2cc(C)c(C)c(C)c2c(C)c1C</smiles>

METHYL<smiles>Cc1c(C=O)cc2cc(N(C)C)c(C)c(C)c2c1C</smiles>

ALDEHYDIC<smiles>Cc1cc2cc(C(=O)O)c(C)c(C)c2c(C)c1C</smiles>

CARBOXYL

Figure 11. Some of the structures possible at the edge of the basal plane in graphite or amorphous carbon. 
Surface groups on silicates are extensively discussed in the monograph by Hair (1967) and by Snoeyink and Weber (1972). In an astrophysical context they have been briefly discussed by Duley and McCullough (1977). Generally, a silicate surface contains two types of surface groups, silanol groups $(\mathrm{S} i-\mathrm{OH})$ and siloxane groups $(\mathrm{Si}-\mathrm{O}-\mathrm{Si})$. Silicates precipitated from aqueous solutions at low temperature ( $<700 \mathrm{~K}$ ) mainly contain hydroxyl groups on the surface, a result of the tendency of the silicon atom to complete tetrahedral coordination. Water will be adsorbed on these surface silanol groups by van der Waals and hydrogen bonding forces. Upon heating to $700 \mathrm{~K}$, these physically adsorbed $\mathrm{H}_{2} \mathrm{O}$ molecules will be lost (dehydration of the silicate surface; Fig. 10). Adjacent hydroxyl groups will also form $\mathrm{H}_{2} \mathrm{O}$ molecules that are ejected from the surface, leaving a strained siloxane group behind. Because of the strain in these siloxane groups, rehydrogenation can occur, reforming the silanol groups. Warm-up above $700 \mathrm{~K}$ (or formation above this temperature) enables the strained siloxane groups to anneal themselves and, upon addition of water, no rehydrogenation will occur (e.g., the dehydrogenation is irreversible). Above $1000 \mathrm{~K}$, only isolated silanol groups (so-called free $\mathrm{OH}$ or terminal $\mathrm{OH}$ groups) and siloxane groups remain ( $\mathrm{Fig}$. 10). Such a surface will bind $\mathrm{H}_{2} \mathrm{O}$ much more weakly than the untreated silicates and is actually quite hydrophobic. The presence of other atoms in the silicate surface structure, such as Al, will increase the acidic nature of a silicate surface because of the inductive effect on adjacent silanol groups. Such atoms may also bind hydroxyl groups, which will behave slightly differently on heat treatment.

Physical adsorption of a polar molecule, such as $\mathrm{H}_{2} \mathrm{O}$, on a silicate surface will preferentially occur on the OH groups, and the heat of adsorption will increase with increasing polarity of the adsorbed molecule, a result of hydrogen bonding. The silanol groups on a silicate surface react as a weak acid and can, thus, react with bases (at room temperature). Reactions with alcohols (at high temperatures) will produce estersils (e.g., Si-O-C groups). At low temperatures, such reactions are inhibited by activation energies. Annealed siloxane groups are very weak bases and show, therefore, only a weak tendency to form hydrogen bonds. They are also quite unreactive. Because interstellar silicates condense out at relatively high temperatures ( $\sim 1000 \mathrm{~K})$, they are presumably initially quite hydrophobic. After processing in a shock they will presumably have many dangling surface bonds, which will be quickly saturated by $H$ atoms. The resulting surface may bind $\mathrm{H}_{2} \mathrm{O}$ more strongly. In any case, the low temperature of interstellar dust grains will inhibit reactions with surface molecules, and the only property of interest in interstellar grain surface chemistry is the ability of silicate surfaces to bond $\mathrm{H}_{2} \mathrm{O}$ and other polar molecules relatively strongly by hydrogen bonding. 
Commercial carbon blacks usually have several different surface groups besides aromatic hydrogen (e.g., methyl, phenol, and carboxyl groups; Fig. 11). These groups are substituted for the aromatic hydrogen at the edge of the aromatic ring system (Puri 197.6). They result from chemical reactions with the graphitic structure (at elevated cemperatures). Graphite or amorphous carbon freshly injected into the interstellar medium will contain mainly aromatic hydrogen at its edges because of the overabundance of hydrogen in their birth sites. Sputtering in fast shocks will change this surface structure and may lead to dangling bonds. These will either be resaturated by atomic hydrogen or the carbon atoms in the edge may revert to a divalent $s^{2} p^{2}$ state. The latter structure limits, of course, the resonance of the $\pi$ electrons and makes that site more reactive, but leaves no dangling bonds. In fact, vibrational spectroscopy of fluorescing interstellar PAHs, the building blocks of the carbon grains, generally shows an absence of surface groups other than aromatic hydrogen (Allamandola, Tielens, and Barker, elsewhere in this volume), and this is likely to be true for carbon grains as well.

\subsection{Adsorption Energies}

A species can be either physically or chemically bonded to the surface. In physical adsorption the interaction is due to van der WaalsLondon or electric field-dipole forces, and the interaction energy is fairly low $(<0.5 \mathrm{eV})$. There is no charge exchange between the adsorbed species and the substrate in physical adsorption. The attractive force is due solely to the interaction of the (instantaneous) dipole of the adsorbed species and the neighboring surface molecules (Kittell 1976). In chemisorption, the interaction leads to actual chemical bond formation and the interaction energy is much larger $(-0.5-5 \mathrm{eV})$. This interaction can entail a complete charge exchange between the surface and the adatom and the formation of an ionic bond. Usually, however, the electronic wave functions of the adatom and the surface mix into a new wave function. Essentially, the electrons responsible for the bonding move in orbitals between the surface and the adatom, and a covalent bond is formed.

The theoretical description of chemisorption is very complex and not completely understood (cf. Einstein et al. 1980). For illustration purposes consider the physical processes occurring when an adatom approaches a metal surface. A crude impression of the tendency to form a chemical bond can be obtained by comparing the adsorbate's ionization potential and electron affinity with the work function of the surface. If the ionization potential of the adatom is less than the work function of the surface, then an electron can be transferred to the surface and the adatom becomes positively charged. Charge transfer can also take 
place when the work function is less than the electron affinity of the adatom. Now the electron is transferred to the adatom. In both cases, chemisorption is energetically feasible. However, if the work function of the surface is larger than the electron affinity but less than the ionization potential of the adatom, then the adatom will be physically adsorbed. This discussion is of course very crude because the interaction between an approaching atom and a surface will lead to broadening of the atomic states, and the ionization and affinity levels will become bands whose widths depend on the degree of interaction (cf. Einstein et al. 1980). These bands may partially overlap with the (Eermi) level of the metal. When the atom is very close to the surface, strong inter action may actually lead to a complete modification of the electronic structure of the atom and the surface.

A simple potential energy diagram for chemisorption is shown in Figure 12. Note that the physical adsorption forces have a longer range than the chemical forces and, thus, an accreting species will first encounter the physical adsorption potential. For atoms approaching a surface there is no activation barrier associated with chemisorption. For molecules, however, there is probably an activation barrier because a molecular bond has to be weakened or broken. In the interstellar medium, chemisorption of molecules is, therefore, generally unimportant owing to the low temperatures.
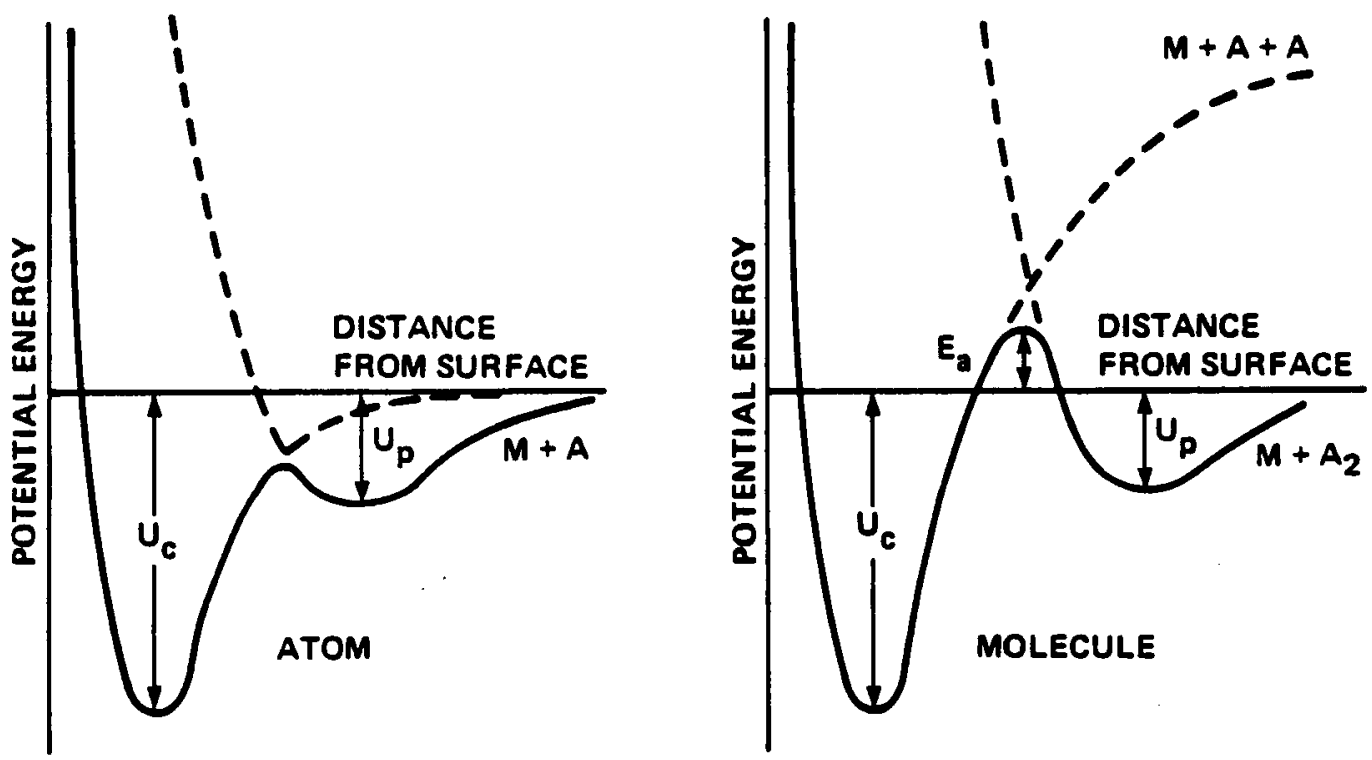

Figure 12. A potential energy diagram for the adsorption of an atom (a) or a molecule (b) on a surface. Note that there are two adsorption wells, the physical adsorption well with binding energy, $U_{p}$ and the chemisorption well with binding energy, $U_{c}$. For molecules, these wells may be separated by an activation energy, $E$. 
Hydrogen atoms on silicate surfaces have two types of binding sites. First, a chemisorbed site with a binding energy of about $2 \mathrm{eV}$ $\left(-2.3 \times 10^{4} \mathrm{~K}\right)$. Second, a physical adsorbed site with a binding energy of only about $0.09 \mathrm{eV}$ ( $-1000 \mathrm{~K}$; Gelb and Kim 1971; Wood and Wise 1962; Hickmott 1960; de Boer and van Steenis 1952; Langmuir 1912, 1915). The second sites should be viewed to be on top of the first one ( $c$ f. Fig. 12) and will only be occupied when the first one is already filled. Note that reaction between $H$ atoms in the two layers is inhibited by an activation barrier $(-1000 \mathrm{~K})$ associated with breaking the chemisorbed bond with the surface (see $\$ 5$ ). The number of sites on a silicate surface is about $1 \times 10^{15}$ to $2 \times 10^{15} / \mathrm{cm}^{2}$, corresponding to about $7 \AA^{2}$ per site.

Although it is well known that atomic hydrogen can be chemisorbed on the basal plane of graphite as well as on the edges (King and Wise 1963; Wood and Wise 1969; Rye 1977; Gould 1975; Balooch and Olander 1975; Robell et al. 1964), the parameters of these states are less well known, a result of the complicated chemistry of $\mathrm{H}$ atoms with the edge structure of such surfaces at elevated temperatures $(300 \mathrm{~K})$. At high surface coverage, $\mathrm{H}_{2}$ formation has an activation barrier of about $1200 \mathrm{~K}$, suggesting an $H$ chemisorption energy on the basal plane of perhaps $2 \mathrm{eV}$. From diffusion experiments, McCarroll and McKee (1971) estimated a somewhat lower binding energy of about $0.9 \mathrm{eV}$ on the basal plane. For $\mathrm{H}_{2}$ formation on interstellar grains, the activation energy is, however, more important than the binding energy (see \$5), so that this discrepancy should not worry us too much. The "aromatic" $\mathrm{H}$ atoms at the edge of the basal plane are chemically bonded by about $5 \mathrm{eV}$. The physical adsorption energy of $H$ atoms on the basal plane of graphite is measured to be about $800 \mathrm{~K}$ (Lee 1975). The number of chemisorption sites is again of the order of $10^{15} \mathrm{~cm}^{-2}$. Other atoms on silicate and graphite surfaces are also expected to be chemisorbed on these sites. At low temperatures, molecules will be physically adsorbed on these surfaces.

Atoms and molecules will be physically adsorbed on icy grain. Their binding energies can be determined from molecular beam studies and from diffusion experiments in low-temperature matrices. Table II sumarizes estimates for the binding energy of relevant atoms and molecules on an icy surface.

Using these binding energies, the residence time of a species on a surface can be calculated

$$
\tau=\tau_{0} \exp \left(E_{b} / k T\right) \text {, }
$$

where $\tau_{0}$ is the oscillation time of the adsorbed species perpendicular to the surface (Frenkel 1924). Essentially, the adsorbed molecule has replaced at least one degree of translationary freedom by a vibrational 
TABLE 2

The Interaction of Atoms and Molecules with an $\mathrm{H}_{2} \mathrm{O}$ Surface

\begin{tabular}{|c|c|c|c|c|c|c|c|}
\hline \multirow[t]{2}{*}{ Species } & \multirow[t]{2}{*}{$E_{b}(K)^{1}$} & \multicolumn{2}{|c|}{$\tau_{e v}(\sec )^{2}$} & \multicolumn{3}{|c|}{$\tau_{m}(\sec )^{3}$} & \multirow[t]{2}{*}{ Notes } \\
\hline & & 10 & 30 & 5 & 10 & 30 & \\
\hline $\mathrm{H}$ & 350 & $1.6 \times 10^{3}$ & $10^{-7}$ & $10^{-12}$ & $10^{-12}$ & $10^{-12}$ & 4 \\
\hline $\mathrm{H}_{2}$ & 450 & $3 \times 10^{7}$ & $3 \times 10^{-6}$ & $4 \times 10^{-12}$ & $4 \times 10^{-12}$ & $4 \times 10^{-12}$ & 4 \\
\hline$c$ & 800 & & $4 \times 10^{-1}$ & $10^{8}$ & $10^{-2}$ & $2 \times 10^{-9}$ & 5,6 \\
\hline$N$ & 800 & & $4 \times 10^{-1}$ & $10^{8}$ & $10^{-2}$ & $2 \times 10^{-9}$ & 5,6 \\
\hline 0 & 800 & & $4 \times 10^{-1}$ & $10^{8}$ & $10^{-2}$ & $2 \times 10^{-9}$ & 5,6 \\
\hline$S$ & 1100 & & $8 \times 10^{13}$ & & $10^{2}$ & $4 \times 10^{-8}$ & 6,7 \\
\hline $\mathrm{CO}$ & 1900 & & & & $6 \times 10^{12}$ & $2 \times 10^{-4}$ & 5,8 \\
\hline $\mathrm{N}_{2}$ & 1700 & & & & $10^{10}$ & $2 \times 10^{-5}$ & 5,8 \\
\hline $\mathrm{O}_{2}$ & 1600 & & & & $7 \times 10^{8}$ & $9 \times 10^{-6}$ & 5,8 \\
\hline $\mathrm{CH}_{4}$ & 2600 & & & & & $2 \times 10^{-1}$ & 5,8 \\
\hline $\mathrm{H}_{2} \mathrm{O}$ & 4000 & & & & & $2 \times 10^{5}$ & 9,6 \\
\hline
\end{tabular}

Notes: 1) Estimated binding energy of a species on an $\mathrm{H}_{2} \mathrm{O}$ surface.

2) Evaporation time-scale from an $\mathrm{H}_{2} \mathrm{O}$ surface for grain temperatures of 10 and $30 \mathrm{~K}$. No value is given when it exceeds the lifetime of a molecular cloud.

3) Migration time-scale on an $\mathrm{H}_{2} \mathrm{O}$ surface for grain temperatures of 5,10 and $30 \mathrm{~K}$. No value is given when it exceeds the lifetime of a molecular cloud.

4) Binding energy determined from experimentally measured attractive potential corrected for zero point energy and $\mathrm{H}_{2}$ coverage (see text).

5) Binding energy determined from measured maximum attractive potential for $\mathrm{H}_{2}$, corrected for difference in polarizability (cf. Watson 1976).

6) Diffusion barrier determined from low temperature matrix isolation studies ( $T$ ielens and Hagen, unpublished).

7) Binding energy determined from diffusion experiments in Low temperature matrices in analogy to oxygen.

8) The diffusion barrier is assumed to be $30 \%$ of the binding energy. $\mathrm{H}_{2} \mathrm{O}$ surface.

9) Enhanced binding energy due to hydrogen bonding with the 
degree of freedom of the bond with the surface. It may also have lost some degrees of rotational freedom. For a monoatomic gas which is completely mobile on the surface, we can find for $\tau_{0}$ from a statistical thermodynamical analysis

$$
\tau_{0}=(h / k T) f_{z},
$$

where $f_{z}$ is the vibrational partition function of the adsorbed molecule (de Boer 1968). At high temperatures the vibration perpendicular to the surface is excited and $f_{z}$ is equal to $\mathrm{kT} / \mathrm{h} v_{z}$, where $\nu_{z}$ is the vibrational frequency perpendicular to the surface and $\tau=v^{-1}$.

We will assume that the frequency of vibration perpendicular to the surface is equal to that parallel to the surface, which for a symmetric harmonic potential is given by (Landau and Lifshitz 1960):

$$
v_{2}=\left[\left(2 N_{s} E_{b}\right) / \pi^{2} m\right]^{1 / 2},
$$

where $N_{s}$ is the number of sites per unit surface area and $m$ the mass of the atom. With a physical adsorption energy of $1000 \mathrm{~K}$ and $N_{s}=\left(7 \AA^{2}\right)^{-1}, v_{z}$ is about $5 \times 10^{12} \mathrm{sec}^{-1}$ for an $H$ atom and about $10^{12} \mathrm{sec}^{-1}$ for an 0 atom. For a chemisorbed site with a binding energy of $2 \mathrm{eV}, v_{z}$ is about $2 \times 10^{13}$ and $5^{\circ} \times 10^{12} \mathrm{sec}^{-1}$, respectively. At low temperatures, the vibration perpendicular to the surface is not excited and $f_{2}=1$, leading to $\tau_{0}=h / k T=5 \times 10^{-12} \mathrm{sec}$, where a temperature of $10 \mathrm{~K}$ has been assumed. When the adsorbed species is not mobile on the surface or has lost some rotational degrees of freedom, then equation (5) has to be multiplied by the ratio of the partition functions for hindered and free translationary motion on the surface or the rotational partition functions on the surface and in the gas phase, respectively. In both these cases $\tau_{0}$ will be less than $h / k T$. In the limit that an adsorbed atom has lost all its degrees of translationary freedom, a different expression is derived:

$$
\tau_{0}=(h / k T) f_{x} f_{y} f_{z}\left(N_{s} h^{2} / 2 \pi m k T\right)
$$

where the $f_{x}, f_{y}$ are the partition functions along the surface (de Boer 1968). The last factor in this expression takes into account the number of possible ways of distributing the adsorbed species over the adsorption sites. Consider again the low-temperature limit $(T=10 \mathrm{~K})$, assume a surface area per site of $7 \AA^{2}$, and a mass of 20 amu. This yields. $\tau_{0}=4 \times 10^{-12} \mathrm{sec}$. Thus, for the interstellar case, $\tau_{0}$ lies between $2 \times 10^{-13}$ and $4 \times 10^{-12}$ sec for an $H$ atom physically adsorbed and chemisorbed, respectively. Using $\tau_{0}=10^{-12} \mathrm{sec}$, we find the residence time-scales on an icy surface given in Table II. 


\subsection{Sticking Probabilities}

A gas-phase species approaching a grain experiences an attractive force owing to the molecules in the grain. Whether a gas-phase species will stick or not depends on two factors: (1) the magnitude of its translational energy compared to the adsorption energy and (2) the efficiency with which the excess kinetic energy can be transferred to the surface atoms with which it is colliding. Some insight into the physics can be gained by examining the classical case. Imagine an atom with mass $m$ colliding with a single surface atom of mass $M$ which is attached to the surface with a spring of a frequency. $\omega_{D}$ (the Debye frequency). This is the so-called "soft-cube" model. We can define a characteristic inverse time-scale for the collision, $\omega_{0}$,

$$
\omega_{0}=v / 2 b=\left[\left(E_{t h}+E_{b}\right) / 2 m b^{2}\right]^{1 / 2}
$$

where $v$ is the velocity during the approach, $b$ is a characteristic length scale for the repulsive interaction $(\approx 0.3-0.4 \AA)$, and $E_{t h}$ and $E_{b}$ are the kinetic and binding energies, respectively. For an $H$ atom at low temperatures, $\omega_{0}$ is typically $3 \times 10^{14}$ and $6 \times 10^{13} \mathrm{sec}^{-1}$ for chemisorption and physical adsorption, respectively. When the collision time is short compared to the spring period (i.e., $\dot{\omega}_{0} \gg \omega_{D}$ ) then the energy transferred in the collision is

$$
\Delta E=4(m / M)\left(E_{t h}+E_{b}\right)
$$

(Hollenbach and Salpeter 1970). This transferred energy should be compared to the initial kinetic energy of the impacting atom, $E_{t h}$, to determine whether sticking will occur. If the collision time-scale and the spring period are equal (i.e., $\omega_{0}=\omega_{D}$ ) then the energy transferred in the collision is one fourth that given by equation (9). If $\omega_{0}$ is actually much less than the spring frequency, then other surface atoms can couple in the collision. The energy transferred is then decreased over that given by equation ( 9 ) by a factor of about $1 / 2\left(\omega_{0} / \omega_{D}\right)^{3}$ (Hollenbach and Salpeter 1970; Watson 1976). Consider, for example, an $\mathrm{H}_{2}$ molecule with a kinetic energy corresponding to $300 \mathrm{~K}$ colliding with an ice surface $\left(E_{b} \approx 800 \mathrm{~K}\right)$. The maximum energy transfer is $50 \%$ of the total energy (cf. eq. [9]) or somewhat less than $600 \mathrm{~K}$. Actually $\omega_{D}$ is about equal to $\omega_{0}$ in this case, and the energy transferred is only about $140 \mathrm{~K}$, leaving about $160 \mathrm{~K}$ of kinetic energy, and sticking will not occur in the initial collision. Because the collision is generally not head-on, the approaching atom. will, however, ricochet off and collide with other surface atoms before being finally ejected from the surface; therefore, more energy can be transferred (Hollenbach and Salpeter 1970). Taking this into account, the sticking coefficient of 
an $\mathrm{H}_{2}$ molecule on an $\mathrm{H}_{2} \mathrm{O}$ surface at $300 \mathrm{~K}$ is calculated to be about 0.3 (Hollenbach and Salpeter 1970), close to the experimental value (Govers et al. 1980). Of course when the surface is covered with a layer of $\mathrm{H}_{2}$ molecules, as is likely inside molecular clouds, then the sticking coefficient will increase to about unity (i.e., $\Delta E=E_{\text {th }}+E_{b}$ ), in agreement with experiments (Govers et al. 1980).

In general, if the kinetic energy of the accreting atom is much less than the adsorption energy, then only a relatively small amount of energy needs to be transferred to the surface, and the sticking coefficient will be relatively large. Similarly, if the accreting atom is much heavier than the surface atoms, then the amount of kinetic energy transferred to the surface will be relatively large and the sticking coefficient will also be relatively large. Finally, for a stiffer lattice (e.g., $\omega_{D} \gg \omega_{0}$ ) the sticking coefficient will be lower than for a softer lattice. It should be noted that this classical description assumes that any amount of energy can be transferred in the collision and, thus, when the initial kinetic energy goes to zero, the sticking coefficient will go to unity. This is, however, not correct, and for low initial energies quantum effects have to be taken into account ( $c$. Leitch-Devlin and Williams 1985). The adatom has to wind up in a bound level in the interaction potential well, and, thus, a minimum amount of energy has to be transferred, which depends on the spacing of the upper levels in the potential well. Because of anharmonicity this will be of little importance for chemisorption, but it can be of importance for physical adsorption. It should also be kept in mind that the phonon spectrum of a solid degreases with energy $(\mathrm{e} . \mathrm{g}$. , the phonon density is proportional to $\left(\omega / \omega_{D}\right)^{2}$, so that coupling and thus energy transfer to the lattice will be less at low impact energies. Again, this is mainly important for physical adsorption for which the interaction potential is quite small.

The sticking coefficients can be found from the theoretical and experimental results. Anticipating our discussion later on, the sticking probabilities of importance for interstellar grain chemistry are the collision of a species with a clean silicate or graphite surface on which it can be chemisorbed (e.g., in the diffuse interstellar medium) and the collision of a species with an icy grain mantle on which it will be physically adsorbed (e.g., inside dense molecular clouds). Theoretical calculations on the sticking probability of an atom on a grain surface have been evaluated for several likely gas-phase-species and grain combinations (Hollenbach and Salpeter 1970; Watson and Salpeter 1972; Burke and Hollenbach 1984; Leitch-Devlin and Williams 1985). These calculations show that an $\mathrm{H}$ atom colliding with a silicate or graphite surface on which it can be chemisorbed will have a sticking coefficient of unity at a gas temperature of $100 \mathrm{~K}$. An $\mathrm{H}$ atom colliding with a clean $\mathrm{H}_{2} \mathrm{O}$ ice surface at $10 \mathrm{~K}$ has a relatively low sticking 
coefficient $(\approx 0.3)$. However, inside a molecular cloud the icy surface will be partially covered with $\mathrm{H}_{2}$ molecules, and this will raise the sticking coefficient to close to unity. For heavier atoms, the larger binding energy and larger mass will insure a large sticking probability $(\approx 1)$.

An exception to these high sticking probabilities may have to be made for ions accreting on charged grains (Watson and Salpeter 1972). Grains inside molecular clouds are negatively charged most of the time because the electrons have a greater velocity than the ions (Spitzer 1978). Charge recombination at or near the surface complicates the evaluation of the sticking coefficient considerably (Watson and Salpeter 1972). The Coulomb interaction energy is much larger than the adsorption energy of neutrals on icy grains considered above (i.e., comparable to a chemisorption energy). Thus, an accreting ion acquires a much larger approach velocity than a neutral. The Coulomb interaction may lead to the tunneling of an electron through the work function of the grain material, followed by ion-electron recombination, possibly before the ion has reached the surface. The resulting neutral experiences the much weaker, neutral-grain adsorption potential, but its kinetic energy is still that of the original ion. This is, therefore, a case in which the kinetic energy of the gas-phase species is much larger than the adsorption energy and, consequently, the sticking probability of ions will be very low (Watson and Salpeter 1972). This hinges, however, on the mobility of electrons in grains, and if electrons are actually quite localized in a grain then the ion-sticking probability will be close to unity. Of course, the Coulomb interaction will also enhance the collision cross section by about an order of magnitude (i.e., focusing).

A parameter of importance for molecule formation on grain surfaces is the time-scale at which a grain accretes species from the gas phase. This accretion time-scale is given by

$$
\tau_{a c}=\left(n_{i} \sigma v S\right)^{-1} \text {, }
$$

where $n_{i}$ is the density of the species in the gas phase $\left(5 \times 10^{-4} n_{0}\right)$, $\sigma$ is the geometrical cross section of the grain $\left(10^{-9} \mathrm{~cm}^{2}\right), v$ the average velocity $(1 \mathrm{~km} / \mathrm{sec})$, and $S$ the sticking probability $(\approx 1)$. thus, inside dense molecular clouds a grain will see a very small flux of incoming atoms or molecules (e.8., a few per day at a density of $\left.10^{4} \mathrm{~cm}^{-3}\right)$. Of course, with this flux all the heavier species in the gas phase will have accreted in $10^{5} \mathrm{yr}$, which is a much shorter period than the cloud's lifetime. 
The binding energy of an adsorbed species varles considerably across tre surface owing to the discrete nature of the lattice. For physical adsorption, the barrier against diffusion to an adjacent site, $E_{d}$, is typically about $30 \%$ of the binding energy, although sometimes much lower values have been calculated (Jaycock and Parfitt 1986). For chemisorption these variations can be comparable to the binding energy, although often it is again about half the binding energy. For a heavy species the migration time-scale, $\tau_{m}$ (i.e., the time to go to a neighboring site), is given by the Boltzman expression:

$$
\tau=v_{z}^{-1} \exp \left(E_{d} / k T\right) \text {. }
$$

Here $v_{z}$ is the frequency of the vibrational motion along the surface (cf. eq. [6]). For atomic hydrogen, deuterium, and helium and molecular hydrogen, migration is due to quantum mechanical tunneling. The timescale for this process is given by

$$
\tau_{m}=4 h / \Delta E,
$$

where $\dot{\Delta E}$ is the width of the lowest energy band (cf. Hollenbach and Salpeter 1970; Watson 1976). For atomic $\mathrm{H}$ on an $\mathrm{H}_{2} \mathrm{O}$ surface this has been evaluated to be $30 \mathrm{~K}$, yielding a surface diffusion time-scale of $10^{-12} \mathrm{sec}$. Values for the migration time-scale on an icy surface are given in Table II. It should be stressed that the actual time involved in moving from one site to a neighboring site is very small $\left(=10^{-12} \mathrm{sec}\right)$. The limiting step in the migration process is the acquisition of the energy required to overcome the diffusion barrier.

The surface diffusion process is essentially a random-walk one. The rms value of the displacement vector $R$, after a time $\Delta t$ is then

$$
\left\langle\mathrm{R}^{2}\right\rangle^{1 / 2}=\mathrm{N}^{1 / 2} \ell
$$

where $l$ is the length of one site and $N$ the number of hops during this time $\left(N=\Delta t / \tau_{m}\right)$. Thus, the average time to scan a grain surface, $\tau_{s}$, is $\tau_{s}=A_{s} \tau_{m} / 4 \pi$, where $A_{s}$ is the total number of sites on the surface ( $\sim 10^{6}$ sor a $1000 \AA$ grain).

\subsection{Surface Reactions}

Here we will only consider the formation of icy grain mantles inside dense clouds. The reactions associated with $\mathrm{H}_{2}$ formation are discussed in $\$ 5$. The reaction network can be limited considerably by a comparison of the migration time-scale and the thermal evaporation time-scale 
(Table II) with the accretion time-scale (eq. [10]; $\sim 10^{5} \mathrm{sec}$ ). Species with a migration time-scale much less than the accretion time-scale can diffuse on the surface until they find a co-reactant $\left(\mathrm{e} . \mathrm{B} ., \mathrm{H}, \mathrm{H}_{2}, \mathrm{C}, \mathrm{N}\right.$, 0 , $S$ at $10 \mathrm{~K})$. Otherwise they are trapped in their site and can only react with migrating co-reactants that visit their sites $\left(e .8 ., \mathrm{CO}, \mathrm{N}_{2}\right.$, $\mathrm{O}_{2}, \mathrm{CH}_{4}, \mathrm{NH}_{3}$, and $\mathrm{H}_{2} \mathrm{O}$ at $\left.10 \mathrm{~K}\right)$. Thus, at $10 \mathrm{~K}$ the grain mantle reaction network contains reactions of the migrating atoms $H, C, N$, and 0 among themselves and with the non-diffusing atoms or radicals (Tielens and Hagen 1982).

Comparison of the evaporation time-scale with the accretion timescale shows that if atomic $H$ does not find a reaction partner on the surface then it will evaporate before the next radical accretes. Direct recombination of two $\mathrm{H}$ atoms to form $\mathrm{H}_{2}$ does not occur on icy grain surfaces in molecular clouds (of. \$5). It should be stressed that because of their low mass, $H$ atoms can tunnel through activation barriers and react with molecules containing an even number of electrons (e.g., non-radicals), such as $\mathrm{CO}, \mathrm{O}_{2}, \mathrm{H}_{2} \mathrm{CO}, \mathrm{H}_{2} \mathrm{O}_{2}, \mathrm{H}_{2} \mathrm{~S}$, and $\mathrm{O}_{3}$ (Tielens and Hagen 1982). The time-scale for tunneling through a barrier with a height, $E_{a}$, and a width, $a$, is given by

$$
\tau_{t}=\tau_{0} \exp \left[(2 a / h)\left(2 m E_{a}\right)^{1 / 2}\right]
$$

where $m$ is the mass of the tunneling species. Assuming a barfier height of $2000 \mathrm{~K}$ and a width of $1 \AA$, this time-scale is $2 \times 10^{-4} \mathrm{sec}$. This is much longer than the migration time-scale, and the probability for reaction is therefore very small $\left(\tau^{\prime} / \tau_{t} * 5 \times 10^{-9}\right)$. Thus, generally an $H$ atom will migrate to an adjacent site rather than react. However, over an evaporation time-scale, an $H$ atom will visit each site many times ( $\tau_{\mathrm{ev}} / \tau_{\mathrm{s}}=10^{10}$ at $10 \mathrm{~K}$ for a $1000 \AA$ grain). The probability of reaction $\mathrm{P}_{r}$ is therefore given by

$$
p_{r}=\left(\tau_{e v} / \tau_{s}\right)\left(\tau_{m} / \tau_{t}\right) \cdots
$$

For the numerical example considered above, $\mathrm{Pr}_{\mathrm{r}}$ is about 5 . Thus, $H$ atoms can react with molecules even if there is an activation barrier as large as $2000 \mathrm{~K}$. For a high concentration of co-reactants, $\mathrm{pr}_{r}$ will be larger. For example, for a concentration of $1 \%$ on a grain of radius $10^{3} \AA$, the limiting barrier an $H$ atom can tunnel through is about $3500 \mathrm{~K}$. Similarly, reactions of $\mathrm{H}_{2}$ with an activation barrier less than about $4500 \mathrm{~K}$ can be important on grain surfaces (Tielens and Hagen 1982). Heavier atoms or radicals will have to thermally hop over an activation barrier and reactions. with activation barriers larger than the barrier against diffusion are effectively inhibited unless the abundance of co-reactants is very large. Assuming the surface is covered with such co-reactants and considering the accretion of a new 
radical as the limiting step for reactions of 0 atoms frather than evaporation), reactions of 0 atoms with an activation energy of about $450 \mathrm{~K}$ or less have to be taken into account at $10 \mathrm{~K}$. Reactions of 0 atoms with negligible activation barriers include $\mathrm{O}_{2}$ and possibly $\mathrm{CO}$ forming $\mathrm{O}_{3}$ and $\mathrm{CO}_{2}$, although the latter activation barrier is still somewhat controversial ( $T$ ielens et al. 1986a).

\subsection{Dissipation of Reaction Heat}

The product molecules of grain-surface reactions are generally formed in electronically and vibrationally excited states. The subsequent decay, ultimately to the ground state, stabilizes the newly formed molecule. Some of the reaction heat might be transferred into center-of-mass translational motion, possibly leading to ejection of the molecule. The excess translational energy of the ejected molecule will heat the gas. The ejected molecule may also contain excess vibrational or rotational energy, which will decay radiatively. This process might explain the relatively high excitation of $\mathrm{J}>4$ levels of $\mathrm{H}_{2}$ observed in diffuse interstellar clouds, although other mechanisms are also possible (Spitzer and Cochran 1973; Dalgarno 1975).

In particular, the ejection of "hot" $\mathrm{H}_{2}$ from grain surfaces has incited several theoretical studies. Classical mechanical trajectory calculations have been performed, mainly for $\mathrm{H}_{2}$ formed by recombination of two physically adsorbed $H$ atoms on a grain surface (Hollenbach and Salpeter 1970; Hunter and Watson 1978). In these calculations enough energy was transferred to the center-of-mass translational motion in the collision between the newly formed $\mathrm{H}_{2}$ molecule and the surface potential to cause ejection. Similar calculations for heavier molecules indicate that their ejection is unlikely (Watson and Salpeter 1972). Essentially the coupling between vibrations and center-of-mass translational motion is small, because the molecular vibrational time-scale is much shorter than that for change in the repulsive interaction potential with the'. surface (Hunter and Watson 1978). However, these calculations suffer from two deficiencies: they ignore transfer of energy to other decay channels than center-of-mass translational motion and the quantum nature of the energy transfer process. It should be noted that molecule formation may occur along a pathway that is directly dissociative in the surface-admolecule bond (Aronowitz and Chang 1985). The general importance of this ejection mechanism is, however, unknown, and we will ignore this channel in the subsequent discussion.

The transfer of the reaction heat to other channels is very important. In fact, in the classical trajectory calculations referred to above, a somewhat arbitrary criterion had to be used to determine the fraction of $\mathrm{H}_{2}$ molecules ejected, because in the absence of other decay channels all newly formed $\mathrm{H}_{2}$ molecules will eventually acquire enough 
energy in their center-of-mass translational motion to be efected. Among the other energy channels that can accept the excess vibrational energy of a newly formed $\mathrm{H}_{2}$ molecule are rotations of the $\mathrm{H}_{2}$ molecule (rapidly followed by transfer to lattice phonons), (de)localized phonons of the grain, and vibrational modes of neighboring molecules. Because of the inherent quantum nature of the transfer process, the contribution of each of these processes depends on the spacing of the energy levels involved, as well as on the nature of the interaction.

Except for energy transfer to neighboring molecules, the accepting energy channel has a much smaller energy level spacing than the initial vibrational energy channel. For example, a vibrational quantum of $\mathrm{H}_{2}$ is 3000 to $5000 \mathrm{~cm}^{-1}(\approx 0.4-0.67 \mathrm{eV})$, which is much larger than an $\mathrm{H}_{2}$ rotational quantum $\left(\approx 120 \mathrm{~cm}^{-1} ; \approx 0.015 \mathrm{eV}\right)$, a vibrational quantum of the $\mathrm{H}_{2}$-surface bond $\left(=30 \mathrm{~cm}^{-1} ; \approx 0.004 \mathrm{eV}\right)$, or phonons $\left(\approx 100 \mathrm{~cm}^{-1}\right.$; $\approx 0.01 \mathrm{eV}$ ). Therefore, in the energy-transfer process a large number of quanta have.to be created simultaneously. This process is described by an energy-gap law, and its rate decreases exponentially with the energy gap involved, that is, with the number of quanta that have to be generated (Diestler 1974; Nitzan et al. 1975; Diestler et al. 1977). Experimental studies of the energy transfer from vibrational $\left(\approx 3000 \mathrm{~cm}^{-1}\right)$ to rotational $\left(=30 \mathrm{~cm}^{-1}\right)$ modes of small hydrides isolated in lowtemperature matrices indeed show such an energy-gap law (Legay 1977).

Energy transfer to neighboring molecules can be a very important energy decay channel for vibrationally excited molecules if the energy mismatch between the donor and acceptor is not too large and if the exchange is symmetry allowed. This energy exchange is induced by (longrange) multipolar (i.e., dipole or quadrupole) interactions. Theoretically, the lifetime associated with this energy decay channel is estimated to be about $5 \times 10^{-12} \mathrm{sec}$ for resonant conditions (Brus 1981). Experimental studies of resonant energy transfer of vibrationally excited $C O$ indicate a lifetime of about $10^{-11} \mathrm{sec}$ (Legay 1977). Energy transfer in the Lorentzian wing of a transition is very inefficient and non-resonant energy transfer is, therefore, generally assisted by phonons (or other low-lying energy channels that match). This will again lead to an exponential decrease in the rate with increasing energy gap. This is confirmed by experimental studies (Legay 1977). Nonresonant energy transfer can still be very fast, if the conditions are favorable.

As an example of these principles consider the different energydecay channels for the excess vibrational energy of a newly formed $\mathrm{H}_{2}$ molecule one by one. Since this is a homonuclear diatomic molecule, it does not possess a dipole moment. Near-resonant quadrupole-dipole transfer to neighboring molecules of the excitation in the high vibrational levels $\left(v-3000 \mathrm{~cm}^{-1}\right.$ ) therefore is probably slower by a factor of $10^{2}$ to $10^{3}$ than the 10 psec time-scale for dipole-dipole interaction, 
leading to a time-scale of perhaps $10^{-9}$ to $10^{-8} \mathrm{sec}$. It should be noted that interstellar grain surfaces are expected to contain many functional groups with vibrational modes at about $3 \mathrm{\mu m}$ (e.8., $\mathrm{OH}$ and $\mathrm{CH}$ groups; cf. \$4.1), which can act as energy acceptors. Recall also that $\mathrm{H}_{2}$ is very mobile on a grain surface, so that energy transfer is likely to occur between nearest neighbors. The mismatch in energy between donor and acceptor can be taken up by rotational energy of the $\mathrm{H}_{2}$ molecule phonons or center-of-mass translational motion. For the lower levels $\left(v-4000 \mathrm{~cm}^{-1}\right)$ this decay channel is probably closed because of the large energy mismatch.

Rotations might also directly act as energy acceptors of the excess vibrational energy. The experimentally measured relaxation rate of dipolar hydrides, which couple well with the lattice, ranges from about $10^{7} \mathrm{sec}^{-1}$ for the lighter ones $\left(e .8 .\right.$, OH and NH) to $10^{3} \mathrm{sec}^{-1}$ for the heavier ones (e.g., HCl; Legay 1977). The mass ratio of unity favors a high relaxation rate for this channel. However, because $\mathrm{H}_{2}$ is nonpolar,. it will not couple as well to the lattice. The transfer rate of vibration to rotation might perhaps be as fast as $10^{8} \mathrm{sec}^{-1}$. Small energy mismatches (i.e., less than one rotational spacing) can be taken up by center-of-mass translational motion of the $\mathrm{H}_{2}$ molecule or other local phonon modes. Decay of the rotational energy through transfer to local or delocalized phonon modes (one quantum at a time) will be very rapid because of the similarity in energy (for example, the Debye frequency of $\mathrm{H}_{2} \mathrm{O}$ is $133 \mathrm{~cm}^{-1}$ ). Some of the energy may wind up momentarily in vibrational energy of the $\mathrm{H}_{2}$-surface bond but, again, transfer to phonons will be rapid and buildup of energy in this mode will not occur. Thus, ejection of $\mathrm{H}_{2}$ is unlikely in this case.

Finally, direct transfer of the vibrational energy to center-ofmass translational motion has been estimated theoretically to have a rate of about $10^{7} \mathrm{sec}^{-1}$, but this estimate is very uncertain (Lucas and Ewing 1981). In this case, the excess energy in this channel is sufficient to cause ejection of a translationally "hot" ( $\approx 0.5 \mathrm{eV} ; 1 . e$. , one vibrational quantum) $\mathrm{H}_{2}$ molecule.

From this discussion, it seems that for the high vibrational levels of $\mathrm{H}_{2}$, decay will occur through energy transfer to neighboring molecules. The lower levels may decay through transfer to rotational modes or to center-of-mass translational motion. If the latter process dominates, then ejection is likely. Clearly, laboratory studies of the relaxation of vibrationally excited $\mathrm{H}_{2}$ are important to the resolution of this point. For heavier molecules, in particular for non-homonuclear molecules, energy transfer through multi-polar interaction is expected to dominate the reaction heat dissipation. 


\section{FORMATION OF $\mathrm{H}_{2}$ ON GRAIN SURFACES}

Ultraviolet observations have revealed that molecular hydrogen is an important constituent of diffuse interstellar clouds, such as those in front of $\zeta$ Oph (Morton 1975). It is generally assumed that $\mathrm{H}_{2}$ is the most abundant molecule in dense molecular clouds, but direct detection of $\mathrm{H}_{2}$ in quiescent clouds is difficult, because of the high UV opacity of such clouds and the low Einstein $A$ values of the IR rotational transitions. Ultraviolet-pumped, IR fluorescence from $\mathrm{H}_{2}$ has, however, been observed in reflection nebulae (e.8., NGC 2023) and around HII regions (e.g., the Orion bar; Gatley et al. 1986; Sellgren 1986). It has also been observed in regions with strong molecular shocks such as the $\mathrm{BN} / \mathrm{KL}$ region in Orion (Beckwith et al. 1978). This suggests that $\mathrm{H}_{2}$ is indeed the dominant form of hydrogen in molecular clouds.

It is difficult to form $\mathrm{H}_{2}$ through reactions in the gas phase and, therefore, it is generally presumed that it is formed on the surfaces of interstellar grains. Because of the importance of $\mathrm{H}_{2}$ in models of interstellar chemistry, the principles of grain-surface chemistry outlined above will be applied in this section to the formation of $\mathrm{H}_{2}$ in the interstellar medium. First, $\mathrm{H}_{2}$ formation on bare silicate grains in a diffuse interstellar cloud $\left(n_{0}-10 \mathrm{~cm}^{-3}, T-100 \mathrm{~K}\right.$, and $T_{d}-20 \mathrm{~K}$ ) will be considered, then $\mathrm{H}_{2}$ formation on the basal plane of graphite and amorphous carbon will be briefly examined. This will be followed by a discussion of poisoning of sites at which $H$ atoms can be chemisorbed. The section will close with a discussion of $\mathrm{H}_{2}$ formation on icy grain mantles inside molecular clouds. Hydrogen formation on organic refractory dust is presumably very similar to this latter case.

\section{$5.1 \mathrm{H}_{2}$ Formation on Silicate Surfaces}

Hydrogen atoms on a silicate grain can be chemisorbed as well as physically adsorbed on top of a chemisorbed layer (cf. \$4.2). There are about $10^{6}$ of each of these sites on a grain with a radius of $10^{3} \AA$. Under the conditions in the interstellar medium, migrating physically adsorbed atoms will react with chemisorbed atoms forming $\mathrm{H}_{2}$, which will evaporate quickly. With a sticking coefficient of unity and the gas parameters stated above, the $H$ accretion time-scale, $\tau_{a}$, on a $1000 \AA$ grain is about $500 \mathrm{sec}$. Thermal evaporation from chemisorption sites is completely unimportant. For physical adsorption sites the evaporation time-scale is about $2 \times 10^{4} \mathrm{sec}$. The migration time-scale for physically adsorbed atoms is, however, only $3 \times 10^{-9} \mathrm{sec}$ and, if there is an empty chemisorption site, the physically adsorbed atom will find it before it evaporates. Most $\mathrm{H}$ atoms will, thus, be bound in chemisorption sites. Occasionally, a chemisorbed atom can tunnel to an adjacent site on a time-scale ${ }^{i} t$ of about $10^{7} \mathrm{sec}$, assuming a barrier with a 
height of $1 \mathrm{eV}$ and a width of $1 \AA$. If this site is empty, the $H$ atom will be chemisorbed again until it tunnels on. If there is another $H$ atom present inthis site, the entering $H$ atom will only be physicall:" adsorbed. It may react with the $H$ atom already present or migrate on. With an activation barrier of $1000 \mathrm{~K}$ and a width of $1 \AA$, the time-scale for reaction, $\tau_{r}$, is $1.3 \times 10^{-6} \mathrm{sec}$. This should be compared to the time-scale for migration $\left(3 \times 10^{-9} \mathrm{sec}\right)$. The probability of reaction, $p_{r}$, is therefore only $2 \times 10^{-3}$ and the $H$ atom will migrate about 500 sites before it reacts (if it is not trapped in an empty chemisorbed site before that). The probability for evaporation during this migration and reaction time is given by $\tau_{r} / \tau_{e v}$; for the parameters above, this is negligible, and all accreting $\mathrm{H}$ atoms will form $\mathrm{H}_{2}$.

When the dust temperature is increased, evaporation of $H$ atoms in the physically adsorbed layer will become important. A critical temperature, $\mathrm{T}_{\mathrm{cr}}$, can be defined for which the probability for reaction with a chemisorbed atom is equal to the evaporation rate. This critical temperature is given by

$$
T_{c r}=E_{b} / \ln \left(\tau_{r} v_{z}\right)
$$

where $E_{b}$ is the binding energy and $v_{z}$ the characteristic vibration frequency of the $H$ atom in the physically adsorbed layer ( $c f$.

eq. [6]). For the values given above, this critical temperature is $50 \mathrm{~K}$. Note that this critical temperature is somewhat lower than that calculated by Hollenbach and Salpeter (1971). They envisioned a situation with a (small) number of enhanced binding sites interspersed in an otherwise physically adsorbed layer. However, they neglected the activation barrier for reaction. In the analysis above, the activation barrier for reaction is crucial. If the activation barrier for reaction were actually less than the binding energy of the $H$ atom in the physically absorbed site, then $\mathrm{H}_{2}$ formation would occur until evaporation from the chemisorbed layer dominated the loss of $\mathrm{H}$ atoms. The laboratory studies measure, however, both the physical adsorption energy and the activation energy rather directly, and the latter is indeed larger than the former. Above the critical temperature, the reaction rate will drop by a factor ${ }^{\tau} \mathrm{ev}^{\prime \tau_{\mathrm{r}}} \approx 3 \times 10^{-7} \mathrm{exp}\left(750 / \mathrm{T}_{\mathrm{q}}\right)$; at $75 \mathrm{~K}$, this factor is $6 \times 10^{-3}$. Finally when the dust temperature is very high and evaporation of physically adsorbed $H$ atoms is very rapid, only those impinging gas-phase atoms that have enough kinetic energy to directly overcome the activation barrier for reaction with the chemisorbed atoms will react. The rate of $\mathrm{H}_{2}$ formation is then geduced by a factor

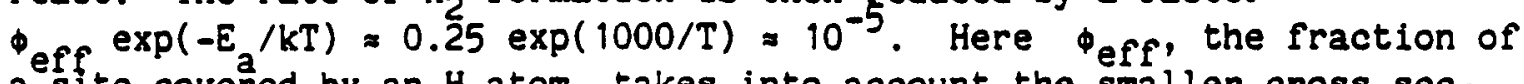
a site covered by an $\mathrm{H}$ atom, takes into account the smaller cross section of an $\mathrm{H}$ atom compared to the site area. Note that evaporation of 
chemisorbed $\mathrm{H}$ atoms is unimportant until the grain temperature reaches about $800 \mathrm{~K}$.

\section{$5.2 \mathrm{H}_{2}$ Formation on Graphite Surfaces}

The formation of $\mathrm{H}_{2}$ on the basal plane of graphite in the diffuse interstellar medium is very similar to that on silicates. As on silicates, atomic hydrogen can be both chemisorbed and physically adsorbed on graphite with binding energies of about $2 \mathrm{eV}$ and $0.07 \mathrm{eV}$, respectively (see \$4.2). The most important parameter for $\mathrm{H}_{2}$ formation, the activation barrier for the reaction between physically adsorbed and chemisorbed $\mathrm{H}$, is also very similar for the two surfaces ( $\approx 1200 \mathrm{~K}$; $\mathrm{King}$ and Wise 1963; versus $1000 \mathrm{~K}$; Wood and Wise 1962). The only difference is the presence of so-called "active surface areas" on graphite (i.e., the edges of the basal plane). Hydrogen atoms can attack the carbon skeleton at those areas and form saturated hydrocarbons such as methane. There is a considerable spread in the activation barriers reported for this set of reactions (1700-5000 K; King and Wise 1963; Wood and Wise 1969; Rye 1977; Balooch and Olander 1975), which may be due to differences in the surface structure of the samples (Veprek and Haque 1975). In any case, the activation energy is larger than that for reaction of a migrating $H$ atom with a chemisorbed $H$ atom on the basal plane; as a result, diffusing $\mathrm{H}$ atoms generally form $\mathrm{H}_{2}$ on the basal plane instead of reacting on the edges. This is even more pronounced in the interstellar medium because $H$ coverage on the basal plane will be high. In this respect, it should be noted that in the one series of experiments in which the $\mathrm{H}$ atom coverage on the basal plane was high, $\mathrm{H}_{2}$ was the dominant product at low temperatures (King and Wise 1963). Draine (1979) has argued that a migrating $\mathrm{H}$ atom would abstract a chemisorbed $\mathrm{H}$ atom (part of a $\mathrm{CH}_{3}$ group) on these edge structures to form $\mathrm{H}_{2}$ rather than form $\mathrm{CH}_{4}$ at low temperatures. However, this $\mathrm{H}$ abstraction reaction is likely to have considerable activation energy ( $\approx 8000 \mathrm{~K}$; Balooch and Olander 1975), and from the experiments we infer that it does not play a role until the surface temperature is much higher $(\approx 800 \mathrm{~K})$. At that temperature, $\mathrm{CH}_{4}$ becomes thermodynamically unstable, and the equilibrium will shift toward hydrogen and (solid) carbon (Wood and Wise 1969).

\subsection{Poisoning of Chemisorbed Sites}

We will now consider poisoning of the chemisorbed sites by other species. That this is important can be seen by comparing the accretion rate with the thermal evaporation rate. For small grains $(<100 \AA)$ or warm grains $\left(T_{d}>22 \mathrm{~K}\right)$, physically adsorbed $H$ atoms evaporate before the next $\mathrm{H}$ atom accretes, and $\mathrm{H}_{2}$ formation through recombination of two physically adsorbed $H$ atoms is inefficient. In the interstellar medium, 
poisoning will occur when another atom blocks the access of $H$ to the chemisorbed site. The poisoner may be physically adsorbed as long as it is essentially immobile. Note that if there are still a few chemisorbed sites available on a grain that $\mathrm{H}_{2}$ formation will occur by the mechanism outlined above. Poisoning is, thus, only effective if almost all of the sites are covered by poisoning atoms. The time-scale for poisoning is then the accretion time-scale per site of poisoners, which is about $5 \times 10^{5}\left(10^{-4} / x\right) \cdot y r$, with $x$ the relative gas-phase abundance of the poisoning atoms. For oxygen, nitrogen, and carbon atoms this time-scale is very short compared to the lifetime of the cloud or the $\mathrm{H}_{2}$ photodissociation lifetime, and they might therefore be very effective poisoners; this is even more likely because they are also chemisorbed to these sites. However, $\mathrm{H}$ atoms will be able to tunnel through the activation barrier for reaction and form $\mathrm{H}_{2} \mathrm{O}, \mathrm{NH}_{3}$, and $\mathrm{CH}_{4}$, which is energetically favorable. However, in the diffuse interstellar medium, photodesorption is an effective way of cleaning a surface of these molecules, since their photodesorption time-scale in an unshielded region is only about 700 yr (see \$9.1).

Poisoning by other atoms might also be important in the diffuse medium. The observed total surface area of interstellar dust is about $3 \times 10^{-21} \mathrm{~cm}^{2}$ per $\mathrm{H}$ atom. This number might be somewhat larger (50\%) if the grain size distribution extends to below $100 \AA$. With a surface area per site of about $7 \AA^{2}$, this dust surface area corresponds to about $4 \times 10^{-6}$ sites per $H$ atom. Thus, species with a total gas-phase abundance in the diffuse interstellar medium comparable to this number could poison the chemisorbed sites on a grain surface. Note that we have to correct for the underlying depletion caused by the incorporation of some elements in refractory dust grains produced by stars. This implies that poisoning by atoms such as $\mathrm{S}, \mathrm{Si}, \mathrm{Na}, \mathrm{Mg}, \mathrm{Fe}, \mathrm{Al}, \mathrm{Ca}$, and $\mathrm{Ni}$ can be of importance. Some of these (e.g., S and $\mathrm{Si}$ ) will form volatile hydrides which are probably also easily photodesorbed, whereas others (i.e., Na and $\mathrm{K}$ ) may be ejected upon electron recombination (cf. \$6). The transition metals, as well as $\mathrm{Ca}$, will form hydroxide groups on interstellar grain surfaces $(\$ 6)$. Presumably, $H$ will also be chemisorbed on such surfaces. Moreover, although their abundance might be sufficient to completely poison the chemisorbed sites on a time-scale of only about $10^{7} \mathrm{yr}$, this assumes that these species are used as efficiently as possible (i.e., one atom per chemisorbed site). This is unlikely, since these species are immobile at $20 \mathrm{~K}$. It seems, therefore, that poisoning of grain surfaces cannot stop $\mathrm{H}_{2}$ formation in the diffuse interstellar medium. 


\section{$5.4 \mathrm{H}_{2}$ Formation on Grain Mantles}

Finally, $\mathrm{H}_{2}$ formation on icy surfaces will be considered. Inside dense molecular clouds, interstellar grains.will accrete mantles consisting of such simple molecules as $\mathrm{H}_{2} \mathrm{O}, \mathrm{NH}_{3}, \mathrm{CO}$, and $\mathrm{CH}_{3} \mathrm{OH}$ (see $\$ 7$ ). Such icy grain mantles have only physical adsorption sites, with a typical binding energy of about $350 \mathrm{~K}$; this is owing to the high surface coverage of $\mathrm{H}_{2}$ (Govers et al. 1980; Tielens and Hagen 1982; see also \$4.2). The thermal evaporation rate of $H$ is then about $2 \times 10^{2} / \mathrm{sec}$, which is lower than the accretion time-scale of $\mathrm{H}$ atoms on a grain $\left(-10^{5} \mathrm{sec}\right.$ for a $1000 \AA$ grain and a typical $\mathrm{H}$ density of $1 \mathrm{~cm}^{-3}$ ) and $\mathrm{H}_{2}$ formation by direct recombination of physically adsorbed $H$ atoms on a grain surface will not occur. Of course, by the time a grain mantle has been formed, hydrogen is already completely converted into the molecular form through reactions on bare graphite or silicate grain surfaces.

Although icy grain mantles do not have chemisorbed sites, molecules such as $\mathrm{H}_{2} \mathrm{CO}, \mathrm{H}_{2} \mathrm{~S}, \mathrm{~N}_{2} \mathrm{H}_{2}, \mathrm{~N}_{2} \mathrm{H}_{4}$, and metal hydrides can act as enhanced binding sites for $\mathrm{H}_{2}$ formation (Tielens and Hagen 1982). During its lifetime on a grain surface, an $\mathrm{H}$ atom may tunnel through the activation barrier associated with $\mathrm{H}$ abstraction from such molecules producing $\mathrm{H}_{2}$ and leaving a reaction site (cf. $\$ 4.5$ ). Thus, $\mathrm{H}_{2}$ can also be formed and reformed inside dense molecular clouds: It should be stressed that because radical-radical reactions are activationless, if there are radicals on the surface, then an $H$ atom will react with them rather than tunnel through an activation barrier and form $\mathrm{H}_{2}$. Reaction of $\mathrm{H}$ with non-radicals such as $\mathrm{O}_{2}$ and $\mathrm{CO}$ can also be important in this respect. Thus, when the gas-phase atomic $H$ abundance decreases below the abundance of heavier species such as $\mathrm{O}, \mathrm{CO}$, and $\mathrm{O}_{2}, \mathrm{H}_{2}$ formation on grain surfaces will stop ( $T$ ielens 1983b). Typically, this occurs at a density of about $3 \times 10^{4} \mathrm{~cm}^{-3}$ (inert conditions; see \$7). This will, however, not increase the abundance of $\mathrm{H}$ in the gas phase, because the $\mathrm{H}$ atom will now be incorporated in such molecules as $\mathrm{H}_{2} \mathrm{O}, \mathrm{NH}_{3}$, and $\mathrm{H}_{2} \mathrm{CO}$.

\section{DEPLETION OF THE ELEMENTS}

UV absorption line studies have shown that the gas-phase abundance of many elements in the diffuse interstellar medium is less than expected from cosmic abundances. The degree of depletion varies from element to element, as well as from one line of sight to another (see chapter by Jenkins, elsewhere in this volume). The missing elemental fraction is generally thought to be locked up in grains and, thus, elemental abundance studies may yield information on the composition and physical and chemical properties of interstellar grains. Several processes can influence the gas-phase abundance of an element. Clearly, dust 
condensation in the high-temperature and high-density outflow from stellar sources (Field 1974) is important, as evidenced, for example, by the large fraction of silicon locked up in silicates (cf. \$1.1). Destruction of dust in interstellar shocks is also important, given the relatively high gas-phase abundance of elements such as $\mathrm{Ca}, \mathrm{Fe}$, and $\mathrm{Si}$ in high-velocity clouds (i.e., recently shocked clouds) compared to the general diffuse interstellar medium (Spitzer 1976). Finally, accretion of atoms in the interstellar medium is also an important process (Snow 1975), since elemental abundances lower than $10^{-1}$ times the cosmic abundance are difficult to achieve by stardust formation alone (see chapter by Jura, elsewhere in this volume). The complexity of these interplaying processes has defied a general theory of elemental depletions in the interstellar medium. Considering the scope of this book, in this section we merely want to point out some considerations concerning the third process, the accretion of elements in the diffuse interstellar medium.

Nitrogen, oxygen, and sulfur are expected to be little depleted in the interstellar medium, because their main condensation products around stars are very volatile $\left(\mathrm{e} . \mathrm{g} ., \mathrm{NH}_{3}, \mathrm{H}_{2} \mathrm{O}\right.$, and $\left.\mathrm{H}_{2} \mathrm{~S}\right)$. Although these elements are presumably chemisorbed on interstellar graphite and silicate surfaces, they are expected to react in the diffuse medium with atomic hydrogen on grain surfaces and thereby form their volatile hydrides. These are probably easily photodesorbed (cf. \$9). Once they are in the gas phase, photodissociation will rapidly destroy these molecules. Sputtering in low velocity $(=20 \mathrm{~km} / \mathrm{sec})$ shocks can also be of importance for the removal of these saturated molecules. Some oxygen will be locked up in refractory silicate grains and, possibly, in organic refractory material.

Carbon takes a special place among the more abundant heavy elements. Not only is solid carbon the thermodynamically favored phase at high temperatures and densities (i.e., in the outflow froms stars), but carbon can also form large molecules that can be fairly resistant to destruction. Observations indicate that a considerable fraction of the carbon can be locked up in grains made up of cross-linked macromolecules (cf. 1.3). The physical processes important in the formation of the organic refractory dust component in the interstellar medium are discussed in $\$ 8$. Here we merely emphasize that carbon can be highly depleted in the interstellar medium.

Silicon and phosphorous can form a refractory high-temperature condensate in the outflow around stellar objects (i.e., $\mathrm{MgSiO}_{3}$ and $\mathrm{Fe}_{3} \mathrm{P}$; Fegley and Lewis 1980). In the diffuse interstellar medium, it is expected, however, that both atoms when accreted on a grain surface will form saturated, volatile hydrides (i.e., $\mathrm{SiH}_{4}$ and $\mathrm{PH}_{3}$ ) in a manner similar to that of carbon and nitrogen. Again, photodesorption will probably prevent large depletions in the diffuse medium. Silicon can 
substitute for carbon in organic molecules, and its depletion in the interstellar medium may, therefore, vary through incorporation into the organic refractory dust component formed in molecular clouds (cf. \$8).

The depletion pattern of the alkali and alkaline metals is somewhat puzzling in view of their closeness in the periodical system. Sodium, potassium, and magnesium are little (about a factor of two) depleted in stardust (presumably composed of silicates) and do not show large differential depletion effects in the diffuse interstellar medium. In contrast, $\mathrm{Ca}$ is highly depleted and shows large variations. Both $\mathrm{Na}$ and $K$ have ionization potentials below the grain work function and, thus, charge effects will not complicate their sticking (i.e., $S \approx 1$; cf. \$4.3). The positive ions $\mathrm{Na}^{+}$and $\mathrm{K}^{+}$have an inert-gas electronic structure, which inhibits their reaction with $H$ upon accretion. However, once accreted an electron is expected to be able to tunnel through the work function and recombine with the alkali metal ion. If this recombination occurs in a Rydberg state (high principal quantum number), the extended electron cloud coupled with the repulsive interaction with the surface will then, presumably, lead to desorption (see the discussion on photodesorption in \$9.1). Recombination may also occur directly in an antibonding valence orbital of the surface-adion bond. If this scenario is correct, then one would expect that because of their similar electronic structures and low ionization potentials, Li, Rb, and Cs are also little depleted in the interstellar medium.

Because the ionization potentials of $\mathrm{Mg}_{\mathrm{g}}$ and $\mathrm{Ca}$ lie above the work function of interstellar grains, sticking wight be complicated by recombination effects (cf. \$4.3). The large depletion of Ca suggests, however, that electrons in interstellar grains are not very mobile on the short collision time-scale $\left(=10^{-13} \mathrm{sec}\right)$. Since the cations do not have an inert-gas electron structure, they probably react immediately upon accretion with atomic hydrogen on the surface, which may actually prevent their ejection upon electron recombination. Both elements will react with hydrogen to form the dihydrides. However, whereas $\mathrm{MgH}_{2}$ has a covalent structure, $\mathrm{CaH}_{2}$ is a salt (Mueller, Blackledge, and Libowitz 1968). Presumably, $\mathrm{MgH}_{2}$ is therefore easily photodesorbed and $\mathrm{CaH}_{2}$ is not. If this is indeed the essential difference, then it is expected that other elements that form covalent hydrides, such as $B e, 2 n$, and $C d$, would be little depleted ( $\mathrm{Zn}$ is discussed below). At this point, it should be recalled that hydrogen abstraction reactions are very important on interstellar grain surfaces. The $\mathrm{Ca}$ hydride remaining on the surface will therefore react often with $\mathrm{H}$ (i.e., $\mathrm{CaH}_{2}+\mathrm{H} \rightarrow \mathrm{CaH}+\mathrm{H}_{2}$, followed by $\left.\mathrm{CaH}+\mathrm{H} \rightarrow \mathrm{CaH}_{2}\right)$. This cycle will continue until $\mathrm{CaH}$ is poisoned by another atom, presumably 0 , leading to hydroxide formation. It is expected that the $\mathrm{Ca}(\mathrm{OH})_{2}$ will be bonded quite strongly to the surface. 
The transition metals $\mathrm{Ti}, \mathrm{Mn}, \mathrm{Fe}$, and $\mathrm{Ni}$ are highly depleted in the interstellar medium. In contrast, the depletion of $2 n$ is quite small. The special feature of the electronic structure of the transition metals that presumably lead to this depletion pattern is the presence of unfilled $d$ orbitals, which can form strong complexes with electron-rich material (Pauling 1964). The unfilled 3d orbitals available should provide strong bonds to a grain surface. These elements can also react with atomic $H$ on the surface to form mono- and dihydrides, which could be stabilized by the formation of a coordination complex with a ligand group on the surface (e.g., highly polar or polarizable groups with unshared electron pairs such as an $\mathrm{OH}$ group in a silanol moiety on a silicate surface or the $\pi$ electron system in PAHs, amorphous carbon particles, or graphite). This complex will still be strongly bound to a grain surface and photodesorption will be of little importance. In this respect it is important to note that $2 \mathrm{n}$ has all its $3 d$ orbitals filled and does not readily form coordination complexes. Zinc hydride has a covalent character (Mueller, Blackledge, and Libowitz 1968), and is probably easily photodesorbed or sputtered. Cadmium, which has an electronic structure similar to that of $\mathrm{Zn}$, also forms a covalent hydride and should, therefore, have a similar depletion pattern.

In summary, the differential elemental depletion patterns in the diffuse interstellar medium can be ascribed to the chemical properties of the elements. Of particular importance is the ability to form hydrides and coordination complexes. Photodesorption and electron recombination may play a key role in preventing depletion of some elements. In contrast to Barlow (1978), we do not think that ejection upon reaction is an important process ( $c$. \$4.6). We also caution against the straightforward use of surface bond energies of the elements based on chemisorption theory to explain observed depletion patterns. Chemisorption is a very complex process and is far from theoretically understood (Einstein et al. 1980).

\section{COMPOSITION OF ICY GRAIN MANTLES}

The composition of interstellar grain mantles has been calculated, employing gas-phase as well as grain-surface reactions (Tielens and Hagen 1982; Tielens 1983b; d'Hendecourt, Allamandola, and Greenberg 1985). Figure 13 shows the composition of the gas phase and the grain mantle as a function of the gas-phase hydrogen density (Tielens and Hagen 1982). In the subsequent discussion we will concentrate on the global composition of the grain mantles and ignore to a large extent the abundances of trace species.

First, we will concentrate on the gas-phase composition of molecular clouds (Fig. 13a), since it sets the stage for the diffusion-limited 

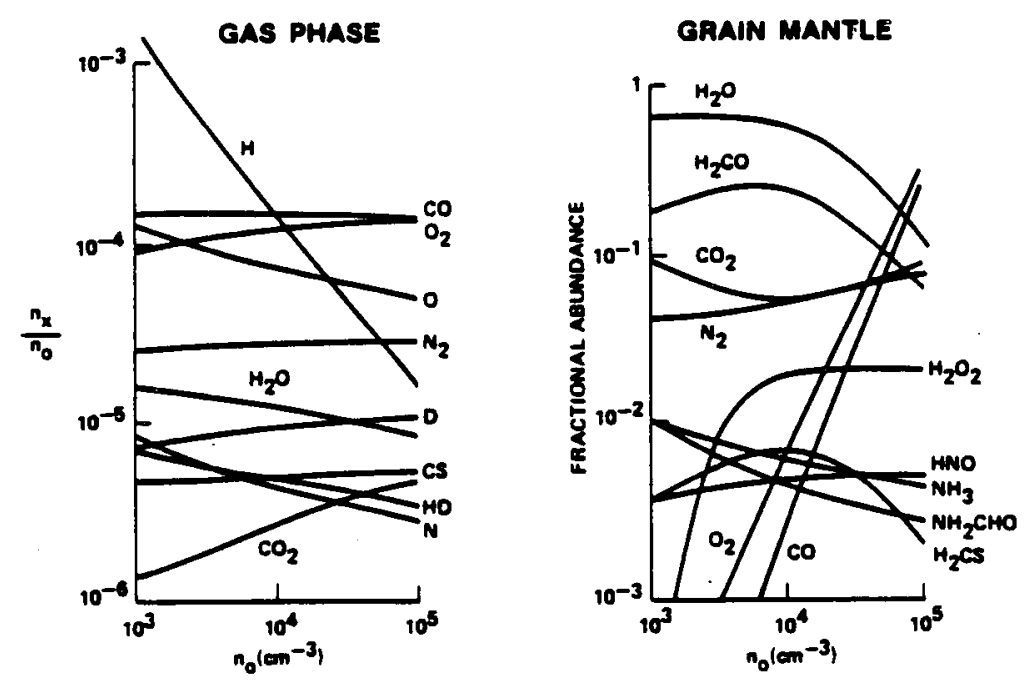

Eigure 13. Molecular composition of interstellar clouds as a function of density in the gas phase (a) and grain mantle (b).

chemistry on grain surfaces. Hydrogen is mainly in molecular form $\left(\mathrm{H}_{2}\right)$. The atomic hydrogen abundance is inversely proportional to the total density, because the $\mathrm{H}_{2}$ formation time-scale depends on the (grain) density, whereas the destruction time-scale (by cosmic rays) does not. The most abundant molecule in the gas phase after $\mathrm{H}_{2}$ is $\mathrm{CO}$, which contains most of the elemental carbon in the gas phase. Oxygen and nitrogen are either in atomic or molecular form $\left(\mathrm{O}_{2}\right.$ and $\left.\mathrm{N}_{2}\right)$. Note that $\mathrm{H}_{2} \mathrm{O}$ contains only a small fraction of the gas-phase oxygen. These results are very typical for time-independent gas-phase chemistry calculations or for time-dependent gas-phase chemistry calculations at late stages ( $>10^{6}$ yr; Mitchell et al. 1978; Prasad and Huntress 1980; Graedel et al. 1982).

The calculated grain mantles consist mainly of the molecules $\mathrm{H}_{2} \mathrm{O}$, $\mathrm{H}_{2} \mathrm{CO}, \mathrm{N}_{2}, \mathrm{O}_{2}, \mathrm{CO}, \mathrm{CO}_{2}, \mathrm{H}_{2} \mathrm{O}_{2}$, and $\mathrm{NH}_{3}$ and their deuterated counterparts in varying ratios. The exact composition depends strongly on the conditions in the gas phase. Depending on the gas-phase composition, three different physical regimes can be discerned, a reducing, an inert, and an oxiding "atmosphere." For low densities ( $n<5 \times 10^{4} \mathrm{~cm}^{-3}$ ), atomic hydrogen is more abundant in the gas phase than the heavier species (e.g., $0, \mathrm{O}_{2}$, and $\mathrm{CO}$ ) and the gas is largely reducing. As a result, most species will be hydrogenated on the grain surface (e.g., $\mathrm{H}_{2} \mathrm{O}$ and $\mathrm{H}_{2} \mathrm{CO}$ ). Unsaturated molecules, such as $\mathrm{CO}$, are trace species in the grain mantle, whose abundance depends directly on their gas-phase abundance relative to that of atomic $H$ and is, thus, a strong function of the total hydrogen density. An exception should be made for $\mathrm{N}_{2}$, which 
does not react with atomic $H$. Ammonia is therefore a trace species, whose abundance depends on the gas-phase abundance of atomic $N$.

At higher densities $\left(\mathrm{n}>5 \times 10^{4} \mathrm{~cm}^{-3}\right)$, the atomic $\mathrm{H}$ density in the gas phase is less than that of the heavier species and most accreting species will not be hydrogenated. The resulting grain mantle depends then on the 0 to $\mathrm{O}_{2}$ ratio in the gas phase, since 0 is the next most abundant, mobile radical. If this ratio is low, then only few grain surface reactions will occur (i.e., inert atmosphere). The grain mantle will then mainly reflect the gas-phase composition (e.g., $\mathrm{CO}, \mathrm{O}_{2}$, and $\mathrm{N}_{2}$ ). If the atomic 0 to $\mathrm{O}_{2}$ ratio in the gas phase is high (oxidizing atmosphere) then atomic 0 and $\mathrm{CO}$ will react; $\mathrm{CO}_{2}$ will then be the dominant grain-mantle constituent. In both cases hydrogenated molecules such as $\mathrm{H}_{2} \mathrm{O}$ and $\mathrm{H}_{2} \mathrm{CO}$ are only trace species, whose abundance depends on their abundance in the gas phase and on the ratio of atomic $H$ to heavier species.

Infrared observations show that interstellar grain mantles consist mainly of $\mathrm{H}_{2} \mathrm{O}$ and $\mathrm{CH}_{3} \mathrm{OH}$; $\mathrm{CO}$ and $\mathrm{NH}_{3}$ are only trace species (Tielens et al. 1986a). This implies that grain mantles are formed in a "reducing" atmosphere and, thus, at relatively low densities $\left(\approx 10^{4} \mathrm{~cm}^{-3}\right)$. Note that $\mathrm{CH}_{3} \mathrm{OH}$ may result from $\mathrm{H}$ addition reactions to $\mathrm{H}_{2} \mathrm{CO}$. There is some experimental justification for the occurrence of this reaction at low temperatures ( $T$ ielens and Hagen 1982). It has, however, not been included in the calculations described above. Finally, in some cases evidence for grain mantle formation in a more inert atmosphere has also been observed ( $T$ ielens et al. 1986á).

It is interesting to consider the deuteration of species in a grain mantle. Figure 14 shows the relative abundance of a deuterated species with respect to its hydrogenated counterpart (Tlelens 1983b). Generally, this ratio reflects the ratio of atomic deuterium to atomic hydrogen in the gas phase, essentially because the grain-surface reaction networks are very similar. Thus, this ratio increases strongly with increasing gas-phase density owing to the decrease in the gas-phase abundance of atomic hydrogen ( $f$. Fig. 13a). It is important to note that although most of the hydrogen is in molecular form, most of the deuterium is actually in atomic form in the gas phase, and the ratio of atomic deuterium to atomic hydrogen is much larger than expected from elemental abundances ( $T$ ielens 1983b). Molecular hydrogen is destroyed in the gas phase by cosmic ray ionization leading eventually to $\mathrm{H}_{3}^{+}$. However, in its destruction network, HD competes with other molecules such as $\mathrm{CO}$ and $\mathrm{N}_{2}$ for reaction with $\mathrm{H}_{3}^{+}$forming $\mathrm{H}_{2} \mathrm{D}^{+}$. Reaction of this molecular ion with neutral molecules such as $\mathrm{CO}$ and $\mathrm{N}_{2}$ (leading to the deuterated ions $\mathrm{DCO}^{+}$and $\mathrm{N}_{2} \mathrm{D}^{+}$), followed by dissociative electron recombination produces atomic deuterium. On the basis of their relative abundances, one out of about every 20 cosmic ray ionizations of $\mathrm{H}_{2}$ will lead to HD destruction; thus, the HD destruction rate is comparatively 


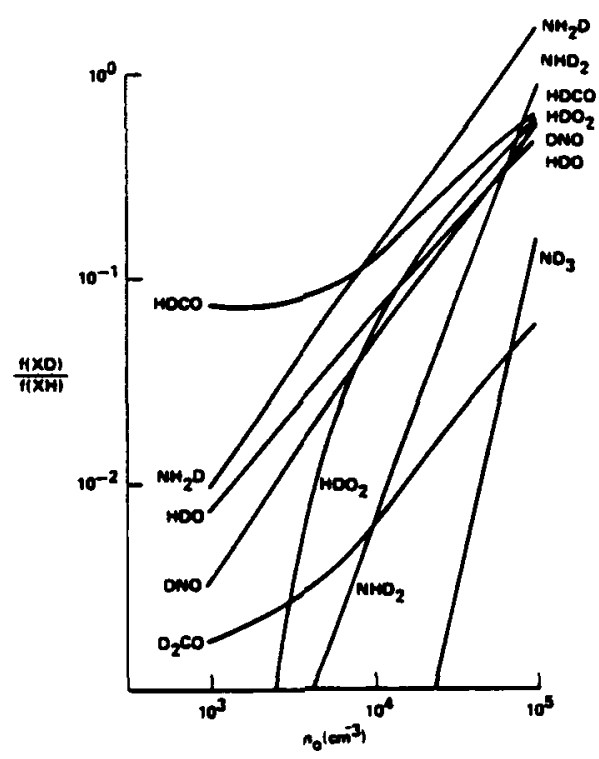

Figure 14. Concentration of the most abundant deuterated molecules relative to their hydrogenated counterparts in the grain mantle as a function of the total hydrogen density in the gas phase.

much larger than that of $\mathrm{H}_{2}$ (Tielens 1983ㅁ). In fact the high deuteration observed for molecular ions in molecular clouds implies that the $D / H$ ratio is much larger than the elemental abundance ratio. Note that the grain-mantle abundances of some molecules $\left(e .8 ., \mathrm{HDCO}, \mathrm{D}_{2} \mathrm{CO}\right.$ and to a lesser extent HDO) do not directly reflect the atomic $D / H$ ratio in the gas phase. These result from differences in the grain-surface reaction network for these species (e.g., H abstraction reactions are of importance; Tielens 1983b).

8. UV PHOTOLYSIS, TRANSIENT HEATING, AND ORGANIC REERACTORY DUST

Greenberg and his co-workers and others (Greenberg 1963; Greenberg and Yencha 1973; Hagen et al. 1979; Greenberg 1979; d'Hendecourt et al. 1986; Donn 1960; Donn and Jackson 1970; Khare and Sagan 1973) have suggested that UV photolysis of interstellar grain mantles inside molecular clouds can produce complex molecules. These complex molecules are not as easily destroyed by interstellar shock waves as $\mathrm{H}_{2} \mathrm{O}$ ice (Draine and Salpeter 1979) and could conceivably form an important component of the dust in the diffuse interstellar medium. Processing by cosmic rays might play a similar role (Moore and Donn 1982; Strazulla et al. 1983). Infrared spectroscopy of protostars deeply embedded inside dense molecular clouds suggests that the composition of interstellar grain mantles is more complex than can be explained by grain-surface reactions 
alone (Lacy et al. 1984; Tielens et al. 1984, 1986a). This may indicate the importance of energetic processing by UV photons or cosmic rays.

It should be stressed that grain-surface reactions alone only for.a simple, saturated hydrides (cf. \$7). The formation of the organic refractory dust component, consisting of long carbon chains (i.e., polymeric carbon), in such a reducing atmosphere as the interstellar medium is difficult to understand. This section will discuss this problem and will identify the two key processes--that is, UV photolysis producing radicals and radical diffusion triggered by transient heating--that may convert interstellar grain mantles consisting of simple saturated hydrides into the complex organic refractory dust component of the interstellar medium.

In contrast to grain-surface reactions occurring during accretion of gas-phase species, UV photolysis mainly takes place in the bulk. Ultraviolet photolysis of interstellar grain mantles can produce reactive radicals, often in electronically or vibrationally excited states (Hagen 1982). These "hot" radicals can react with their neighbors or they can diffuse through the matrix and react with other species present (Hagen, Allamandola, and Greenberg 1979). Because of the large amount of excess energy available, even reactions with molecules having closed electron shells, which normally have appreciable activation barriers; might take place. Because, in general, reactions only take place when the reactants approach along well-defined reaction paths and because collisions of the hot reactant and the host material will transfer energy to the matrix (e.g., heat), reactive species can also be stored at low temperatures in the grain mantle. Upon warm-up those radicals can start diffusing through the host material and react with each other. Thus, during photolysis and warm-up complex molecules can build up, producing an organic refractory mantle on interstellar dust grains (Greenberg 1979).

The chemical processes taking place during grain mantle photolysis are complex. Often there are several photo-decomposition channels of a primary molecule. The photoproducts can also react with many of the neighboring unsaturated molecules. Moreover, the resulting secondary molecules themselves can often also be photolyzed, leading to a third generation of molecules. This complexity is compounded by the loss of volatile components owing to temperature spikes generated by grain-grain collisions (d'Hendecourt et al. 1982) or cosmic rays (Leger et al. 1985). The complexity of these processes has inhibited theoretical studies of photolyzed grain mantles. Extensive and painstaking laboratory studies are required to understand these processes. Although some laboratory studies have already been undertaken (Hagen 1982; d'Hendecourt et al. 1986; van de Bult 1986), this field is still in its infancy. Little more beyond the general outline of the chemical scheme (UV photons produce radicals which form complex molecules) can presently 
be said. Among the typical radicals produced by the photolysis of $\mathrm{H}_{2} \mathrm{O}$ rich ices are $\mathrm{HCO}, \mathrm{HOCO}$, and $\mathrm{OH}$. Simple molecules that have been produced by photolysis of such mixtures are $\mathrm{H}_{2} \mathrm{CO}$ and $\mathrm{CO}_{2}$ (Hagen 1982; d'Hendecourt et al. 1986). A general conclusion seems to be that aliphatic rather than aromatic hydrocarbon mixtures are readily formed. Ultraviolet photolysis of oxygen-rich mixtures generally leads to the formation of residues containing large amounts of carboxylic acids. If $\mathrm{NH}_{3}$ is also present in the initial mixture, then amino groups are also formed (Hagen, Allamandola, and Greenberg 1979; d'Hendecourt et al. 1986).

Let us consider quantitatively the conversion of interstellar grain mantles into organic refractory dust by means of UV photolysis. The absorption cross section of $\mathrm{H}_{2} \mathrm{O}$ ice below $1600 \AA$ is about $10^{3} \&$ mole $e^{-1} \mathrm{~cm}^{-1}$, about 4 times larger than for gas-phase $\mathrm{H}_{2} \mathrm{O}$. In an unattenuated interstellar FUV radiation field $\left(10^{8}\right.$ photons $\mathrm{cm}^{-2} \mathrm{sec}^{-1}$; Habing 1968), the number of photons absorbed by one $\mathrm{H}_{2} \mathrm{O}$ molecule in a grain mantle, $\mathrm{N}_{\text {abs }}\left(A_{v}=0\right)$, is then about $2 \times 10^{-9} \mathrm{sec}^{-1}$. Taking a UV dust albedo of 0.6 , a mean cosine of the scattering angle of the dust of 0.7 (Lillie and Witt 1976), and a UV dust absorption cross section 3 times the visual extinction, $A_{v}$, the number of UV photons absorbed by one $\mathrm{H}_{2} \mathrm{O}$ molecule in a grain mantle as a function of depth in a semiinfinite cloud is given by (Elannery et al. 1980) as

$$
N_{a b s}\left(A_{v}\right)=2 \times 10^{-9} \exp \left(-2 A_{v}\right) \text {. }
$$

Thus, deep inside dense clouds $\left(A_{v}>7 \mathrm{mag}\right)$ UV photolysis by the ambient interstellar radiation field is unimportant over the lifetime of a molecular cloud $\left(3 \times 10^{7} \mathrm{yr}\right)$. The UV photon field inside a dense cloud may actually be larger than estimated from this treatment because of the presence of local UV sources. For example, cosmic ray excitation of $\mathrm{H}_{2}$ may lead to a mean intensity of the UV radiation field inside the cloud of $10^{3}$ photons $\mathrm{cm}^{-2} \mathrm{sec}^{-1}$ (Prasad and Tarafdar 1983). Thus, for a visual extinction larger than about 6 mag this field will dominate. There may also be other sources of UV photons inside dense clouds, such as shocks or newly formed stars, whose flux is difficult to estimate but which might be as high as $5 \times 10^{3}$ photons $\mathrm{cm}^{-2} \mathrm{sec}^{-1}$ (Norman and Silk 1980). Taking $10^{3}$ photons $\mathrm{cm}^{-2} \mathrm{sec}^{-1}$ as the value for the mean intensity of the UV radiation field inside dense clouds yields, for the number of UV photons absorbed by one $\mathrm{H}_{2} \mathrm{O}$ molecule in a grain mantle, $N_{\text {abs }}=2 \times 10^{-14} \mathrm{sec}^{-1}$. Thus, over the lifetime of a molecular cloud every $\mathrm{H}_{2} \mathrm{O}$ molecule in a grain mantle will have absorbed about $20 \mathrm{UV}$ photons.

Not all of the absorbed UV photons will, however, lead to radical production. The efficiency, $f_{u v}$, of the photodissociation reaction (number of radicals produced per UV photon absorbed) depends on the 
particular electronic state involved. In the gas phase, photodissociation is the dominant channel (Okabe 1978; Calvert and Pitts 1966); in a solid, many other decay channels compete. These include photo-assisted reactions with neighbors, and dissipation of the energy in the phonon modes of the solid (i.e., heat; Bass and Broida 1966; Bondybey and Brus 1980). The accessibility of the various channels depends on the composition of the ice, the identity of the molecule that has absorbed the UV photon, and the particular state it is excited into. Considering $\mathrm{H}_{2} \mathrm{O}$ under a few well-defined conditions, several pathways can be identif and followed. The dissociated $\mathrm{H}_{2} \mathrm{O}$ molecule may recombine immediately; the "hot" product may recombine with a neighbor to form a new species and it may also recombine with another radical to reform the original molecule. In that case, the UV photon has merely promoted diffusion of a previously stored radical. The importance of this latter process depends on the average distance between stored radicals as compared to the average distance a hot product will diffuse before it is trapped. Note that this process will limit the concentration of radicals that can be stored in a grain mantle. Laboratory studies of UV photolysis of $\mathrm{H}_{2} \mathrm{O}$ and $\mathrm{CO}$ matrices yield values for $f_{y v}$ ranging from 0.25 to 0.005 when the $\mathrm{H}_{2} \mathrm{O} / \mathrm{CO}$ ratio increases from $10^{-2}$ to 2 (Hagen 1982). In the former case, the $\mathrm{H}$ and $\mathrm{OH}$ produced will react with a $\mathrm{CO}$, producing $\mathrm{HCO}$ and $\mathrm{HOCO}$. In the latter case, there is a much greater probability of reaction of the $\mathrm{H}$ with an $\mathrm{OH}$ previously produced, thereby reforming $\mathrm{H}_{2} \mathrm{O}$. The cage effect might limit $f_{\text {uv }}$, thus, to values less than 0.25 . For the lower $C O$ concentrations, the efficiency is limited by the recombination of the hot product reforming $\mathrm{H}_{2} \mathrm{O}$.

The limiting concentration of $\mathrm{H}_{2} \mathrm{O}$ photodissociation products in a pure $\mathrm{H}_{2} \mathrm{O}$ matrix is estimated to be about 19 from these experiments (Hagen 1982). In photolysis experiments involving more complex mixtures designed to duplicate an interstellar grain mantle, the conversion efficiency also falls in the $1 \%$ range (d'Handecourt et al. 1986). Water is a major component of interstellar grain mantles (Tielens and Hagen 1982; Tielens et al. 1986a). On the basis of these experiments, the UV photolysis efficiency for interstellar grain mantles is about $0.5 \%$. With this efficiency, about $10 \%$ of the mantle molecules will have been converted into photolyzed products over the lifetime of the cloud. Thus, while photolysis will not change the composition of accreted grain mantles drastically it can increase the complexity of the molecules in a grain mantle. It should be emphasized, however, that most of these photolyzed products will not be very large. In particular, photolysis of $\mathrm{H}_{2} \mathrm{O}$ will lead to reactions of its photolysis products, $\mathrm{H}, \mathrm{O}$, and $\mathrm{OH}$ with neighboring simple molecules such as $\mathrm{CO}$, thereby forming $\mathrm{CO}_{2}, \mathrm{H}_{2} \mathrm{CO}$, and $\mathrm{CH}_{3} \mathrm{OH}$. In an interstellar grain mantle, molecules containing $\mathrm{CH}_{2}$, $\mathrm{CH}_{3}$, and $\mathrm{NH}_{2}$ groups can also be photolyzed, adding to the complexity of the mixture. However, most product molecules are expected to be 
volatile and will not survive long in the harsh environment of the diffuse interstellar medium. The efficiency of the formation of very complex molecules is expected to be low. Using the values quoted above, for a molecule containing $N$ building blocks, $f_{u v} \approx 20(0.005)^{n}$, where $n={ }^{2} \log N$. UV photolysis alone can, therefore, not account for the presence of an abundant complex organic refractory dust component in the interstellar medium.

Laboratory studies show that after warm-up of a photolyzed icy mixture, a complex molecular mixture remains. Presumably, this mixture has been partly formed by radical diffusion and reaction during the warm-up (the exploding grain mantle; see \$9.1). However, the efficiency of this process has not been estimated. For the sake of argument we will assume that $1 \%$ of all the radicals present at the warm-up will form such complex molecules (i.e., $10^{-4}$ of all the molecules in the grain mantle). The equilibrium concentration of radicals in an $\mathrm{H}_{2} \mathrm{O}-\mathrm{rich}$ matrix $(\approx 1 \%)$ is attained on a time-scale of about $10^{5} \mathrm{yr}$ inside a dense cloud, which is fast compared to the accretion time-scale $\left(10^{9} / \mathrm{n}_{0} \mathrm{yr}\right)$. Warm-up of the grain mantles may be due to grain-grain collisions (Greenberg 1979) with a time-scale of about $10^{8} /\left(\mathrm{n}_{0}\right)^{1 / 2} \mathrm{yr}$ in molecular clouds (Draine 1984). It has also been suggested that warm-up results from transient heating by cosmic rays with a time-scale of about $3 \times 10^{4}$ yr (see \$9.1). The limiting step seems, thus, to be the accretion on grains. Taking an accretion time-scale of $10^{6} \mathrm{yr}$, appropriate for a density of $10^{3} \mathrm{~cm}^{-3}$ and a cloud lifetime of $3 \times 10^{7} \mathrm{yr}$, implies that each atom cycles about 30 times through a grain mantle and thus has 0.37 probability of being incorporated into the organic refractory dust. Since an atom cycles about 50 times through a molecular cloud during its lifetime in the interstellar medium, there is a $15 \%$ probability that it will be part of the organic refractory dust component during some part of its lifetime.

It should be emphasized that there are two key steps in models of the UV photolysis of icy grain mantles and the production of complex organic refractory material. First, such models hinge on the presence of sources of UV radiation inside a dense cloud. Given a cloud lifetime of $3 \times 10^{7} \mathrm{yr}$, the ambient interstellar UV radiation field is unimportant for UV photolysis when $A_{y}$ is larger than about 7 mag. Second, transient heating of grain mantles is required to bring the stored radicals together for reaction. The efficiency of such explosions is, however, unknown. A conversion efficiency of about $1 \%$ of the stored radicals is required to explain the presence of about $25 \%$ of the carbon in the form of organic refractory dust. 


\section{GRAINS AND GAS-PHASE COMPOSITION OE MOLECULAR CLOUDS}

One of the most pressing problems remaining in theoretical studies of the gas-phase composition of molecular clouds is the contribution of the grain chemistry. Grains can act as sources and as sinks for gas-phase species. The importance of grains can be easily illustrated by recalling the accretion time-scale for gas-phase species (eq. [10]). At a density of $10^{4} \mathrm{~cm}^{-3}$ this time-scale is about $10^{5} \mathrm{yr}$, whigh is less than the free-fall time-scale $\left(\tau{ }^{2}{ }^{4} \times 10^{7} /\left[n_{0}\right]^{1 / 2}=4 \times 10^{5}\right.$ yr; Spitzer 1978). Thus, for $n_{0}=10^{4 \mathrm{fm}} \mathrm{cm}^{-3}$, gas-phase molecules accrete much faster on a grain than a cloud collapses to form a star. One might therefore expect that no molecules will be observed at such high densities in molecular clouds (Watson 1976; Greenberg 1979). This is in stark contrast, however, with the observations. Molecules such as $\mathrm{H}_{2} \mathrm{CO}$ and $\mathrm{NH}_{3}$ are routinely observed in dense cloud cores. Moreover, elemental depletions in grain mantles range up to only $40 \%$ and are typically much less ( $T i e l e n s$ et al. 1986a). This suggests that there is an efficient exchange between the gas phase and grain mantles. However, although the accretion time-scale may presently be less than the freefall time-scale, it need not have been in the past. In particular, a newly formed star will raise the temperature of the dust in its neighborhood above the critical temperature for grain-mantle formation ( $=30 \mathrm{~K}$, compare the accretion time-scale of abundant species with their evaporation time-scale; see Table II).

The high abundance of molecular hydrogen in molecular clouds also suggests an important contribution from grain-surface chemistry to the gas-phase composition. There is no efficient gas-phase reaction network for the formation of $\mathrm{H}_{2}$. For that reason molecular hydrogen is generally assumed to be formed on grain surfaces by recombination of two hydrogen atoms or by hydrogen abstraction reactions (see \$5). This assessment is, however, based on negation of other possibilities and there is no direct (chemical) evidence. Although the formation and ejection of $\mathrm{H}_{2}$ on grain surfaces does not necessarily imply that other grain surface reactions contribute to the gas-phase composition, it is suggestive. Certainly, there is good observational evidence for the formation of molecules by grain-surface reactions in the composition of grain mantles (Tielens and Hagen 1982; Tielens et al. 1984; Tielens et al. 1986a).

As argued in \$8, grain-mantle "explosions" are required to explain the observed abundance of the organic refractory dust component in the diffuse interstellar medium. Laboratory grain-mantle analog studies have provided good experimental evidence for an efficient return of grain-mantle molecules to the gas phase upon warm-up of photolyzed mixtures, as well as for the formation of an organic refractory residue (Hagen et al. 1979; d'Hendecourt et al. 1982; d'Hendecourt 1984; 
d'Hendecourt et al. 1986; van de Bult 1986). Simple theoretical arguments, such as those in $\$ 8$ and below, suggest that UV photolysis and transient heating of grains can indeed be of importance.

In this section, the physical processes that may return grainmantle molecules back to the gas phase are discussed. Some observational signatures of grain chemistry will also be indicated. It should be emphasized that there is abundant evidence for the importance of gasphase reactions in the formation of simple gas-phase molecules. In particular, the molecular ions, $\mathrm{HCO}^{+}$and $\mathrm{DCO}^{+}$, provide key support for ion-molecule gas-phase reactions.

\subsection{Ejection of Grain-Mantle Molecules}

If the accretion of gas on grains is offset by ejection of the accreted material, then grains may play an important role in the composition of the gas phase. Model calculations of the gas-phase composition of molecular clouds in which grain-surface reactions were used alone or in conjunction with gas-phase reactions have been performed by several groups (Allen and Robinson 1977; Viala et al. 1979; d'Hendecourt et al. 1985). The contribution of grains to the gas-phase composition in all of these models hinges to a large extent on the identification of ejection mechanisms for molecules formed on the grain surface. Among the ejection mechanisms suggested are thermal evaporation (including singlephoton heating events for very small grains), ejection upon reaction, photodesorption and release of stored chemical energy (cf. Watson 1976; Tielens and Hagen 1982; d'Hendecourt et al. 1982).

The first two processes can be of importance for $\mathrm{H}_{2}$ because of its low binding energy and because of its small mass, which makes energy transfer to the surface relatively inefficient (cf. \$4.6). For other molecules, the efficiency of these ejection processes is expected to be low.

Photodesorption is probably an efficient ejection mechanism for small molecules on grain surfaces in the diffuse interstellar medium or at the edges of molecular clouds. The absorption of a UV photon by a molecule on the surface will electronically excite this molecule. Photodesorption may then occur because of the electronic repulsion between the excited admolecule and neighboring surface molecules. Essentially, the larger orbital distribution of the electronically excited state coupled with the asymmetric force field of the surface will give the nucleus of the admolecule momentum perpendicular to the surface, and ejection may occur. (Nishi et al. 1984). For small molecules the efficiency of this process can be fairly large (Greenberg 1973). Larger molecules will, however, have a relatively low photodesorption efficiency because many more energy-decay channels are available and because they may have a larger binding energy. The 
photodesorption yield will also be low when the excitation energy can be rapidly transferred to other molecules in the particle (e.8., nearresonant transfer with a time-scale of the order of $10^{-13}-10^{-14} \mathrm{sec}$ ). Thus, for an $\mathrm{H}_{2} \mathrm{O}$ molecule on a clean substrate the photodesorption yield is much higher than for $\mathrm{H}_{2} \mathrm{O}$ adsorbed on an $\mathrm{H}_{2} \mathrm{O}$ surface (Greenberg 1973). Using values typical for small molecules and assuming the average interstellar UV radiation field, photodesorption can keep a grain surface clean for $A_{v}$ less than about 3 mag (Tielens and Hagen 1982).

The most important mechanism for ejection of molecules in a grain mantle into the gas phase inside a dense moleçular cloud is probably the release of stored chemical energy. At low temperatures, radicals produced by UV photolysis can be stored in a grain mantle up to a concentration of about $1 \%$ ( $c f . \$ 8$ ). Upon warm-up, these radicals can diffuse and react upon encounter. Laboratory studies on warm-up of photolyzed mixtures show that upon heating to about $27 \mathrm{~K}$, those samples with a poor heat contact with the substrate will explosively eject most of the : mixture (d'Hendecourt et al. 1982; d'Hendecourt 1984). The critical temperature of $27 \mathrm{~K}$ seems to be a general property of irradiated molecular ices, for it is independent of the mixture. Apparently, at this temperature rapid diffusion of most of the trapped radicals occurs simultaneously.

It should be emphasized that these explosions are not a result of a chain reaction $(e .8$. , the reaction heat of a recombining radical driving the diffusion and reaction of other radicals). The deposition of $5 \mathrm{eV}$ of reaction heat in a volume corresponding to 200 molecules (i.e., a radical concentration of $1 \%$ ) raises the temperature to about $170 \mathrm{~K}$ (see below). The temperature relaxation time $T$, owing to heat diffusion, is given by (Anderson 1981) as

$$
\tau_{T}=40 C r_{0}^{2} /\left(\pi^{2} K\right) \text {, }
$$

where $\rho$ is the density; $C$ the specific heat $\left(=2 \times 10^{4} \mathrm{~T}^{1.3} \mathrm{erg} \mathrm{cm}-3 \mathrm{~K}\right.$; Leger et al. 1985); $r_{0}$ the radius of the initial geposition region $(12 \mathrm{~A})$; and $\mathrm{K}$ the thermal conductivity $\left(\approx 3 \times 10^{4}\right.$ erg sec $\mathrm{cm}^{-1} \mathrm{~K}^{-1}$; d'Hendecourt 1984). Inserting these values yields a temperature relaxation time-scale of $3 \times 10^{-12} \mathrm{sec}$. Within that time-scale, however, a radical can migrate at best only a few sites and, given the low concentration of radicals, it will not have found a reaction partner. Thus, the heat liberated by the reaction does not promote reaction of other stored radicals.

It is probable that the existence of a unique critical temperature is a general property of amorphous molecular matrices. At about $25 \mathrm{~K}$ the heat conductivity of an amorphous material changes abruptly in character (Anderson 1981). Below that temperature the energy is carried by low-frequency phonons $\left(\leq 10^{92} \mathrm{sec}^{-1}\right)$ which have a long mean free path 
$\left(\approx 10^{5} \AA\right)$. These phonons interact only with tunneling sites where the amorphous solid can tunnel from one amorphous state to another (cf. \$2). Above that temperature the heat is carried by high-frequency phonons $\left(\approx 10^{12} \mathrm{sec}^{-1}\right)$ with a mean free path of about $10 \AA$. It is these latter, localized phonons that assist in diffusion processes.

We will now estimate the amount of grain-mantle material evaporated by one cosmic ray event or a grain-grain collision. We will assume that enough energy is deposited to raise the grain temperature to the critical temperature $\left(\Delta E \approx 5 \times 10^{-8} \mathrm{erg}\right.$ for a $1000 \AA$ grain at $\left.27 \mathrm{~K}\right)$. For simplicity it is assumed in this calculation that the grain consists mainly of $\mathrm{H}_{2} \mathrm{O}$ with a trace $(1 \%$; $c f$. $\$ 8$ ) of radicals that will start to diffuse and react at this temperature. The reaction energy liberated raises then the temperature enough to cause appreciable evaporation of the mixture. With a $1 \%$ concentration of radicals, assuming a reaction energy of $5 \mathrm{eV}$ and a specific heat of $\mathrm{H}_{2} \mathrm{O}$ ice of about $2 \times 10^{4} \mathrm{~T}^{1.3} \mathrm{erg} \mathrm{cm}-3(\mathrm{~T}>50 \mathrm{~K})$; Leger et al. 1985), this yields a temperature of about $170 \mathrm{~K}$. This is well above the evaporation temperature of $\mathrm{H}_{2} \mathrm{O}$ ice in the interstellar medium ( $90 \mathrm{~K}$; Nakagawa 1980), and evaporation of the mantle will dominate over other heat-loss mechanisms, such as radiation. The amount of material evaporated can then be estimated by equating the reaction heat with the heat loss through evaporation. The energy remaining in the particle at the evaporation temperature should be subtracted since this energy will be carried away by radiation. For the assumptions stated above, this leads to $0.019 \mathrm{eV}$ per $\mathrm{H}_{2} \mathrm{O}$ molecule available for evaporation, which should be compared to the binding energy of $0.05 \mathrm{eV}$. Thus, about $40 \%$ of the $\mathrm{H}_{2} \mathrm{O}$ molecules will evaporate before the temperature will drop below the evaporation temperature. Note that this is less than estimated by Leger et al. (1985), because they assumed that grain mantles consist mainly of $\mathrm{CO}$ with a binding energy of only about $0.09 \mathrm{eV}$. Cosmic ray heating seems to dominate the transient grain heating process with a time-scale of about $3 \times 10^{4}$ yr for a grain size of 1000 Grain-grain collisions are only important at high densities $\left(n_{0} \geq 10^{6} \mathrm{~cm}^{-3}\right)$. With the efficiency calculated above, the ejection time-scale for a molecule is thus about $10^{5} \mathrm{yr}$.

\subsection{Signatures of Grain Chemistry}

Molecules produced by grain-surface chemistry are typically neutral, saturated, small, and highly hydrogenated. Infrared absorption studies of protostars show that $\mathrm{H}_{2} \mathrm{O}$ and alcohols (presumably $\mathrm{CH}_{3} \mathrm{OH}$ ) dominate the composition of interstellar grain mantles; $\mathrm{NH}_{3}$ is also present as a trace (Tielens et al. 1986a). These molecules are the grain-mantle reaction products of the abundant gas-phase species $\mathrm{O}, \mathrm{O}_{2}, \mathrm{CO}$, and $\mathrm{N}$. The measured abundances of $\mathrm{H}_{2} \mathrm{O}$ and $\mathrm{CH}_{3} \mathrm{OH}$ in grain mantles ranges from 
$4 \times 10^{-6}$ to $2 \times 10^{-4}$ per hydrogen atom, which is much larger than their gas-phase abundances. Obviously, these molecules, which are easily made on grain surfaces, would be prime candidates for studies of the influence of grains on the gas-phase composition. In this respect, it should be noted that species produced by gas-phase chemistry are often highly unsaturated, radicals, or ions.

In general, the relative contribution of grains to the composition of the gas phase is expected to increase with density. Gas-phase chemistry is driven by ion-molecule reactions. The degree of ionization decreases, however, with increasing density, essentially because recombination increases faster with increasing density that ionization (Oppenheimer and Dalgarno 1974; Prasad and Huntress 1980). Thus, the rate of ion-molecule reactions is expected to increase slower with density than the rate of grain-surface reactions. The rate of production of species on grain surfaces is limited by the accretion rate of the appropriate reactants onto grains. At molecular cloud densities, this means that only for those molecules that are easily made on a grain surface but are slowly produced in the gas phase, grains will contribute to the gas-phase abundance.

Thus, one can test the importance of grain chemistry for the composition of the gas phase by searching for gas-phase molecules in molecular clouds that are difficult to form by ion-molecule reaction schemes. Obvious candidates are molecular species containing metal atoms (e.8., NaH or $\mathrm{NaOH})$. These species are not expected to form molecular species through gas-phase reactions ( $c f$. Oppenheimer and Dalgarno 1974; Smith et al. 1983). However, these species will react on grain surfaces.

One might expect, naively, that the overabundance of atomic hydrogen will convert these species into hydrides, as observations show has happened to oxygen, nitrogen, and carbon monoxide; however, the grainsurface chemistry of such species is somewhat more complicated. In particular, hydrogen abstraction reactions can be of importance (-Barlow 1978). For example, the reaction $\mathrm{HaH}+\mathrm{H} \rightarrow \mathrm{Na}+\mathrm{H}_{2}$ is exothermic by about $2.5 \mathrm{eV}$. Because a molecular bond has to be broken in order for this reaction to proceed, there will be an activation barrier that has to be overcome. Typically, such an activation barrier is about $5 \%$ of the bond energy $(\approx 1000 \mathrm{~K})$, which is of little consequence on grain surfaces. Thus, in effect, $\mathrm{Na}$ like $\mathrm{N}_{2} \mathrm{H}_{4}, \mathrm{H}_{2} \mathrm{CO}$, and other molecules will act as chemical bonding sites for $\mathrm{H}_{2}$ formation (cf. \$5). Hydrogen addition and abstraction reactions will continue to form $\mathrm{H}_{2}$ with $\mathrm{Na}$, until it reacts with another migrating species such as 0 , which effectively poisons the site for $\mathrm{H}_{2}$ formation. Since the energy barrier is expected to be low and because atomic oxygen is the next most abundant migrating radical on a grain surface, most sodium atoms are expected to wind up in $\mathrm{NaOH}$ molecules. Hydrogen abstraction from these molecules is 
presumably inhibited by a high actuation energy. It is not yet possible to search for the vibrational transitions of molecules with such low abundances in interstellar grain mantles, but it is possible to search for the rotational transitions of the gas-phase species. Nitrogen and sulfur bearing, gas-phase molecules (e.g., $\mathrm{NH}_{3}$ and $\mathrm{H}_{2} \mathrm{~S}$ ) seem difficult to form by ion-molecule reactions (Prasad and Huntress 1980, 1982). This would also make them prime candidates for studies of the importance of grains for the gas-phase composition of molecular clouds. Both $\mathrm{NH}_{3}$ and $\mathrm{H}_{2} \mathrm{~S}$ have tentatively been identified in grain mantles (Knacke et al. 1982; Geballe et al. 1985). However, the gas-phase chemistry of these compounds is still open to some debate (cf. Graedel et al. 1982), which somewhat limits their use as grain-chemistry indicators. Complex molecules seem also to be difficult to make by gasphase reactions. Grain-surface reactions will not be much help because they generally lead to fairly simple, completely hydrogenated molecules. Ultraviolet photolysis of grain mantles will, however, convert such molecules into more complex ones which may eventually be injected into the gas phase. The importance of this process is difficult to assess. The first step seems to be to identify such molecules in interstellar grain mantles.

As discussed before, grain-surface chemistry can lead to large deuteration effects (cf. \$7 and Fig. 14). Essentially, this reflects the high gas-phase ratio of atomic $D$ to $H$. This ratio is high because of the rapid destruction of $\mathrm{HD}$ through reactions with $\mathrm{H}_{3}^{+}$(Tielens 1983b; cf. \$7). Generally, high density and low gas-phase temperatures lead to high abundances of deuterated molecules with respect to their hydrogenated counterparts. The temperature dependence arises from the temperature sensitivity of the gas-phase $\mathrm{H}_{2} \mathrm{D}^{+}$plus $\mathrm{H}_{2}$ reactions. Prime candidates for such studies are $\mathrm{HDO}, \mathrm{NH}_{2} \mathrm{D}, \mathrm{DNO}, \mathrm{HDCO}$, and possibly $\mathrm{CH}_{2} \mathrm{DOH}$. Studies of the deuteration of ammonia may be particularly revealing, since the ratios $N_{3-i} D_{i} / N_{2-i} D_{i+1}(i=0,1,2)$ should all be equal to the gas-phase $n(H) / n(D)$ ratio, which at low temperatures is just $A(D) n_{Q}$, where $A(D)$ is the elemental abundance of deuterium.

Einally, the ortho/para ratio can serve as a grain-chemistry indicator. Some molecules exist in two distinct states, depending on the spin of the nucleus: the para state when the spins are anti-parallel and the ortho state when they are parallel. Because of the symmetry of the wave function, the para states are associated with the even rotational levels and the ortho states with the odd levels. Rotational transitions between ortho and para states require a simultaneous transition of the nuclear spins of the molecule. The radiative lifetime for decay from the ortho to the para state is therefore long. The energy difference between these two states can be quite large compared to interstellar temperatures (i.e., for $\mathrm{H}_{2}$ about $170 \mathrm{~K}$ and for $\mathrm{H}_{2} \mathrm{O}$ about $55 \mathrm{~K})$, and small variations in temperature can thus have large effects. 
The initial ortho-para ratio of molecules such as $\mathrm{H}_{2} \mathrm{O}, \mathrm{NH}_{3}, \mathrm{H}_{2} \mathrm{CO}$, and $\mathrm{CH}_{4}$, newly formed on a grain surface, is expected to be equal to the high-temperature limit (i.e., the ratio of the statistical weights of the two nuclear spin states). This is due to the large reaction heat available upon formation and the considerable rearrangement of the hydrogen nuclei involved in most reactions. Ortho-para transformation will occur on a time-scale of a day because of the interaction of the nuclear spins of neighboring molecules. An even faster ortho-para transformation time-scale may result from the presence of paramagnetic impurities or lattice defects (Tielens 1986b). The nuclear spin transition is due to the inhomogeneous magnetic dipole field generated by the nuclear spin of a neighboring molecule or the unpaired electron of a paramagnetic impurity or lattice defect. This couples to the proton magnetic moments in such a way as to cause a transition between the or tho and para states (Wigner 1933). Since the residence time of molecules produced on grain surfaces is expected to be about $10^{5} \mathrm{yr}$, their ortho-para ratio will reflect the dust temperature (i.e., $n(0) / n(p)=g \exp \left(-E_{o p} / T_{d}\right)$, where $g$ is the ratio of the statistical weights of the nuclear spin states, $E_{o p}$ the ortho-para energy difference, and $T_{d}$ the dust temperature). Because ejection into the gas phase is a rapid process, it will not influence this ratio.

It should be emphasized that once the molecules are in the gas phase, ion-molecule reactions may influence the ortho-para ratio. Probably the most important reaction in this respect is $\mathrm{H}$ exchange with $\mathrm{H}_{3}^{+}$. Assuming a Langevin reaction rate coefficient $\left(10^{-9} \mathrm{~cm}^{3} \mathrm{sec}^{-1}\right)$ and $a^{3}$ relative abundance of $\mathrm{H}_{3}^{+}$of $10^{-8}$ (Smith and Adams 1984; Lepp et al. 1986) yields a time-scale for this process of $3 \times 10^{9} / \mathrm{n}_{\mathrm{ogr}} \mathrm{yr}$. This is comparable to the time-scale for accretion on grains $\left(109 / \mathrm{n}_{0} \mathrm{yr}\right)$, which is probably the limiting step in the grain-surface reactions.

From this discussion, it is clear that in a region where the dust and gas temperature are sufficiently different relative to the orthopara energy difference, the ortho-para ratio can be used as a discriminant for grain-gas interaction (Tielens 1986b). It should be emphasized that the ortho-para transformation process discussed above is not a chemical mechanism; strictly speaking, the ortho-para ratio will therefore not prove the importance of grain chemistry for the composition of the gas phase. However, the importance of grain chemistry for the composition of grain mantles is already well attested to by IR spectroscopy.

The best objects in which to study the ortho-para ratio are cold, dense, dark clouds such as Taurus. One such study (for $\mathrm{H}_{2} \mathrm{CO}$ ) has been performed for two dark clouds (and the warm gas in Orion; Kahane et al. 1984). Since the energy difference between the ortho and para states of $\mathrm{H}_{2} \mathrm{CO}$ is small $(15 \mathrm{~K})$ and the lines suffer from beam dilution, the interpretation is not straightforward; nevertheless it seems that in dark 
clouds thermalization on grains dominates, implying an efficient gasgrain exchange mechanism.

\section{ACKNOWLEDGMENT}

We thank Dave Hollenbach for many stimulating discussions and a critical reading of an earlier version of this manuscript.

\section{REFERENCES}

Aitken, D. K. 1981, in IAU Symp. No. 96, Infrared Astronomy, eds. G. C. Wynn Williams and D. P. Cruikshank (Reidel, Dordrecht), p. 207.

Aitken, D. K., and Roche, P. F. 1984, M. N. R. A. S., 208, 751.

Allamandola, L. J., Tielens, A. G. G. M., and Barker, J. R. 1985, AP. J. Letters, 290, L25.

Allamandola, L. J., Tielens, A. G. G. M., and Barker, J. R. 1986, in preparation.

Allen, D. A. and Wickramasinghe, D. T. 1981, Nature, 294, 239.

Allen, M., and Robinson, G. W. 1977, Ap. J., 212, 396.

Anderson, H. L. 1981, Physics Vademecum (American Institute of Physics, New York).

Aronowitz, S., and Chang, S. 1985, Ap. J., 293, 243.

Balooch, M., and Olander, D. R. $1975 \mathrm{~J}$. Chem. Phys., 63, 4772.

Barlow, M. J. 1978, M. N. R. A. S., 183, 397.

Bass, A. M., and Broida, H. P. 1966, Formation and Trapping of Free Radicals (Academic Press, New York).

Beckwith, S., Persson, S. E., Neugebauer, G., and Becklin, E. E. 1978, Ap. J., 223, 464.

Bedijn, P.. 1977, Ph.D. thesis, Leiden.

Bell, R. J., and Dean, P. 1972, Phil. Mag., 25, 1381.

Bohlin, R. C., Savage, B. D., and Drake, J. F. 1978, Ap. J., 224, 132.

Bohren, C. F., and Huffman, D. R. 1984, Absorption and Scattering of Light by Small Particles (Wiley and Sons, New York).

Bondybey, V. E., and Brus, L. E. 1980, Adv. Chem. Phys., 41, 269.

Borghesi, A., Bussoletti, E., and Colangeli, L. 1983, Astr. Ap., 142, 225.

Brus, L. E. 1981, J. Chem. Phys., 74, 737.

Burke, J. R., and Hollenbach, D. J. 1984, Ap. J., 265, 223.

Butchart, I., McFadzean, A. D., Whittet, D. C. B., Geballe, T., and Greenberg, J. M. 1985, Astr. Ap., 154, L5.

Calvert, J. G., and Pitts, J. N. 1966, Photochemistry (Wiley, New York).

Campbell, M. F., et al. 1976, Ap. J., 208, 396. 
Cohen, M., Allamandola, L. J., Tlelens, A. G. G. M., Bregman, J., Simpson, J. P., Witteborn, F. C., Wooden, D., and Rank, D. 1986, Ap. J., 302, 737.

d'Hendecourt, D. B., Allamandola, L. J., and Greenberg, J. M. 1985. Astr. Ap., 152, 130.

d'Hendecourt, L. B. 1984, Ph. D. thesis, Leiden.

d'Hendecourt, L. B., Allamandola, L. J., Baas, F., and Greenberg, J. M. 1982, Astr. Ap., 109, L12.

d'Hendecourt, L. B., Allamandola, L. J., Grim, R. J. A., and Greenberg, J. M. 1986, Astr. Ap., 158, 119.

Dalgarno, A. 1975, in Atomic and Molecular Processes in Astrophysics, eds. M. C. E. Huber and H. Nussbaumer, (Geneva Observatory, Geneva), p. 1.

Day, K. L. 1976, Ap. J., 210, 614.

Day, K. L. 1979, Ap. J., 234, 158.

Day, K. L., and Donn, B. 1978, Ap. J. Letters, 222, 445.

de Boer, J. H. 1968, The Dynamical Character of Absorption, (Clarendon Press, Oxford).

de Boer, J. H., and van Steenis, J. 1952, Proc. Kon. Ned. Akad. Wet., B55, 572.

Diestler, D. J. 1974, J. Chem. Phys.., 60, 2692.

Diestler, D. J., Knapp, E-W., and Ladouceur, H. D. 1977, J. Chem, Phys., 68, 4056 .

Donn, B. 1960, in Formation and Trapping of Free Radicals, eds. A. M. Bass, and H. P. Broida (Academic Press, New York), p. 347.

Donn, D., and Jackson, W. M. 1970, B. A. A. S., 2, 309.

Draine, B. T., and Lee, H. M. 1984, Ap. J., 285, 89.

Draine, B. T. 1979, Ap. J., 230, 106.

Draine, B. T. 1984, Ap. J. Letters, 277, 271.

Draine, B. T., and Salpeter, E. E. 1979, Ap. J., 231, 438.

Duley, W. W., and McCullough, J. D. 1977, Ap. J. Letters, 211, L145.

Duley, W. W., and Williams, D. A. 1981, M. N.R..A. S., 196, 269.

Ehrenreich, H. 1972, Eundamentals of Amorphous Semi Conductors,

(National Academy of Sciences, New York).

Einstein, T. L., Hertz, J. A., and Schieffer, J. R. 1980, in Theory of

Chemisorption, ed. J. R. Smith (Springer Verlag, Berlin), p. 183.

Fegley, B., and Lewis, J. S. 1980, Icarus, $41,439$.

Field, G. B. 1974, Ap. J., 187, 453.

Flannery, B. P., Roberge, W., and Rybicki, G. B. 1980, Ap. J., 236 , 598.

Frenkel, J. 1924, 2. Physik, 26, 117.

Gatley, I., Becklin, E. E., Werner, M. W., and Wynn Williams, C. G. 1977, Ap. J., 216, 277.

Gatley, I., Garden, R., Brand, P. W. J. L., Lightfoot, J., Glencross, W., Okuda, H., and Nagata, T. 1986, in preparation. 
Geballe, T. R., Baas, F., Greenberg, J. M., and Schutte, W. 1985, Astr. Ap., 146, L6.

Gelb, A., and Kim, S. K. 1971, J. Chem. Phys., 55, 4935.

Gillett, F. C., Jones, T. W., Merrill, K. M., and Stein, W. A. 1975a, Astr. Ap., 45, 77.

Gillett, F. C., Forrest, W. J., Merrill, K. M., Capps, R. W., and Soifer, B. T. 1975b, Ap. J., 200, 609.

Gilra, D. P. 1972, in The Scientific Results from the Orbiting Astronomical Observatory OAO-2, ed. A. D. Code, NASA SP-310, p. 297.

Gould, R. K. 1975, J. Chem. Phys., 63, 1825.

Govers, T. R., Mattera, L. and Scoles, G. 1980, J. Chem. Phys., 72, 5446.

Graedel, T. E., Langer, W. D., and Frerking, M. A. 1982, Ap. J. Suppl., $48,321$.

Greenberg, J. M., and Hong, S. S. 1974, in I. A. U. Symp. No. 60, Galactic Radio Astronomy, eds. F. J. Kerr and S. C. Simonson (Reidel, Dordrecht), p. 155.

Greenberg, J. M. 1982, in Submillimetre Wave Astronomy, eds. J. E. Beckman and J. P. Phillips (Cambridge University Press, Cambridge), p. 261.

Greenber8, J. M. 1963, Ann. Rev. Astr. Ap., 15, 267.

Greenberg, J. M. 1979, in Stars and Stellar Systems, ed. B. Westerlund (Reidel, Dordrecht), p. 173.

Greenberg, J. M., and Chlewicki, G. 1984, Ap. J., 272, 563.

Greenberg, J. M., and Yencha, A. J. 1973, in I. A. U. Symp. No. 52, Interstellar Dust and Related Topics, eds. J. M. Greenberg and $H$. C. van de Hulst (Reidel, Dordrecht), p. 309.

Greenberg, L. T. 1973, in I. A. U. Symp. No. 52, Interstellar Dust and Related Topics, eds. J. M. Greenberg and H. C. van de Hulst (Reidel, Dordrecht), p. 413.

Habing, H. J. 1968, Bull. Astron. Inst. Neth., 19, 421. Hackwell, J.A. 1971, Ph. D. thesis, University College, London.

Hadni, A. 1970, in Ear-Infrared Properties of Solids, eds. S. S. Mitra and S. Nudelman (Plenum Press, New York), p. 561.

Hagen, W. 1982, Ph, D. thesis, Leiden.

Hagen, W., Tielens, A. G. G. M., and Greenberg, J. M. 1981, Chem. Phys., 56, 367 .

Hagen, W., Tielens, A. G. G. M., and Greenberg, J. M. 1983a, Astr. Ap.Suppl., 51, 389.

Hagen, W., Tielens, A. G. G. M., and Greenberg, J. M. 1983b, Astr. Ap., $117,132$.

Hagen, W., Allamandola, L. J., and Greenberg, J. M. 1979, Astr. Space Sci., $65,215$. 
Hagen, W., Allamandola, L. J., and Greenberg, J. M. 1980, Astr. Ap., 86, L3.

Hair, M. L. 1967, Infrared Spectroscopy in Surface Chemistry (Dekker, New York).

Harvey, P. M., Hoffmann, W. F., and Campbell, M. F. 1978, Astr. Ap., 70,165 .

Hasegawa, H., and Koike, C. 1984, in Laboratory and Observational Infrared Spectra of Interstellar Dust, eds. R. D. Wolstencroft and J. M. Greenberg (Oc. Rep. Royal Obs. Edinburgh), p. 137.

Hickmott, T. W. 1960, J. Appl. Phys., 31, 128.

Hollenbach, D., and Salpeter, E. E. 1970, J. Chem. Phys., 53, 79.

Hollenbach, D., and Salpeter, E. E. 1971, Ap. J., 163, 155.

Hoyle, F., and Wickramasinghe, N. C. 1962, M. N. R. A. S., 124, 417.

Hunter, D. A., and Watson, W. D. 1978, Ap. J., 226, 477.

Jaycock, M. J., and Parfitt, G. D. 1986, Chemistry of Interfaces (Wiley and Sons, New York).

Jones, T. W., and Merrill, K. M. 1976, Ap. J., 209, 509.

Joyce, R. R. and Simon, T. 1982, Ap. J., 260, 604.

Kahane, C., Frerking, M. A., Langer, W. D., Encrenaz, P., and Lucas, R. 1984, Astr. Ap., 137, 211.

Kameijo, F. 1963, Publ. Astr. Soc. Japan, 15, 440.

Khare, B. N., and Sagan, C. 1973, in Molecules in the Galactic Environment, eds. M. A. Gordon, and L. E. Snyder (Wiley and Sons, New York), p. 399.

King, A. B., and Wise, H. 1963, J. Phys. Chem., 67, 1163.

Kittell, C. 1976, Introduction to Solid State Physics (Wiley and Sons, New York).

Knacker, R. F., McCorkle, S., Puetter, R. C., Erickson, E. F., and Kratschmer, W. 1982, Ap. J., 260141.

Koike, C., Hasegawa, H., and Manabe, A. 1980, Astr. Space Sci., 67, 495.

Lacy, J. H., Baas, F., Allamandola, L. J., Persson, S. E., McGregor, P. J., Lonsdale, C. J., Geballe, T. R., and van de Bult, C. E. P. 1984, Ap. J., 276, 533.

Landau, L. D., and Lifshitz, E. M. 1960, Course of Theoretical Physics, Vol. 1, Mechanics (Pergamon Press, New York).

Langmuir, I. 1912, J. Am. Chem. Soc., 34, 1310.

Langmuir, I. 1915, J. Am. Chem. Soc., 37, 417.

Lee, T. J. 1975, Astr. Space Sci., 34, 123.

Legay, F. 1977, in Chemical and Blochemical Applications of Lasers,

vol. 2, ed. C. B. Moore (Academic Press, New York), p. 1.

Leger, A., and Puget, J. L. 1984, Astr. Ap., 137, L5.

Leger, A., Jura, M., and Omont, A. 1985, Astr. Ap., 144, 147.

Leitch-Devlin, M. A., and Williams, D. A. 1985, M. N. R. A. S., 213, 295. 
Lepp, S., Dalgarno, A., and Sternberg, A. 1986, preprint. Lillie, C. F., and Witt, A. N. 1976, Ap. J., 208, 64.

Lucas, D., and Ewing, G. E. 1981 , Chem. Phys., 58, 385.

Madden, W. G., Bergren, M.S., McGraw, W. R., and Rice, S. A. 1978, J. Chem. Phys., 69, 3497.

Mathis, J. S., and Wallenhorst, S. G. 1981, Ap. J., 244, 483.

Mathis, J. S., Rumpl, W. and Nordsieck, K. H. 1977, Ap. J., 217, 425.

Mathis, J. S. 1986, in The Interrelation of Interstellar, Circumstellar and Planetary Dust, eds. J. Nuth and R. Stencel, NASA CP 2403, p. 29.

McCarroll, B., and McKee, D.W. 1971, Carbon, 9, 301.

Merrill, K. M. 1977, in The Interaction of Variable Stars with their

Environments, eds. R. Kippenhahn, J. Rahe, and W. Strohmeier

(Veroff, Remeis Sternwarte, Bamberg), Bd. XI, nr 121, p. 446.

Merrill, K. M., Russell, R. W., and Soifer, B. T. 1976, Ap. J., 207 , 763.

Mitchel1, G. F., Ginsburg, J. L., and Kuntz, P. J. 1978, Ap. J.,

Suppl., 38, 39.

Mooney, T., and Knacke, R. E. 1986, preprint.

Moore, M. H., and Donn, B. 1982, Ap. J. Letters, 257, 447.

Morton, D. C. 1975, Ap. J., 197, 85.

Mueller, W. M., Blackledge, J. P., and Libowitz, G. G. 1968, Metal Hydrides (Academic Press, New York).

Nakagawa, N. 1980, in I. A. U. Symp. No. 87, Interstellar Molecules, ed. B. H. Andrew (Reidel, Dordrecht), p. 365 .

Nishi, N., Shinohara, H., and Okuyama, T. 1984, J. Chem. Phys., 80, 3898 .

Nitzan, A., Mukamel, S., and Jortner, J. 1975, J. Chem. Phys., 63, 200. Norman, C. A., and Silk, J. 1980, Ap.J., 238, 158.

Nuth, J. A., and Donn, B. 1982, J. Chem. Phys., 777, 2639.

Okabe, H.. 1978, Photochemistry of Small Molecules (Wiley and Sons, New York).

Oppenheimer, M., and Dalgarno, A. 1974, Ap.J., 192, 29.

Pauling, L. 1964, College Chemistry (Freeman and Co., San Francisco).

Penman, J. M. 1976, M. N. R. A. S., 175, 149.

Phillips, W. A. 1981, Amorphous Solids, Low Temperature Properties,

(Springer Verlag, New York).

Plendl, J. N. 1970, in Far-Infrared Properties of Solids, eds. S. S.

Mitra and S. Nudelman (Plenum Press, New York).

Prasad, S. S., and Huntress, W. T. 1980, Ap. J. Suppl., 43, 1.

Prasad, S. S., and Huntress, W. T. 1982, Ap. J., 260, 590.

Prasad, S. S., and Tarafdar, S. P. 1983, Ap. J., 267, 603.

Purcel1, E. M. 1969, Ap. J., 158, 433.

Puri, B. R. 1976, in Chemistry and Physics of Carbon, 6, ed. P. L. Walker (Marcel Dekker, New York), p. 191. 
Robeli, A. J., Ballou, E. V., and Boudart, M. 1964, J. Phys. Chem., 68, 2748.

Roche, P. F., and Aitken, D. K. 1984, M. N. R. A. S., 208, 481. Roche, P. E., and Aitken, D. K. 1985, M. N.R.A.S., 215, 425.

Russell, R. W., Soifer, B. T. and Willner, S. P. 1977, Ap. J.Letters, 217, L149.

Rye, R. R. 1977, Surface Sci., 69, 653.

Savage, B. D., and Mathis, J. S. 1979, Ann. Rev. Astr. Ap., 17, 73.

Schutte, W., and Tielens, A. G. G. M. 1985, in Mass Loss from Red

Giants, eds. M. Morris, and B. Zuckerman (Reidel, Dordrecht),

p. 87.

Seki, J., and Yamamoto, T. 1980, Astr. Space Sci., 72, 79.

Sellgren, K. 1986, Ap. J., 305, 399.

Sellgren, K., Werner, M. W., and Dinerstein, H. L. 1983, Ap. J., 271, L13.

Smith, D., and Adams, N. G. 1984, Ap. J. Letters, 284, L13.

Smith, D., Adams, N. G., Alge, E., and Herbst, E. 1983, Ap. J., 272 , 365.

Snoey ink, V. L., and Weber, W. J. 1972, Prog. Surf. Membrane Sci., 5, 63.

Snow, T. P. 1975, Ap. J. Letters, 202, L87.

Spitzer, L. 1976, Comments on Astr., 6, 177.

Spitzer, L. 1978, Physical Processes in the Interstellar Medium (Wiley

and Sons, New York).

Spitzer, L., and Cochran, W. D. 1973, Ap. J. Letters, 186, L23.

Strazulla, G., Calgagno, L. and Foti, G. 1983, M. N.R. A. S., 204, 598 .

Tauc, J. 1972, in Optical Properties of Solids, ed. F. Abeles (North Holland Publishing Company, Amsterdam), p. 278.

Tielens, A. G. G. M. 1983a, Ap. J., 271, 702.

Tielens, A. G. G. M. 1983b, Astr. Ap., 119, 177.

Tielens, A. G. G. M. 1986a; in preparation.

Tielens, A. G. G. M. 1986b, in preparation.

Tielens, A. G. G. M., and deJong, T. 1979, Astr. Ap., 75, 326.

Tielens, A. G. G. M., and Hagen, W. 1982, Astr. Ap., 114, 245.

Tielens, A. G. G. M., Allamandola, L. J., Bregman, J., Goebel, J., d'Hendecourt, L. B., and Witteborn, E. C. 1984, Ap. J., 287, 697.

Tielens, A. G. G. M., Allamandola, L. J., Bregman, J., Witteborn, F. C., Wooden, D., and Rank, D. 1986a, in preparation.

Tielens, A. G. G. M., Allanandola, L. J., Bregman, J., and Witteborn, F. C. 1986b, in preparation:

Touloukian, Y. S., and Buyco, E. H. 1970, Thermophysical Properties of Matter, 5 (Plenum Press, New York).

van de Bult, C. E. P. 1986, private communication.

Veprek, S., and Haque, M. R. 19̣75, Appl. Phys., 8, 303. 
Viala, Y. P., Bel, N., and Clavel, J. 1979, Astr. Ap., 73, 174. Watson, W. D. 1976, in Atomic and Molecular Physics and the Interstellar Matter, eds. R. Balian, P. Encrenaz, and J. Lequeuz (Elsevier, Amsterdam), p. 177.

Watson, W. D., and Salpeter, E. E. 1972, Ap. J., 174, 321.

Wigner, E. 1933, Z. Physik. Chem. B., 23, 28.

Willner, S. P., et al. 1982, Ap. J., 253, 174.

Willner, S. P., Russell, R. W., Puetter, R. C., Soifer, B. T., and Harvey, P. M. 1979, Ap. J. Letters, 229, L65.

Wood, B. J., and Wise, H. 1962, J. Phys. Chem., 66, 1049.

Wood, B. J., and Wise, H. 1969, J. Phys. Chem., 73, 1368.

Wooten, B. J. 1972, Optical Properties of Solids (Academic Press, New York).

Zachariasen, W. H. 1932, J. Am. Chem. Soc., 54, 3841.

Zaikowski, A., Knacke, R. F., and Porco, C. C. 1975, Astr. Space Sci., $35,97$.

Zallen, R. 1983, The Physics of Amorphous Solids (Wiley and Sons, New York). 


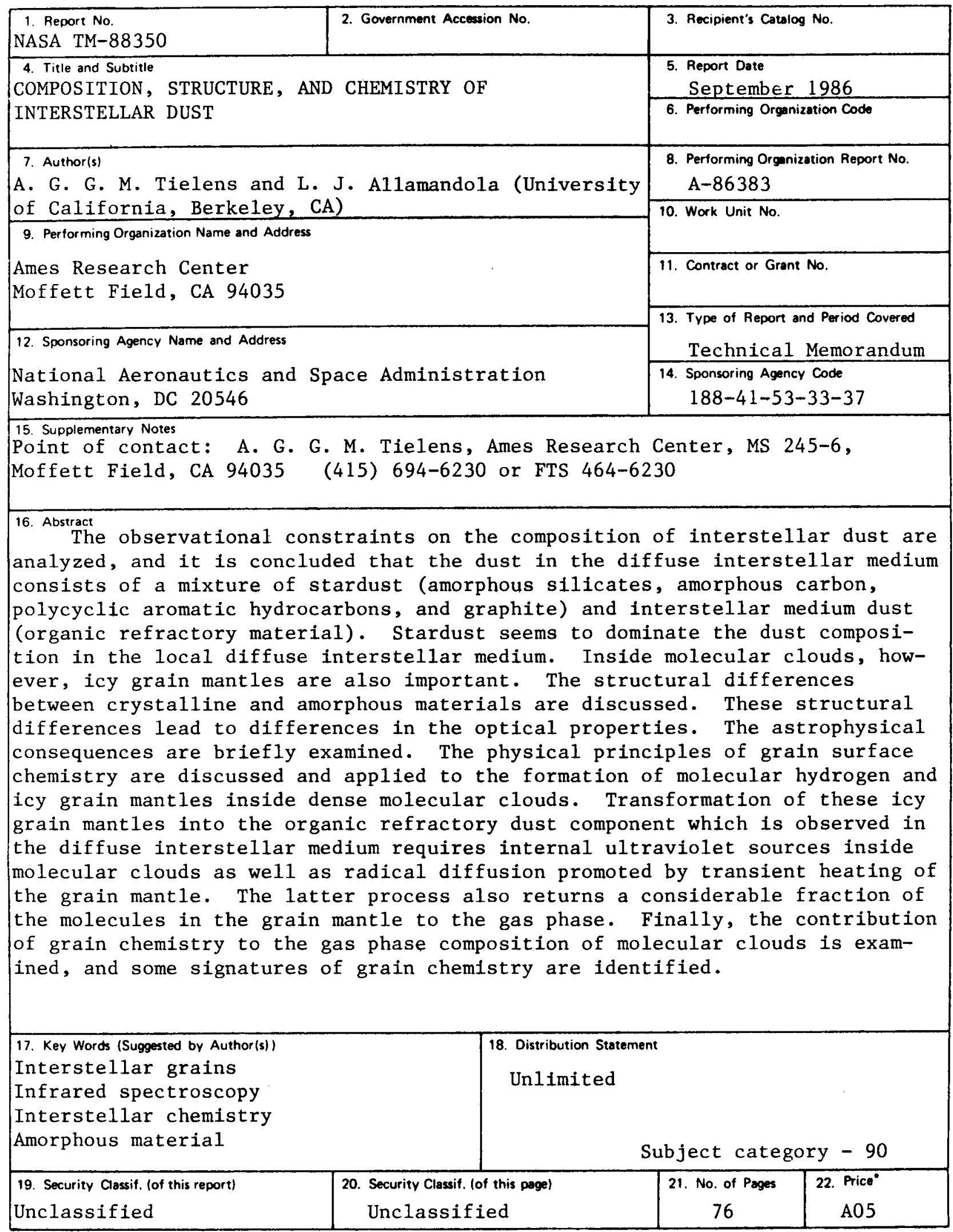

"For sale by the National Technical Information Service, Springfield, Virginia 22161 